\title{
Stability of parabolic Harnack inequalities for symmetric non-local Dirichlet forms
}

\author{
Zhen-Qing Chen*, Takashi Kumagai ${ }^{\dagger}$ and Jian Wang ${ }^{\ddagger}$ \\ In memory of Kazumasa Kuwada.
}

\begin{abstract}
In this paper, we establish stability of parabolic Harnack inequalities for symmetric non-local Dirichlet forms on metric measure spaces under general volume doubling condition. We obtain their stable equivalent characterizations in terms of the jumping kernels, variants of cutoff Sobolev inequalities, and Poincaré inequalities. In particular, we establish the connection between parabolic Harnack inequalities and two-sided heat kernel estimates, as well as with the Hölder regularity of parabolic functions for symmetric non-local Dirichlet forms.
\end{abstract}

AMS 2010 Mathematics Subject Classification: Primary 35B51, 35B35; Secondary 35B65, 28A80, 60J75.

Keywords and phrases: non-local Dirichlet form, parabolic Harnack inequality, Hölder regularity, stability

\section{Introduction and Main Results}

Harnack inequalities are inequalities that control the growth of non-negative harmonic functions and caloric functions (solutions of heat equations) on domains. The inequalities were first proved for harmonic functions for Laplacian in the plane by Carl Gustav Axel von Harnack, and later became fundamental in the theory of harmonic analysis, partial differential equations and probability. One of the most significant implications of the inequalities is that

\footnotetext{
* Research partially supported by Simons Foundation grant 520542, a Victor Klee Faculty Fellowship at UW, and NNSFC 11731009.

${ }^{\dagger}$ Research partially supported by the Grant-in-Aid for Scientific Research (A) 25247007 and 17 H01093.

${ }^{\ddagger}$ Research partially supported by the National Natural Science Foundation of China (No. 11831014), the Program for Probability and Statistics: Theory and Application (No. IRTL1704), the Program for Innovative Research Team in Science and Technology in Fujian Province University (IRTSTFJ), and the JSPS postdoctoral fellowship (26·04021).
} 
(at least for the cases of local operators/diffusions) they imply Hölder continuity of harmonic/caloric functions. We refer readers to [K1] for the history and the basic introduction of Harnack inequalities.

Because of their fundamental importance, there has been a long history of research on Harnack inequalities. Harnack inequalities and Hölder regularities for harmonic functions are important components of the celebrated De Giorgi-Nash-Moser theory in harmonic analysis and partial differential equations. In early 90's, equivalent characterizations for parabolic Harnack inequalities (that is, Harnack inequalities for caloric functions) were obtained by Grigor'yan [Gr] and Saloff-Coste [Sa1] for Brownian motions (or equivalently, Laplace-Beltrami operators) on complete Riemannian manifolds. They showed that parabolic Harnack inequalities are equivalent to doubling condition of the volume measures plus Poincaré inequalities, which are also equivalent to the two-sided Gaussian-type heat kernel estimates. An important consequence of this equivalence is that the parabolic Harnack inequalities are stable under transformations of the Riemannian manifolds by quasi-isometry. This result was later extended to symmetric diffusions on metric measure spaces by Sturm [St] and to random walks on graphs by Delmotte [De]. It has been further extended to symmetric anomalous diffusions on metric measure spaces including fractals in [BBK1.

In this paper, we consider the stability of parabolic Harnack inequalities for symmetric non-local Dirichlet forms (or equivalent, symmetric jump processes) on metric measure spaces. Let $(M, d, \mu)$ be a metric measure space where $d$ is a metric and $\mu$ is a Radon measure (see Section 1.1 for a precise setting). We consider a symmetric regular Dirichlet form $(\mathcal{E}, \mathcal{F})$ on $L^{2}(M ; \mu)$ of pure jump type; that is,

$$
\mathcal{E}(f, g)=\int_{M \times M \backslash \operatorname{diag}}(f(x)-f(y))(g(x)-g(y)) J(d x, d y), \quad f, g \in \mathcal{F},
$$

where diag denotes the diagonal set $\{(x, x): x \in M\}$ and $J(\cdot, \cdot)$ is a symmetric jumping measure on $M \times M \backslash$ diag. Let $X$ be Hunt process corresponding to $(\mathcal{E}, \mathcal{F})$. An important example of the jumping kernel $J$ is $J(d x, d y)=\frac{c(x, y)}{d(x, y)^{d+\alpha}} \mu(d x) \mu(d y)$, where $c(x, y)$ is a symmetric function bounded between two positive constants and $\alpha>0$. The corresponding process is called a symmetric $\alpha$-stable-like process. When $M=\mathbb{R}^{d}$, or more general, an Ahlfors $d$-regular space, $\mu$ is the Hausdorff measure on $M$ and $\alpha \in(0,2)$, various properties of the symmetric $\alpha$-stable-like processes including two-sided heat kernel estimates and parabolic Harnack inequalities have been studied in CK1. In particular, when $M=\mathbb{R}^{d}, \mu$ is the Lebesgue measure on $\mathbb{R}^{d}$ and $c(x, y)$ is a constant function, this corresponds simply to a rotationally symmetric $\alpha$-stable Lévy process. However, on some metric measure spaces $M$ such as the Sierpinski gasket and the Sierpinski carpet, the index $\alpha$ can be larger than 2; see Example 5.1.

Let $\phi$ be a strictly increasing continuous function on $[0, \infty)$ with $\phi(0)=0$.

Definition 1.1. We say that the parabolic Harnack inequality $\operatorname{PHI}(\phi)$ holds for the process $X$, if there exist constants $0<C_{1}<C_{2}<C_{3}<C_{4}, 0<C_{5}<1$ and $C_{6}>0$ such that for every $x_{0} \in M, t_{0} \geq 0, R>0$ and for every non-negative function $u=u(t, x)$ on $[0, \infty) \times M$ that is caloric (or space-time harmonic) in cylinder $Q\left(t_{0}, x_{0}, C_{4} \phi(R), R\right):=$ 
$\left(t_{0}, t_{0}+C_{4} \phi(R)\right) \times B\left(x_{0}, R\right)$,

$$
\operatorname{ess} \sup _{Q_{-}} u \leq C_{6} \operatorname{ess}_{\inf _{Q_{+}} u}
$$

where $Q_{-}:=\left(t_{0}+C_{1} \phi(R), t_{0}+C_{2} \phi(R)\right) \times B\left(x_{0}, C_{5} R\right)$ and $Q_{+}:=\left(t_{0}+C_{3} \phi(R), t_{0}+C_{4} \phi(R)\right) \times$ $B\left(x_{0}, C_{5} R\right)$.

We call the function $\phi$ the scale function for $\operatorname{PHI}(\phi)$. The $\operatorname{PHI}(\phi)$ results obtained in Gr, Sa2, St, De are for $\phi(r)=r^{2}$. It is proved in CK1 that symmetric $\alpha$-stable-like processes with $\alpha \in(0,2)$ enjoy $\operatorname{PHI}(\phi)$ for $\phi(r)=r^{\alpha}$. In [CK2], $\operatorname{PHI}(\phi)$ is obtained for symmetric jump processes of mixed types on metric measure spaces with variable scale $\phi$.

Here is the question we consider in this paper.

(Q) Suppose $(\mathcal{E}, \mathcal{F})$ and $(\widehat{\mathcal{E}}, \mathcal{F})$ are regular Dirichlet forms on $L^{2}(M ; \mu)$ of the form (1.1), whose corresponding jumping measures and processes are $J, \widehat{J}$ and $X, \widehat{X}$, respectively. Suppose further there exist constants $c_{1}, c_{2}>0$ such that $c_{1} J(A, B) \leq \widehat{J}(A, B) \leq$ $c_{2} J(A, B)$ for all $A, B \subset M$ with $A \cap B=\emptyset$. If $\operatorname{PHI}(\phi)$ holds for $X$, does $\operatorname{PHI}(\phi)$ also hold for the process $\widehat{X}$ ?

Assume the metric measure space $(M, d, \mu)$ satisfies the volume doubling and reversed volume doubling condition; see Definition 1.4 for a precise definition. In Theorem 1.20, the main result of this paper, we will not only answer the question affirmatively but also give an equivalent characterization of $\operatorname{PHI}(\phi)$ that is stable under such perturbations:

$$
\operatorname{PHI}(\phi) \Longleftrightarrow \operatorname{PI}(\phi)+\mathrm{J}_{\phi, \leq}+\mathrm{CSJ}(\phi)+\mathrm{UJS} ;
$$

see (1.22), (1.13), (1.14) and (1.21) below for related notations and definitions. Moreover, Theorem 1.20 also gives the precise relations among the parabolic Harnack inequality $\mathrm{PHI}(\phi)$, the Hölder regularity $\operatorname{PHR}(\phi)$ of caloric functions, and the elliptic Hölder regularity (EHR) of harmonic functions:

$$
\operatorname{PHI}(\phi) \Longleftrightarrow \operatorname{PHR}(\phi)+\mathrm{E}_{\phi}+\mathrm{UJS} \Longleftrightarrow \mathrm{EHR}+\mathrm{E}_{\phi}+\mathrm{UJS} ;
$$

see (1.16), (1.18) and (1.19) for definitions. As we will see from Examples 1.2 [1.3, characterization (1.3) also gives us an effective tool to establish $\operatorname{PHI}(\phi)$ for a class of symmetric jump processes.

To our knowledge, there has been no literature on the equivalence of parabolic Harnack inequalities for non-local Dirichlet forms on general metric measure spaces despite of the importance of parabolic Harnack inequalities. We note that when the underlying space is a graph satisfying the Ahlfors regular condition, some equivalence conditions for $\mathrm{PHI}(\phi)$ with $\phi(r)=r^{\alpha}$ for $\alpha \in(0,2)$ are obtained in Barlow, Bass and Kumagai [BBK2]. In some general metric measure spaces including certain fractals mentioned above, it is known that $\operatorname{PHI}(\phi)$ may hold for $\phi(r)=r^{\alpha}$ with $\alpha \geq 2$ (see, for instance, [CKW1, Section 6.1]). In this paper, we establish the stability of $\operatorname{PHI}(\phi)$ for a large class of scale functions $\phi$ including those $\phi(r)=r^{\alpha}$ with $\alpha \geq 2$. We also emphasize that our metric measure spaces are only assumed to satisfy general volume doubling and reverse volume doubling properties; see Definition 1.4 for definitions. These make the study of stability of $\mathrm{PHI}(\phi)$ extremely challenging. 
The characterization (1.3) in particular implies that $\operatorname{PHI}(\phi)$ is invariant under time change of the symmetric jump process $X$ by a positive continuous additive functional $A_{t}=\int_{0}^{t} q\left(X_{s}\right) d s$ for some measurable function $q$ that is bounded between two positive constants. This is because the time-changed process $\left(Y_{t}\right)_{t \geq 0}=\left(X_{\tau_{t}}\right)_{t \geq 0}$ is an $m$-symmetric jump process on $M$ having the same jumping kernel $J(x, y)$, where $\tau_{t}=\inf \left\{s>0: A_{s}>t\right\}$ and $m(d x):=q(x) \mu(d x)$. Clearly the right hand side of (1.3) holds for $(M, d, \mu)$ and $J$ if and only if it holds for $(M, d, m)$ and $J$.

We point out that the characterization (1.3) of $\mathrm{PHI}(\phi)$ is new even in the Euclidean space case. Suppose that $(M, d)$ is the Euclidean space $\mathbb{R}^{d}, \mu$ is a measure on $\mathbb{R}^{d}$ that is comparable to the Lebesgue measure, and $\phi(r)=\int_{\alpha_{1}}^{\alpha_{2}} r^{\beta} \nu(d \beta)$ or $\phi(r)=1 / \int_{\alpha_{1}}^{\alpha_{2}} r^{-\beta} \nu(d \beta)$, where $0<\alpha_{1}<\alpha_{2}<2$ and $\nu$ is a probability measure on $\left[\alpha_{1}, \alpha_{2}\right]$. Then, as a special case of [CKW1, Rermark 1.7], $\operatorname{CSJ}(\phi)$ is implied by $\mathrm{J}_{\phi, \leq}$. In this case, our result (1.3) says that

$$
\operatorname{PHI}(\phi) \Longleftrightarrow \operatorname{PI}(\phi)+\mathrm{J}_{\phi, \leq}+\mathrm{UJS} .
$$

In [BBK2, Theorem 1.6], (1.4) is proved for continuous time random walks on graphs that satisfy the Ahlfors $d$-regular condition with $\phi(r)=r^{\alpha}$ for $\alpha \in(0,2)$. We will illustrate the utility of (1.4) in Examples 1.2 and 1.3 below.

Parabolic Harnack inequalities are closely related to heat kernel estimates. In the recent paper [CKW1], we obtained stability of two-sided heat kernel estimates and upper bound heat kernel estimates for symmetric jump processes of mixed types on general metric measure spaces (see Section 1.2 for a brief survey of the results of [CKW1]). There are also recent work on the stability of two-sided heat kernel estimates for stable-like jumps processes with Ahlfors $d$-set condition in the framework of metric measure spaces [GHH and in the framework of infinite connected locally finite graphs [MS]. In contrast to the cases of local operators/diffusions, parabolic Harnack inequalities are no longer equivalent to (in fact weaker than) the two-sided heat kernel estimates. In fact Corollary 1.21 of this paper asserts

$$
\mathrm{HK}(\phi) \Longleftrightarrow \operatorname{PHI}(\phi)+\mathrm{J}_{\phi, \geq}
$$

see (1.13) and (1.17) for definitions. This discrepancy is caused by the heavy tail of the jumping kernel. This heavy tail phenomenon is also one of main sources of difficulties in analyzing non-local operators/jump processes.

Due to the above difficulties and differences, obtaining the stability of $\mathrm{PHI}(\phi)$ for nonlocal operators/jump processes requires new ideas. Our approach contains the following two key ingredients, and both of them are highly non-trivial:

(i) We make full use of the probabilistic properties of jump process $X$ (in particular the Lévy system of $X$ that describes how the process $X$ jumps) to connect $\operatorname{PHI}(\phi)$ with the properties of the associated heat kernel and jumping kernel. For instance, UJS yielded by a probabilistic consideration and motivated by BBK2 in a graph setting plays one of key roles for the characterization of $\operatorname{PHI}(\phi)$ in the present framework; see the main result of this paper, Theorem 1.20 .

(ii) We adopt some PDE's techniques from the recent study of fractional $p$-Laplacian operators in [CKP1] to derive some useful properties of the process $X$. We emphasis that, 
to get the stability of $\mathrm{PHI}(\phi)$ in our general framework we should use cutoff Sobolev inequalities $\operatorname{CSJ}(\phi)$ for non-local Dirichlet forms, instead of the fractional Poincaré inequalities or Sobolev inequalities in the existing literature (e.g. see [CKP1, DK, K2]), since the latter two functional inequalities require some regularity of state space and non-local operators. See the equivalence condition (7) in Theorem 1.20.

The following example, partly motivated by [BBK2] in a graph setting, illustrates the power of (1.3) characterizing $\operatorname{PHI}(\phi)$ even in the Euclidean space case. In this example, although for each fixed $x \in \mathbb{R}^{d}$, the jumping kernel $J(x, y)$ vanishes outside a double cone in $\mathbb{R}^{d}$ with apex at $x, \operatorname{PHI}(\phi)$ holds nevertheless. It also clearly indicates that only $\mathrm{PHI}(\phi)$ can not imply $\operatorname{HK}(\phi)$ without additional condition on the lower bound of the jumping kernel $J$.

Example 1.2. (PHI $(\phi)$ holds but $\operatorname{HK}(\phi)$ fails.) Let $M=\mathbb{R}^{d}, \mu$ be the Lebesgue measure on $\mathbb{R}^{d}, 0<\alpha<2$ and $\phi(r)=r^{\alpha}$. For $0<\theta<\pi / 2$ and $v \in \mathbb{R}^{d}$ with $|v|=1$, define $A=\left\{h \in \mathbb{R}^{d}:|(h /|h|, v)| \geq \cos \theta\right\}$ and

$$
J(x, y)=\mathbf{1}_{A}(x-y)|x-y|^{-d-\alpha} .
$$

We can apply (1.4) to show that $\operatorname{PHI}(\phi)$ holds. Clearly $\mathrm{J}_{\phi, \geq}$ does not hold and so $\operatorname{HK}(\phi)$ fails. In particular, caloric functions of the corresponding symmetric jump process are jointly Hölder continuous. See Section 5 for details.

When $d=2$, the above example is a special case of the following example where the direction of the cones can vary but $\operatorname{PHI}(\phi)$ still holds. Note that, the non-degenerate part of the jumping kernel in the example below can not only be of stable-like but also be of mixed stable-like type considered in [CK2].

Example 1.3. Let $M=\mathbb{R}^{2}, \mu$ be a measure on $\mathbb{R}^{2}$ that is comparable to the Lebesgue measure, and $\phi(r)=1 / \int_{\alpha_{1}}^{\alpha_{2}} r^{-\beta} \nu(d \beta)$, where $0<\alpha_{1}<\alpha_{2}<2$ and $\nu$ is a probability measure on $\left[\alpha_{1}, \alpha_{2}\right]$. Fix $\theta \in(0, \pi / 2)$ and a positive constant $c_{\theta}>0$. Let $\xi$ be an increasing function on $\mathbb{R}$ so that $\xi(0) \in[0,2 \pi), \xi\left(x+c_{\theta}\right)=\xi(x)+2 \pi$ for every $x \in \mathbb{R}$, and

$$
\xi(x+r)-\xi(x) \in\left[\sin ^{-1} r, \theta\right] \quad \text { for any } x \in \mathbb{R} \text { and } r \in\left[0, r_{\theta}\right]
$$

with some $r_{\theta} \in(0, \sin \theta)$ that is small enough. Define $v(x)=\left(\cos \left(\xi\left(x_{1}\right)\right), \sin \left(\xi\left(x_{1}\right)\right)\right)$ for $x=\left(x_{1}, x_{2}\right) \in \mathbb{R}^{2}$, and a two-sided cone $\Gamma_{\theta}(x)$ with apex angle $2 \theta$ and vertex at $x$ by

$$
\Gamma_{\theta}(x)=x+\left\{h \in \mathbb{R}^{d}:|\langle h /|h|, v(x)\rangle| \geq \cos \theta\right\} .
$$

Note that $v(x)$ is a unit vector that depends on the first coordinate $x_{1}$ of $x$ and is $c_{\theta}$-periodic in $x_{1}$.

Let $J(x, y)$ be any measurable symmetric kernel on $\mathbb{R}^{d} \times \mathbb{R}^{d}$ with the following property; there exists a constant $C \geq 1$ such that for all $x, y \in \mathbb{R}^{d}$,

$$
C^{-1} \frac{\mathbf{1}_{\Xi(x)}(y)+\mathbf{1}_{\Xi(y)}(x)}{|x-y|^{d} \phi(|x-y|)} \leq J(x, y) \leq C \frac{\mathbf{1}_{\Xi(x)}(y)+\mathbf{1}_{\Xi(y)}(x)}{|x-y|^{d} \phi(|x-y|)},
$$

where $\Xi(x):=\Gamma_{\theta}(x) \cup \overline{B(x, 1)}$. We can again apply (1.4) to show that $\operatorname{PHI}(\phi)$ holds. Consequently, bounded caloric functions for the corresponding symmetric jump process are jointly Hölder continuous. See Section 5 for details. 
In addition, we show in Example 5.2 that the trace process of Brownian motion on the Sierpinski gasket on one side of the big triangle enjoys a bounded version of $\mathrm{PHI}\left(r^{\alpha}\right)$ for some $\alpha \in(1,2)$ but $\operatorname{HK}\left(r^{\alpha}\right)$ fails on any bounded time interval $\left(0, T_{0}\right]$.

Finally, we should mention that, even though non-local operators appear naturally in the study of stochastic processes with jumps, there are huge amount of interests among analysts to study Harnack inequalities and related properties for non-local operators; see [CS, CKP1, CKP2, DK, K1, K2, Sil and the references therein. Combining probabilistic methods with analytic methods in the study of heat kernel estimates and parabolic Harnack inequalities for non-local operators proves to be quite powerful and fruitful, as is the case for this paper and for CKW1.

In the following, we give the framework of this paper in details and present the main results of this paper. We also recall some theorems from [CKW1 that will be used in this paper.

\section{$1.1 \quad$ Setting}

Let $(M, d)$ be a locally compact separable metric space, and $\mu$ a positive Radon measure on $M$ with full support. A triple $(M, d, \mu)$ is called a metric measure space, and we denote by $\langle\cdot, \cdot\rangle$ the inner product in $L^{2}(M ; \mu)$. For simplicity, we assume that $\mu(M)=\infty$ throughout the paper. (See Remark 1.22 below for further comments.) Let us emphasize that we do not assume $M$ to be connected nor $(M, d)$ to be geodesic.

Let $(\mathcal{E}, \mathcal{F})$ be a regular Dirichlet form on $L^{2}(M ; \mu)$ given in (1.1). We assume throughout this paper that, for each $x \in M$, there is a kernel $J(x, d y)$ so that

$$
J(d x, d y)=J(x, d y) \mu(d x) .
$$

In this paper, we will abuse notation and always take the quasi-continuous version for an element of $\mathcal{F}$ (note that since $(\mathcal{E}, \mathcal{F}$ ) is regular, each function in $\mathcal{F}$ admits a quasi-continuous version). Denote by $\mathcal{L}$ the (negative definite) $L^{2}$-generator of $(\mathcal{E}, \mathcal{F})$. Let $\left\{P_{t}\right\}$ be the associated semigroup on $L^{2}(M ; \mu)$. Associated with the regular Dirichlet form $(\mathcal{E}, \mathcal{F})$ on

$L^{2}(M ; \mu)$ is an $\mu$-symmetric Hunt process $X=\left\{X_{t}, t \geq 0, \mathbb{P}^{x}, x \in M \backslash \mathcal{N}\right\}$, where $\mathcal{N}$ is a properly exceptional set for $(\mathcal{E}, \mathcal{F})$ in that $\mu(\mathcal{N})=0$ and $\mathbb{P}^{x}\left(X_{t} \in \mathcal{N}\right.$ for some $\left.t>0\right)=0$ for all $x \in M \backslash \mathcal{N}$. This Hunt process is unique up to a properly exceptional set (see [FOT, Theorem 4.2.8]). A more precise version of $\left\{P_{t}\right\}$ with better regularity properties can be obtained as follows: for any bounded Borel measurable function $f$ on $M$,

$$
P_{t} f(x)=\mathbb{E}^{x} f\left(X_{t}\right), \quad x \in M_{0}:=M \backslash \mathcal{N} .
$$

The heat kernel associated with $\left\{P_{t}\right\}$ (if it exists) is a measurable function $p(t, x, y): M_{0} \times$ $M_{0} \rightarrow(0, \infty)$ for every $t>0$, such that

$$
\begin{aligned}
& \mathbb{E}^{x} f\left(X_{t}\right)=P_{t} f(x)=\int p(t, x, y) f(y) \mu(d y), \quad x \in M_{0}, f \in L^{\infty}(M ; \mu), \\
& p(t, x, y)=p(t, y, x) \quad \text { for all } t>0, x, y \in M_{0}
\end{aligned}
$$




$$
p(s+t, x, z)=\int p(s, x, y) p(t, y, z) \mu(d y) \quad \text { for all } s, t>0 \text { and } x, z \in M_{0} .
$$

We call $p(t, x, y)$ the heat kernel on $(M, d, \mu, \mathcal{E})$. Note that we can extend $p(t, x, y)$ to all $x$, $y \in M$ by setting $p(t, x, y)=0$ if $x$ or $y$ is outside $M_{0}$.

The goal of this paper is to present stable characterizations of parabolic Harnack inequality for the symmetric jump process $X$. To state our results precisely and show the relations between heat kernel estimates and parabolic Harnack inequalities, we need a number of definitions and also recall the stable characterizations of two-sided estimates and upper bound estimates for heat kernels from [CKW1.

Definition 1.4. Denote by $B(x, r)$ the ball in $(M, d)$ centered at $x$ with radius $r$, and set

$$
V(x, r)=\mu(B(x, r)) .
$$

(i) We say that $(M, d, \mu)$ satisfies the volume doubling property (VD) if there exists a constant $C_{\mu} \geq 1$ such that for all $x \in M$ and $r>0$,

$$
V(x, 2 r) \leq C_{\mu} V(x, r) .
$$

(ii) We say that $(M, d, \mu)$ satisfies the reverse volume doubling property (RVD) if there exist positive constants $d_{1}$ and $c_{\mu}$ such that for all $x \in M$ and $0<r \leq R$,

$$
\frac{V(x, R)}{V(x, r)} \geq c_{\mu}\left(\frac{R}{r}\right)^{d_{1}} \text {. }
$$

VD condition (1.7) is equivalent to the following: there exist $d_{2}, \widetilde{C}_{\mu}>0$ so that

$$
\frac{V(x, R)}{V(x, r)} \leq \widetilde{C}_{\mu}\left(\frac{R}{r}\right)^{d_{2}} \quad \text { for all } x \in M \text { and } 0<r \leq R .
$$

RVD condition (1.8) is equivalent to the existence of positive constants $l_{\mu}$ and $\widetilde{c}_{\mu}>1$ so that

$$
V\left(x, l_{\mu} r\right) \geq \widetilde{c}_{\mu} V(x, r) \quad \text { for all } x \in M \text { and } r>0 .
$$

It is known that VD implies RVD if $M$ is connected and unbounded (see, for example GH, Proposition 5.1 and Corollary 5.3]).

Let $\mathbb{R}_{+}:=[0, \infty)$ and $\phi: \mathbb{R}_{+} \rightarrow \mathbb{R}_{+}$be a strictly increasing continuous function with $\phi(0)=0, \phi(1)=1$ that satisfies the following: there exist $c_{1}, c_{2}>0$ and $\beta_{2} \geq \beta_{1}>0$ such that

$$
c_{1}\left(\frac{R}{r}\right)^{\beta_{1}} \leq \frac{\phi(R)}{\phi(r)} \leq c_{2}\left(\frac{R}{r}\right)^{\beta_{2}} \text { for all } 0<r \leq R
$$

Definition 1.5. We say $\mathrm{J}_{\phi}$ holds if for any $x, y \in M$ there exists a non-negative symmetric function $J(x, y)$ so that for $\mu \times \mu$-almost all $x, y \in M$,

$$
J(d x, d y)=J(x, y) \mu(d x) \mu(d y),
$$

and

$$
\frac{c_{1}}{V(x, d(x, y)) \phi(d(x, y))} \leq J(x, y) \leq \frac{c_{2}}{V(x, d(x, y)) \phi(d(x, y))}
$$

for some constants $c_{2} \geq c_{1}>0$. We say that $\mathrm{J}_{\phi, \leq}\left(\right.$ resp. $\left.\mathrm{J}_{\phi, \geq}\right)$ if (1.12) holds and the upper bound (resp. lower bound) in (1.13) holds. 
For the non-local Dirichlet form $(\mathcal{E}, \mathcal{F})$, we define the carré du-Champ operator $\Gamma(f, g)$ for $f, g \in \mathcal{F}$ by

$$
\Gamma(f, g)(d x)=\int_{y \in M}(f(x)-f(y))(g(x)-g(y)) J(d x, d y)
$$

\subsection{Heat kernel estimates}

The following $\operatorname{CSJ}(\phi)$ and $\operatorname{SCSJ}(\phi)$ conditions that control the energy of cutoff functions are first introduced in CKW1. See [CKW1, Remark 1.6] for background on these conditions. Recall that $\phi$ is a strictly increasing continuous function on $\mathbb{R}_{+}$satisfying $\phi(0)=0, \phi(1)=1$ and (1.11).

Definition 1.6. (i) Let $U \subset V$ be open sets in $M$ with $U \subset \bar{U} \subset V$. We say a nonnegative bounded measurable function $\varphi$ is a cutoff function for $U \subset V$, if $\varphi=1$ on $U, \varphi=0$ on $V^{c}$ and $0 \leq \varphi \leq 1$ on $M$.

(ii) We say that $\operatorname{CSJ}(\phi)$ holds if there exist constants $C_{0} \in(0,1]$ and $C_{1}, C_{2}>0$ such that for every $0<r \leq R$, almost all $x \in M$ and any $f \in \mathcal{F}$, there exists a cutoff function $\varphi \in \mathcal{F}_{b}:=\mathcal{F} \cap L^{\infty}(M, \mu)$ for $B(x, R) \subset B(x, R+r)$ so that

$$
\begin{aligned}
\int_{B\left(x, R+\left(1+C_{0}\right) r\right)} f^{2} d \Gamma(\varphi, \varphi) \leq & C_{1} \int_{U \times U^{*}}(f(x)-f(y))^{2} J(d x, d y) \\
& +\frac{C_{2}}{\phi(r)} \int_{B\left(x, R+\left(1+C_{0}\right) r\right)} f^{2} d \mu,
\end{aligned}
$$

where $U=B(x, R+r) \backslash B(x, R)$ and $U^{*}=B\left(x, R+\left(1+C_{0}\right) r\right) \backslash B\left(x, R-C_{0} r\right)$.

(iii) We say that $\operatorname{SCSJ}(\phi)$ holds if there exist constants $C_{0} \in(0,1]$ and $C_{1}, C_{2}>0$ such that for every $0<r \leq R$ and almost all $x \in M$, there exists a cutoff function $\varphi \in \mathcal{F}_{b}$ for $B(x, R) \subset B(x, R+r)$ so that (1.14) holds for any $f \in \mathcal{F}$.

Clearly $\operatorname{SCSJ}(\phi) \Longrightarrow \operatorname{CSJ}(\phi)$.

Remark 1.7. As is pointed out in [CKW1, Remark 1.7], under VD, (1.11) and $\mathrm{J}_{\phi, \leq}, \mathrm{SCSJ}(\phi)$ always holds if $\beta_{2}<2$, where $\beta_{2}$ is the exponent in (1.11). In particular, $\operatorname{SCSJ}(\phi)$ holds for $\phi(r)=r^{\alpha}$ always when $0<\alpha<2$.

We next introduce the Faber-Krahn inequality. For any open set $D \subset M, \mathcal{F}_{D}$ is defined to be the $\|\cdot\|_{\mathcal{E}_{1}}$-closure in $\mathcal{F}$ of $\mathcal{F} \cap C_{c}(D)$, where $\|\cdot\|_{\mathcal{E}_{1}}^{2}=\mathcal{E}(\cdot, \cdot)+\|\cdot\|_{2}^{2}$ Here $C_{c}(D)$ is the space of continuous functions on $M$ with compact support in $D$. Define

$$
\lambda_{1}(D)=\inf \left\{\mathcal{E}(f, f): f \in \mathcal{F}_{D} \text { with }\|f\|_{2}=1\right\},
$$

the bottom of the Dirichlet spectrum of $-\mathcal{L}$ on $D$.

Definition 1.8. $(M, d, \mu, \mathcal{E})$ satisfies the Faber-Krahn inequality $\mathrm{FK}(\phi)$, if there exist positive constants $C$ and $\nu$ such that for any ball $B(x, r)$ and any open set $D \subset B(x, r)$,

$$
\lambda_{1}(D) \geq \frac{C}{\phi(r)}(V(x, r) / \mu(D))^{\nu} .
$$


For a set $A \subset M$, define the exit time $\tau_{A}=\inf \left\{t>0: X_{t} \in A^{c}\right\}$.

Definition 1.9. We say that $\mathrm{E}_{\phi}$ holds if there is a constant $c_{1}>1$ such that for all $r>0$ and all $x \in M_{0}$,

$$
c_{1}^{-1} \phi(r) \leq \mathbb{E}^{x}\left[\tau_{B(x, r)}\right] \leq c_{1} \phi(r) .
$$

We say that $\mathrm{E}_{\phi, \leq}$ (resp. $\mathrm{E}_{\phi, \geq}$ ) holds if the upper bound (resp. lower bound) in the inequality above holds.

Definition 1.10. (i) We say that $\operatorname{HK}(\phi)$ holds if there exists a heat kernel $p(t, x, y)$ of the semigroup $\left\{P_{t}\right\}$ for $(\mathcal{E}, \mathcal{F})$, which has the following estimates for all $t>0$ and all $x, y \in M_{0}$,

$$
\begin{aligned}
c_{1}\left(\frac{1}{V\left(x, \phi^{-1}(t)\right)}\right. & \left.\wedge \frac{t}{V(x, d(x, y)) \phi(d(x, y))}\right) \\
& \leq p(t, x, y) \\
& \leq c_{2}\left(\frac{1}{V\left(x, \phi^{-1}(t)\right)} \wedge \frac{t}{V(x, d(x, y)) \phi(d(x, y))}\right)
\end{aligned}
$$

where $c_{1}, c_{2}>0$ are constants independent of $x, y \in M_{0}$ and $t>0$. Here $\phi^{-1}(t)$ is the inverse function of the strictly increasing function $t \mapsto \phi(t)$.

(ii) We say $\operatorname{UHK}(\phi)$ (resp. $\operatorname{LHK}(\phi)$ ) holds if the upper bound (resp. the lower bound) in (1.17) holds.

(iii) We say $\operatorname{UHKD}(\phi)$ holds if there is a constant $c>0$ such that

$$
p(t, x, x) \leq \frac{c}{V\left(x, \phi^{-1}(t)\right)} \quad \text { for all } t>0 \text { and } x \in M_{0} .
$$

It is pointed out in [CKW1, Remark 1.12] that

$$
\frac{1}{V\left(y, \phi^{-1}(t)\right)} \wedge \frac{t}{V(y, d(x, y)) \phi(d(x, y))} \asymp \frac{1}{V\left(x, \phi^{-1}(t)\right)} \wedge \frac{t}{V(x, d(x, y)) \phi(d(x, y))} .
$$

We may thus replace $V\left(x, \phi^{-1}(t)\right)$ and $V(x, d(x, y))$ by $V\left(y, \phi^{-1}(t)\right)$ and $V(y, d(x, y))$ in (1.17) by modifying the values of $c_{1}$ and $c_{2}$. On the other hand, it follows from CKW1, Theorem 1.13 and Lemma 5.6] that if $\operatorname{HK}(\phi)$ holds, then the heat kernel $p(t, x, y)$ is Hölder continuous on $(x, y)$ for every $t>0$, and so (1.17) holds for all $x, y \in M$.

We say $(\mathcal{E}, \mathcal{F})$ is conservative if its associated Hunt process $X$ has infinite lifetime. This is equivalent to $P_{t} 1=1$ a.e. on $M_{0}$ for every $t>0$.

The following are the main results of [CKW1, which will be used later in this paper.

Theorem 1.11. ([CKW1, Theorem 1.13]) Assume that the metric measure space $(M, d, \mu)$ satisfies VD and RVD, and $\phi$ satisfies (1.11). Then the following are equivalent:

(1) $\mathrm{HK}(\phi)$.

(2) $\mathrm{J}_{\phi}$ and $\mathrm{E}_{\phi}$.

(3) $\mathrm{J}_{\phi}$ and $\operatorname{SCSJ}(\phi)$.

(4) $\mathrm{J}_{\phi}$ and $\operatorname{CSJ}(\phi)$. 
Theorem 1.12. ([CKW1, Theorem 1.15]) Assume that the metric measure space $(M, d, \mu)$ satisfies VD and RVD, and $\phi$ satisfies (1.11). Then the following are equivalent:

(1) $\operatorname{UHK}(\phi)$ and $(\mathcal{E}, \mathcal{F})$ is conservative.

(2) $\operatorname{UHKD}(\phi), \mathrm{J}_{\phi, \leq}$ and $\mathrm{E}_{\phi}$.

(3) $\operatorname{FK}(\phi), \mathrm{J}_{\phi, \leq}$ and $\operatorname{SCSJ}(\phi)$.

(4) $\operatorname{FK}(\phi), \mathrm{J}_{\phi, \leq}$ and $\operatorname{CSJ}(\phi)$.

As a consequence of [CKW1, Proposition 3.1(ii)] (recalled in Proposition 2.4 of this paper), $\operatorname{LHK}(\phi)$ implies that $X$ has infinite lifetime. As is remarked in [CKW1], $\operatorname{UHK}(\phi)$ alone does not imply the conservativeness of the associated Dirichlet form $(\mathcal{E}, \mathcal{F})$.

\subsection{Parabolic Harnack inequalities}

We first give probabilistic definitions of harmonic and caloric functions in the general context of metric measure spaces.

Let $Z:=\left\{V_{s}, X_{s}\right\}_{s \geq 0}$ be the space-time process corresponding to $X$ where $V_{s}=V_{0}-s$. The filtration generated by $Z$ satisfying the usual conditions will be denoted by $\left\{\widetilde{\mathcal{F}}_{s} ; s \geq 0\right\}$. The law of the space-time process $s \mapsto Z_{s}$ starting from $(t, x)$ will be denoted by $\mathbb{P}^{(t, x)}$. For every open subset $D$ of $[0, \infty) \times M$, define $\tau_{D}=\inf \left\{s>0: Z_{s} \notin D\right\}$.

Recall that a set $A \subset[0, \infty) \times M$ is said to be nearly Borel measurable if for any probability measure $\mu_{0}$ on $[0, \infty) \times M$, there are Borel measurable subsets $A_{1}, A_{2}$ of $[0, \infty) \times M$ so that $A_{1} \subset A \subset A_{2}$ and that $\mathbb{P}^{\mu_{0}}\left(Z_{t} \in A_{2} \backslash A_{1}\right.$ for some $\left.t \geq 0\right)=0$. The collection of all nearly Borel measurable subsets of $[0, \infty) \times M$ forms a $\sigma$-field, which is called nearly Borel measurable $\sigma$-field.

Definition 1.13. (i) We say that a nearly Borel measurable function $u(t, x)$ on $[0, \infty) \times M$ is caloric (or space-time harmonic) on $D=(a, b) \times B\left(x_{0}, r\right)$ for the Markov process $X$ if there is a properly exceptional set $\mathcal{N}_{u}$ of the Markov process $X$ so that for every relatively compact open subset $U$ of $D, u(t, x)=\mathbb{E}^{(t, x)} u\left(Z_{\tau_{U}}\right)$ for every $(t, x) \in$ $U \cap\left([0, \infty) \times\left(M \backslash \mathcal{N}_{u}\right)\right)$.

(ii) A nearly Borel measurable function $u$ on $M$ is said to be subharmonic (resp. harmonic, superharmonic) in $D$ (with respect to the process $X$ ) if for any relatively compact subset $U \subset D, t \mapsto u\left(X_{t \wedge \tau_{U}}\right)$ is a uniformly integrable submartingale (resp. martingale, supermartingale) under $\mathbb{P}^{x}$ for q.e. $x \in U$.

Remark 1.14. Concerning the definition of the space-time process $Z:=\left\{V_{s}, X_{s}\right\}_{s>0}$, the time evolves as $V_{s}=V_{0}+s$ in [CK1, p. 37] and [CK2, p. 307], which is opposed to $V_{s}=V_{0}-s$ in the present paper (as well as in BBK2, CKK1, CKK2]). The advantage of using time backwards (i.e., $V_{s}=V_{0}-s$ for all $s>0$ ) is due to that $u(t, x)=P_{t} f(x)$ is an example of caloric function. Indeed, for every $(t, x) \in[0, \infty) \times M$ and bounded measurable function $f$, let $u(t, x)=P_{t} f(x)$. We have by the Markov property of $X$ that for any $\left(t_{0}, x_{0}\right) \in[0, \infty) \times M$ and $0<s<t$,

$$
\begin{aligned}
\left.\mathbb{E}^{\left(t_{0}, x_{0}\right)}\left(u\left(Z_{t}\right) \mid \widetilde{\mathcal{F}}_{s}\right)\right) & =\mathbb{E}^{x_{0}}\left(u\left(t_{0}-t, X_{t}\right) \mid \mathcal{F}_{s}\right)=\mathbb{E}^{X_{s}} u\left(t_{0}-t, X_{t-s}\right) \\
& =\mathbb{E}^{X_{s}} P_{t_{0}-t} f\left(X_{t-s}\right)=P_{t_{0}-s} f\left(X_{s}\right)=u\left(Z_{s}\right),
\end{aligned}
$$


which implies that $u(t, x)$ on $[0, \infty) \times M$ is caloric. Similarly, if the heat kernel $p(t, x, y)$ exists, then we can prove that $(t, x) \mapsto p\left(t, x, y_{0}\right)$ is caloric on $(0, \infty) \times M$ for any fixed $y_{0} \in M$. (Note that, in contrast with the present paper, $(t, x) \mapsto p\left(t_{0}-t, x, y_{0}\right)$ is caloric on $\left[0, t_{0}\right) \times M$ in the time forwards case, see [CK1, Lemma 4.5].) This causes the corresponding difference of the definition for the parabolic Hölder regularity (see Definition 1.15 (iii) below) between [CK1] and the present paper, but they are equivalent under a time-reversal.

Definition 1.15. (i) We say that the parabolic Harnack inequality $\mathrm{PHI}^{+}(\phi)$ holds for Markov the process $X$ if Definition 1.1 holds for some constants $C_{1}>0, C_{k}=k C_{1}$ for $k=2,3,4,0<C_{5}<1$ and $C_{6}>0$.

(ii) We say that the elliptic Harnack inequality (EHI) holds for the Markov process $X$ if there exist constants $c>0$ and $\delta \in(0,1)$ such that for every $x_{0} \in M, r>0$ and for every non-negative function $u$ on $M$ that is harmonic in $B\left(x_{0}, r\right)$,

$$
\operatorname{ess} \sup _{B\left(x_{0}, \delta r\right)} u \leq c \operatorname{ess} \inf _{B\left(x_{0}, \delta r\right)} u \text {. }
$$

(iii) We say that the parabolic Hölder regularity $\operatorname{PHR}(\phi)$ holds for the Markov process $X$ if there exist constants $c>0, \theta \in(0,1]$ and $\varepsilon \in(0,1)$ such that for every $x_{0} \in M$, $t_{0} \geq 0, r>0$ and for every bounded measurable function $u=u(t, x)$ that is caloric in $Q\left(t_{0}, x_{0}, \phi(r), r\right)$, there is a properly exceptional set $\mathcal{N}_{u} \supset \mathcal{N}$ so that

$$
|u(s, x)-u(t, y)| \leq c\left(\frac{\phi^{-1}(|s-t|)+d(x, y)}{r}\right)^{\theta} \operatorname{ess} \sup _{\left[t_{0}, t_{0}+\phi(r)\right] \times M}|u|
$$

for every $s, t \in\left(t_{0}+\phi(r)-\phi(\varepsilon r), t_{0}+\phi(r)\right)$ and $x, y \in B\left(x_{0}, \varepsilon r\right) \backslash \mathcal{N}_{u}$.

(vi) We say that the elliptic Hölder regularity (EHR) holds for the process $X$, if there exist constants $c>0, \theta \in(0,1]$ and $\varepsilon \in(0,1)$ such that for every $x_{0} \in M, r>0$ and for every bounded measurable function $u$ on $M$ that is harmonic in $B\left(x_{0}, r\right)$, there is a properly exceptional set $\mathcal{N}_{u} \supset \mathcal{N}$ so that

$$
|u(x)-u(y)| \leq c\left(\frac{d(x, y)}{r}\right)^{\theta} \operatorname{ess} \sup _{M}|u|
$$

for any $x, y \in B\left(x_{0}, \varepsilon r\right) \backslash \mathcal{N}_{u}$.

Clearly $\mathrm{PHI}^{+}(\phi) \Longrightarrow \mathrm{PHI}(\phi) \Longrightarrow \mathrm{EHI}$ and $\mathrm{PHR}(\phi) \Longrightarrow \mathrm{EHR}$.

Remark 1.16. (i) $\operatorname{PHI}(\phi)$ in Definition 1.1 is called a weak parabolic Harnack inequality in [BGK], in the sense that (1.2) holds for some $C_{1}, \cdots, C_{5}$. It is called a parabolic Harnack inequality in [BGK] if (1.2) holds for any choice of positive constants $C_{4}>$ $C_{3}>C_{2}>C_{1}>0,0<C_{5}<1$ with $C_{6}=C_{6}\left(C_{1}, \ldots, C_{5}\right)<\infty$. Since our underlying metric measure space may not be geodesic, one can not expect to deduce parabolic Harnack inequality from weak parabolic Harnack inequality. See [BGK] for related discussion on diffusions. 
(ii) We will show in Proposition 4.5 that under VD, RVD and (1.11), $\mathrm{PHI}^{+}(\phi)$ and $\mathrm{PHI}(\phi)$ are equivalent.

(iii) Clearly, $\operatorname{PHI}(\phi)$ holds if and only if the desired property holds for every bounded caloric function on cylinder $Q\left(t_{0}, x_{0}, C_{4} \phi(R), R\right)$. Same for $\mathrm{PHI}^{+}(\phi)$ and EHI.

(iv) Note that in the definition of $\operatorname{PHR}(\phi)$ (resp. EHR) if the inequality (1.18) (resp. (1.19) ) holds for some $\varepsilon \in(0,1)$, then it holds for all $\varepsilon \in(0,1)$ (with possibly different constant $c$ ). We take EHR for example. For every $x_{0} \in M$ and $r>0$, let $u$ be a bounded function on $M$ such that it is harmonic in $B\left(x_{0}, r\right)$. Then, for any $\varepsilon^{\prime} \in(0,1)$ and $x \in B\left(x_{0}, \varepsilon^{\prime} r\right) \backslash \mathcal{N}_{u}, u$ is harmonic on $B\left(x,\left(1-\varepsilon^{\prime}\right) r\right)$. Applying (1.19) for $u$ on $\left.B\left(x,\left(1-\varepsilon^{\prime}\right) r\right)\right)$, we find that for any $y \in B\left(x_{0}, \varepsilon^{\prime} r\right) \backslash \mathcal{N}_{u}$ with $d(x, y) \leq\left(1-\varepsilon^{\prime}\right) \varepsilon r$,

$$
|u(x)-u(y)| \leq c\left(\frac{d(x, y)}{r}\right)^{\theta} \operatorname{ess} \sup _{z \in M}|u(z)| .
$$

This implies that for any $x, y \in B\left(x_{0}, \varepsilon^{\prime} r\right) \backslash \mathcal{N}_{u}$, (1.19) holds with $c^{\prime}=c \vee \frac{2}{\left[\left(1-\varepsilon^{\prime}\right) \varepsilon\right]^{\theta}}$.

Below we discuss stability of parabolic Harnack inequalities. This requires further definitions.

Definition 1.17. We say that a near diagonal lower bounded estimate for Dirichlet heat kernel $\operatorname{NDL}(\phi)$ holds, i.e. there exist $\varepsilon \in(0,1)$ and $c_{1}>0$ such that for any $x_{0} \in M, r>0$, $0<t \leq \phi(\varepsilon r)$ and $B=B\left(x_{0}, r\right)$,

$$
p^{B}(t, x, y) \geq \frac{c_{1}}{V\left(x_{0}, \phi^{-1}(t)\right)}, \quad x, y \in B\left(x_{0}, \varepsilon \phi^{-1}(t)\right) \cap M_{0} .
$$

Under VD, we may replace $V\left(x_{0}, \phi^{-1}(t)\right)$ in the definition by either $V\left(x, \phi^{-1}(t)\right)$ or $V\left(y, \phi^{-1}(t)\right)$. Under (1.11), we also may replace $\phi(\varepsilon r)$ and $\varepsilon \phi^{-1}(t)$ in the definition above by $\varepsilon \phi(r)$ and $\phi^{-1}(\varepsilon t)$, respectively.

The following inequality was introduced in [BBK2] in the setting of graphs. See [CKK1] for the general setting of metric measure spaces.

Definition 1.18. We say that UJS holds if there is a symmetric function $J(x, y)$ so that $J(x, d y)=J(x, y) \mu(d y)$, and there is a constant $c>0$ such that for $\mu$-a.e. $x, y \in M$ with $x \neq y$,

$$
J(x, y) \leq \frac{c}{V(x, r)} \int_{B(x, r)} J(z, y) \mu(d z) \quad \text { for every } 0<r \leq d(x, y) / 2 .
$$

Note that UJS is implied by the following pointwise comparability condition of the jump kernel $J(x, y)$ : there is a constant $c>0$ such that $J(x, y) \leq c J(z, y)$ for $\mu$-a.e. $x, y, z \in M$ with $x \neq y$ and $0<d(x, z) \leq d(x, y) / 2$. Some sufficient conditions for UJS can be found in CKK2, Lemma 2.1 and Example 2.2]. 
Definition 1.19. We say that the (weak) Poincaré inequality $\mathrm{PI}(\phi)$ holds if there exist constants $C>0$ and $\kappa \geq 1$ such that for any ball $B_{r}=B(x, r)$ with $x \in M$ and for any $f \in \mathcal{F}_{b}$,

$$
\int_{B_{r}}\left(f-\bar{f}_{B_{r}}\right)^{2} d \mu \leq C \phi(r) \int_{B_{\kappa r} \times B_{\kappa r}}(f(y)-f(x))^{2} J(d x, d y),
$$

where $\bar{f}_{B_{r}}=\frac{1}{\mu\left(B_{r}\right)} \int_{B_{r}} f d \mu$ is the average value of $f$ on $B_{r}$.

If the integral on the right hand side of $(1.22)$ is over $B_{r} \times B_{r}$ (i.e. $\kappa=1$ ), then it is called strong Poincaré inequality. If the metric is geodesic, it is known that (weak) Poincaré inequality implies strong Poincaré inequality (see for instance [Sa2, Section 5.3]), but in general they are not the same. In this paper, we only use weak Poincaré inequality. Note also that the left hand side of (1.22) is equal to $\inf _{a \in \mathbb{R}} \int_{B_{r}}(f-a)^{2} d \mu$.

The following is the main result of this paper.

Theorem 1.20. Suppose that the metric measure space $(M, d, \mu)$ satisfies VD and RVD, and $\phi$ satisfies (1.11). Then the following are equivalent:

(1) $\operatorname{PHI}(\phi)$.

(2) $\mathrm{PHI}^{+}(\phi)$.

(3) $\operatorname{UHK}(\phi), \operatorname{NDL}(\phi)$ and UJS.

(4) $\operatorname{NDL}(\phi)$ and UJS.

(5) $\operatorname{PHR}(\phi), \mathrm{E}_{\phi}$ and UJS.

(6) $\mathrm{EHR}, \mathrm{E}_{\phi}$ and UJS.

(7) $\operatorname{PI}(\phi), \mathrm{J}_{\phi, \leq}, \operatorname{CSJ}(\phi)$ and UJS.

We note that any of the conditions above implies the conservativeness of the process $\left\{X_{t}\right\}$; see Proposition 2.4 and [CKW1, Lemma 4.22], Proposition 3.2 and Proposition 4.10,

As a corollary of Theorem 1.11 and Theorem 1.20 (noting that $J_{\phi}$ implies UJS), we have the following.

Corollary 1.21. Suppose that the metric measure space $(M, d, \mu)$ satisfies VD and RVD, and $\phi$ satisfies (1.11). Then

$$
\mathrm{HK}(\phi) \Longleftrightarrow \operatorname{PHI}(\phi)+\mathrm{J}_{\phi, \geq} .
$$

Remark 1.22. In this paper, the metric measure space $(M, d, \mu)$ is assumed to be unbounded. This condition can be relaxed. In fact, if all the corresponding conditions on $(M, d, \mu)$ are imposed only for a finite range of radius (that is, assumed to hold for all $r \in(0, \bar{R})$ for some $\bar{R} \in(0, \operatorname{diam} M])$, then with a minor adjustment of the proofs, all the results of this paper continue to hold but with a localized version, for instance, with the statement of $\operatorname{PHI}(\phi)$ changed to hold for all $r \in(0, \bar{R})$, and those of $\operatorname{UHK}(\phi)$ and $\operatorname{HK}(\phi)$ changed to hold for $t \in(0, \phi(\bar{R}))$ and all $x, y \in M$. In particular, all results of this paper hold on bounded metric measure spaces with the aforementioned modification. We plan to spell out the details in a future publication. We note that for the heat kernel estimates for stable-like with Ahlfors $d$-set condition, $\mathrm{GHH}$ ] considers both bounded and unbounded cases. 


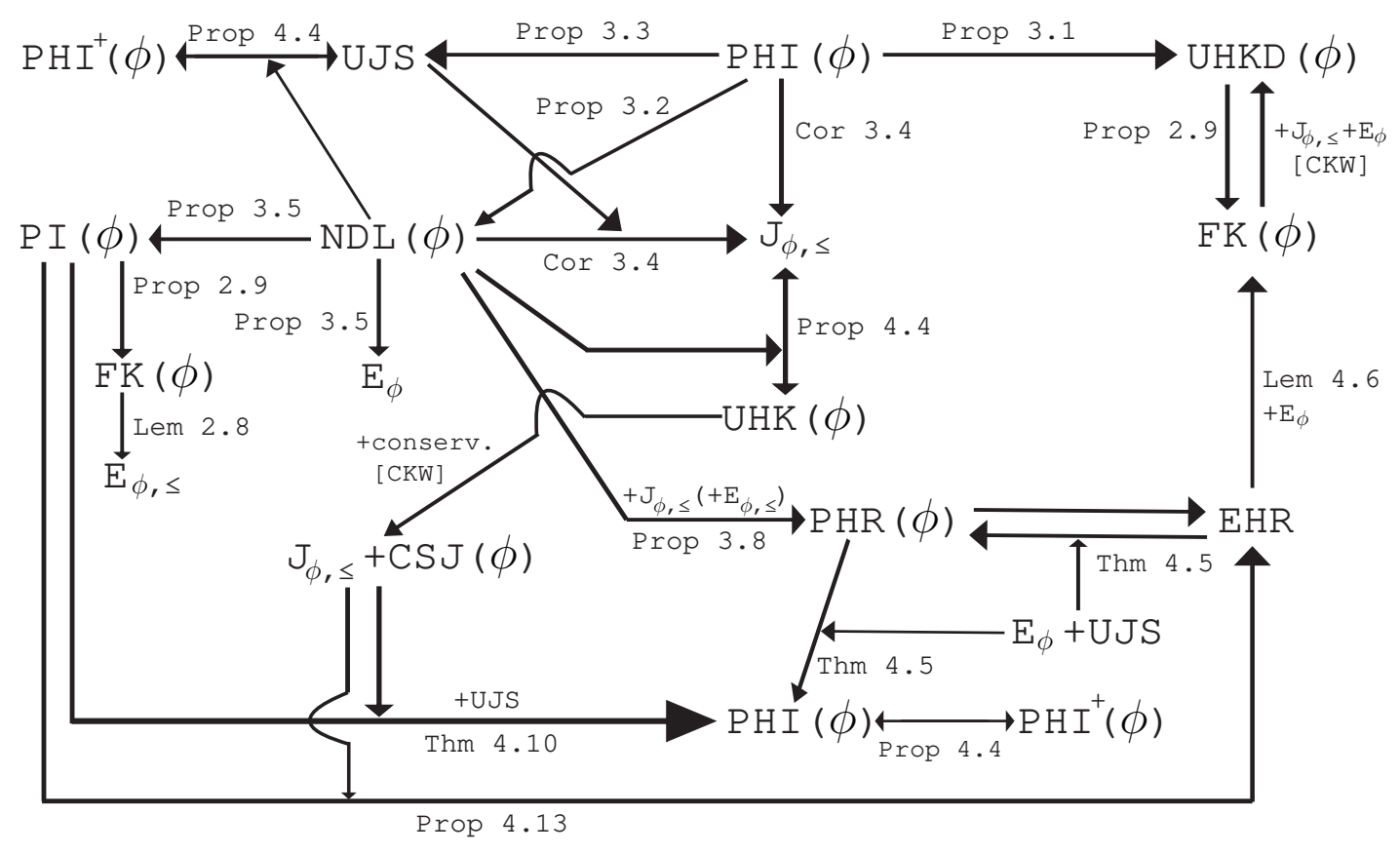

Figure 1: diagram

In this paper, we concentrate on the stability of parabolic Harnack inequalities. Stability of elliptic Harnack inequalities and the connection to the Hölder regularity of harmonic functions for symmetric non-local Dirichlet forms are studied in a separate paper [CKW3.

In addition to the papers mentioned above, for other related work on Harnack inequalities and Hölder regularities for harmonic functions of non-local operators, we mention [BL1, ChZ, LS, Kom, MK, SU, SV] and the references therein. We emphasize this is only a partial list of the vast literature on the subject.

The rest of the paper is organized as follows. The proof of Theorem 1.20 is given in Section 4. In Section 2, we present some preliminary results. Various consequences of parabolic Harnack inequalities are given in Section 3. The proof of $(1) \Longleftrightarrow(2) \Longleftrightarrow(3) \Longleftrightarrow(4)$ is given in Subsection 4.1, the proof of $(1) \Longleftrightarrow(5) \Longleftrightarrow(6)$ is given in Subsection 4.2, while $(1) \Longleftrightarrow(7)$ is shown in Subsection 4.3. Figure1 1llustrates implications of various conditions and flow of our proofs.

Throughout this paper, we will use $c$, with or without subscripts, to denote strictly positive finite constants whose values are insignificant and may change from line to line. For functions $f$ and $g$ defined on a set $D$, we write $f \asymp g$ if there exists a constant $c \geq 1$ such that $c^{-1} f(x) \leq g(x) \leq c f(x)$ for all $x \in D$. For $p \in[1, \infty]$, we will use $\|f\|_{p}$ to denote the $L^{p}$-norm in $L^{p}(M ; \mu)$. For any $D \subset M$, denote by $C(D)$ (resp. $\left.C_{c}(D)\right)$ the set of continuous functions (resp. continuous functions with compact support) on $D$. In this paper, we omit some of the proofs that are similar to those in literature. 


\section{Preliminaries}

In this section we present some preliminary results that will be used in the sequel.

We first recall the analytic characterization of harmonic and subharmonic functions. Let $D$ be an open subset of $M$. Recall that a function $f$ is said to be locally in $\mathcal{F}_{D}$, denoted as $f \in \mathcal{F}_{D}^{\text {loc }}$, if for every relatively compact subset $U$ of $D$, there is a function $g \in \mathcal{F}_{D}$ such that $f=g m$-a.e. on $U$. The following is established in $[\mathrm{C}$.

Lemma 2.1. ([C, Lemma 2.6]) Let $D$ be an open subset of $M$. Suppose $u$ is a function in $\mathcal{F}_{D}^{\text {loc }}$ that is locally bounded on $D$ and satisfies that

$$
\int_{U \times V^{c}}|u(y)| J(d x, d y)<\infty
$$

for any relatively compact open sets $U$ and $V$ of $M$ with $\bar{U} \subset V \subset \bar{V} \subset D$. Then for every $v \in C_{c}(D) \cap \mathcal{F}$, the expression

$$
\int(u(x)-u(y))(v(x)-v(y)) J(d x, d y)
$$

is well defined and finite; it will still be denoted as $\mathcal{E}(u, v)$.

As noted in [C, $(2.3)]$, since $(\mathcal{E}, \mathcal{F})$ is a regular Dirichlet form on $L^{2}(M ; \mu)$, for any relatively compact open sets $U$ and $V$ with $\bar{U} \subset V$, there is a function $\psi \in \mathcal{F} \cap C_{c}(M)$ such that $\psi=1$ on $U$ and $\psi=0$ on $V^{c}$. Consequently,

$$
\int_{U \times V^{c}} J(d x, d y)=\int_{U \times V^{c}}(\psi(x)-\psi(y))^{2} J(d x, d y) \leq \mathcal{E}(\psi, \psi)<\infty,
$$

so each bounded function $u$ satisfies (2.1).

We say that a nearly Borel measurable function $u$ on $M$ is $\mathcal{E}$-subharmonic (resp. $\mathcal{E}$ harmonic, $\mathcal{E}$-superharmonic) in $D$ if $u \in \mathcal{F}_{D}^{\text {loc }}$ that is locally bounded on $D$, satisfies (2.1) for any relatively compact open sets $U$ and $V$ of $M$ with $\bar{U} \subset V \subset \bar{V} \subset D$, and that

$$
\mathcal{E}(u, \varphi) \leq 0 \quad(\text { resp. }=0, \geq 0) \quad \text { for any } 0 \leq \varphi \in \mathcal{F} \cap C_{c}(D)
$$

The following is established in [C, Theorem 2.11 and Lemma 2.3] first for harmonic functions, and then extended in [ChK, Theorem 2.9] to subharmonic functions.

Theorem 2.2. Let $D$ be an open subset of $M$, and $u$ be a bounded function. Then $u$ is $\mathcal{E}$-harmonic (resp. $\mathcal{E}$-subharmonic) in $D$ if and only if $u$ is harmonic (resp. subharmonic) in $D$.

We next recall four results from [CKW1. Lemma 2.3 is essentially given in CK2, Lemma 2.1].

Lemma 2.3. ([CKW1, Lemma 2.1]) Assume that VD, (1.11) and $\mathrm{J}_{\phi, \leq}$ hold. Then there exists a constant $c_{1}>0$ such that

$$
\int_{B(x, r)^{c}} J(x, y) \mu(d y) \leq \frac{c_{1}}{\phi(r)} \quad \text { for every } x \in M \text { and } r>0 .
$$


Proposition 2.4. ([CKW1, Proposition 3.1(ii)]) Suppose that VD holds. Then either LHK $(\phi)$ or $\operatorname{NDL}(\phi)$ implies $\zeta=\infty$ a.s., where $\zeta$ denotes the lifetime of the process $X$.

For a Borel measurable function $u$ on $M$, following [CKP1], we define its nonlocal tail Tail $\left(u ; x_{0}, r\right)$ in the ball $B\left(x_{0}, r\right)$ by

$$
\text { Tail }\left(u ; x_{0}, r\right):=\phi(r) \int_{B\left(x_{0}, r\right)^{c}} \frac{|u(z)|}{V\left(x_{0}, d\left(x_{0}, z\right)\right) \phi\left(d\left(x_{0}, z\right)\right)} \mu(d z) .
$$

In the following, for any $x \in M$ and $r>0$, set $B_{r}(x)=B(x, r)$.

Lemma 2.5. ([CKW1, Lemma 4.8]) Suppose VD, (1.11), $\mathrm{FK}(\phi), \operatorname{CSJ}(\phi)$ and $\mathrm{J}_{\phi, \leq}$ hold. Let $x_{0} \in M, R, r_{1}, r_{2}>0$ with $r_{1} \in[R / 2, R]$ and $r_{1}+r_{2} \leq R$, and $u$ be an $\mathcal{E}$-subharmonic function in $B_{R}\left(x_{0}\right)$. For $\theta>0$, set $v:=(u-\theta)_{+}$. We have

$$
\begin{aligned}
\int_{B_{r_{1}}\left(x_{0}\right)} v^{2} d \mu \leq & \frac{c_{1}}{\theta^{2 \nu} V\left(x_{0}, R\right)^{\nu}}\left(\int_{B_{r_{1}+r_{2}}\left(x_{0}\right)} u^{2} d \mu\right)^{1+\nu} \\
& \times\left(1+\frac{r_{1}}{r_{2}}\right)^{\beta_{2}}\left[1+\left(1+\frac{r_{1}}{r_{2}}\right)^{d_{2}+\beta_{2}-\beta_{1}} \frac{\text { Tail }\left(u ; x_{0}, R / 2\right)}{\theta}\right],
\end{aligned}
$$

where $\nu$ is the constant in $\mathrm{FK}(\phi), d_{2}$ is the constant in (1.9), $\beta_{1}, \beta_{2}$ are the constants in (1.11), and $c_{1}$ is a constant independent of $\theta, x_{0}, R, r_{1}$ and $r_{2}$.

Proposition 2.6. ([CKW1, Proposition 4.10]) ( $L^{2}$-mean value inequality) Assume VD, (1.11), $\operatorname{FK}(\phi), \operatorname{CSJ}(\phi)$ and $\mathrm{J}_{\phi, \leq}$ hold. For any $x_{0} \in M$ and $r>0$, let $u$ be a bounded $\mathcal{E}$-subharmonic in $B_{r}\left(x_{0}\right)$. Then there is a constant $C_{0}>0$ independent of $x_{0}$ and $r$ so that

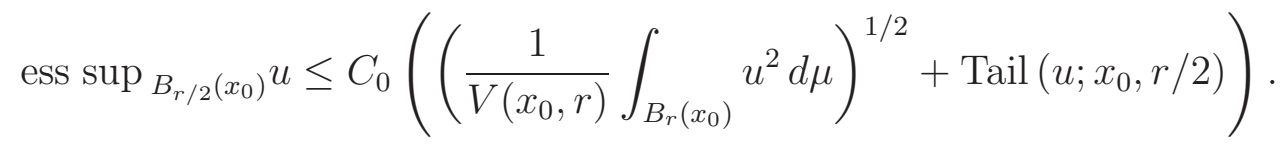

The following three results are proved in [CKW1.

Proposition 2.7. ([CKW1, Proposition 4.14]) Assume VD, (1.11), FK $(\phi), \mathrm{J}_{\phi, \leq}$ and $\mathrm{CSJ}(\phi)$ hold. Then, $\mathrm{E}_{\phi}$ holds.

Lemma 2.8. ([CKW1, Lemma 4.15]) Assume that VD, (1.11) and FK( $\phi)$ hold. Then, $\mathrm{E}_{\phi, \leq}$ holds.

Proposition 2.9. ([CKW1, Proposition 7.6]) Assume that VD, RVD and (1.11) are satisfied. Then either $\operatorname{PI}(\phi)$ or $\operatorname{UHKD}(\phi)$ implies $\mathrm{FK}(\phi)$.

We also record the following elementary iteration lemma, see, e.g., [G, Lemma 7.1] or [CKW1, Lemma 4.9].

Lemma 2.10. Let $\beta>0$ and let $\left\{A_{j}\right\}$ be a sequence of real positive numbers such that $A_{j+1} \leq c_{0} b^{j} A_{j}^{1+\beta}$ for every $j \geq 0$ with $c_{0}>0$ and $b>1$. If $A_{0} \leq c_{0}^{-1 / \beta} b^{-1 / \beta^{2}}$, then we have $A_{j} \leq b^{-j / \beta} A_{0}$ for $j \geq 1$, which in particular yields $\lim _{j \rightarrow \infty} A_{j}=0$. 
The following formula, often called the Lévy system formula, will be used many times in this paper. See, for example [CK2, Appendix A] for a proof.

Lemma 2.11. Let $f$ be a non-negative measurable function on $\mathbb{R}_{+} \times M \times M$ that vanishes along the diagonal. Then for every $t \geq 0, x \in M_{0}$ and stopping time $T$ (with respect to the filtration of $\left.\left\{X_{t}\right\}\right)$,

$$
\mathbb{E}^{x}\left[\sum_{s \leq T} f\left(s, X_{s-}, X_{s}\right)\right]=\mathbb{E}^{x}\left[\int_{0}^{T} \int_{M} f\left(s, X_{s}, y\right) J\left(X_{s}, d y\right) d s\right] .
$$

\section{Consequences of Harnack inequalities}

\subsection{Consequences of $\operatorname{PHI}(\phi)$}

In this subsection (together with some of the results from next subsection), we prove that $\operatorname{PHI}(\phi)$ implies $\operatorname{UHK}(\phi), \operatorname{NDL}(\phi)$ and UJS. Without further mention, throughout the proof we will assume that $\mu$ and $\phi$ satisfy VD and (1.11), respectively. Noting that $V(y, r)>0$ for every $y \in M$ and $r>0$ (since $\mu$ has full support), we have from (1.9) that for all $x, y \in M$ and $0<r \leq R$,

$$
\frac{V(x, R)}{V(y, r)} \leq \frac{V(y, d(x, y)+R)}{V(y, r)} \leq \widetilde{C}_{\mu}\left(\frac{d(x, y)+R}{r}\right)^{d_{2}}
$$

Proposition 3.1. Under VD and (1.11), $\operatorname{PHI}(\phi)$ implies $\operatorname{UHKD}(\phi)$.

Proof. Let $C_{i}(i=1, \ldots, 6)$ be the constants taken from the definition of $\mathrm{PHI}(\phi)$. For any $x_{0} \in M, r>0, t=C_{4} \phi(r)$ and any $0 \leq f \in L^{2}(M ; \mu) \cap L^{1}(M ; \mu)$, applying $\mathrm{PHI}(\phi)$ to the caloric function $v(s, x):=P_{s} f(x)$ in $Q\left(0, x_{0}, t, r\right)$, we have for $x, y \in B\left(x_{0}, C_{5} r\right) \backslash \mathcal{N}_{v}$,

$$
P_{\left(C_{1}+C_{2}\right) \phi(r) / 2} f(x) \leq C_{6} P_{\left(C_{3}+C_{4}\right) \phi(r) / 2} f(y),
$$

where $\mathcal{N}_{v}$ is the properly exceptional set associated with $v$. Then,

$$
V\left(x_{0}, C_{5} r\right) P_{\left(C_{1}+C_{2}\right) \phi(r) / 2} f(x) \leq C_{6} \int_{B\left(x_{0}, C_{5} r\right)} P_{\left(C_{3}+C_{4}\right) \phi(r) / 2} f(y) \mu(d y) \leq C_{6} \int f(y) \mu(d y) .
$$

Therefore, there is a constant $c_{1}>0$ such that for almost all $x \in M$ and $t>0$,

$$
P_{t} f(x) \leq \frac{c_{1}}{V\left(x, \phi^{-1}(t)\right)}\|f\|_{1},
$$

where we have used VD and (1.11) in the inequality above. In particular, the semigroup $\left\{P_{t}\right\}$ is locally ultracontractive. According to [CKW1, Proposition 7.7] (see also [BBCK, Theorem 3.1] and [GT, Theorem 2.12]), there exists a properly exceptional set $\mathcal{N} \subset M$ such that, the semigroup $\left\{P_{t}\right\}$ possesses the heat kernel $p(t, x, y)$ with domain $(0, \infty) \times(M \backslash \mathcal{N}) \times(M \backslash \mathcal{N})$.

By (3.2) again, for almost all $x, y \in M$,

$$
p(t, x, y) \leq \frac{c_{1}}{V\left(x, \phi^{-1}(t)\right)}
$$


In the following, for any $x \in M$ and $t>0$, define

$$
\varphi(x, t)=\inf _{0<r \leq \phi^{-1}(t)} \frac{1}{\mu(B(x, r))} \int_{B(x, r)} \frac{1}{V\left(z, \phi^{-1}(t)\right)} \mu(d z) .
$$

On the one hand, by (3.1) from VD, there is a constant $c_{2}>1$ such that for all $x \in M$ and $t>0$,

$$
\frac{1}{c_{2} V\left(x, \phi^{-1}(t)\right)} \leq \varphi(x, t) \leq \frac{c_{2}}{V\left(x, \phi^{-1}(t)\right)} .
$$

On the other hand, for any $t>0, x \mapsto \varphi(x, t)$ is an upper semi-continuous function on $M$. Indeed, for any $x \in M$,

$$
\begin{aligned}
\limsup _{y \rightarrow x} \varphi(y, t) & =\lim _{s \rightarrow 0} \sup _{0<d(y, x) \leq s} \inf _{0<r \leq \phi^{-1}(t)} \frac{1}{\mu(B(y, r))} \int_{B(y, r)} \frac{1}{V\left(z, \phi^{-1}(t)\right)} \mu(d z) \\
& \leq \inf _{0<r \leq \phi^{-1}(t)} \lim _{s \rightarrow 0} \sup _{0<d(y, x) \leq s} \frac{1}{\mu(B(y, r))} \int_{B(y, r)} \frac{1}{V\left(z, \phi^{-1}(t)\right)} \mu(d z) \\
& =\inf _{0<r \leq \phi^{-1}(t)} \frac{1}{\mu(B(x, r))} \int_{B(x, r)} \frac{1}{V\left(z, \phi^{-1}(t)\right)} \mu(d z) \\
& =\varphi(x, t) .
\end{aligned}
$$

Combining all the conclusions above with [CKW1, Proposition 7.7] again, we have

$$
p(t, x, y) \leq \frac{c_{3}}{V\left(x, \phi^{-1}(t)\right)} \quad \text { for all }(x, y) \in(M \backslash \mathcal{N}) \times(M \backslash \mathcal{N})
$$

This proves $\operatorname{UHKD}(\phi)$.

A key consequence of $\operatorname{PHI}(\phi)$ is a near-diagonal lower bound estimate for $p^{D}(t, x, y)$. For the cases of diffusions, similar fact was proved in [BGK, Section 4.3.4], but there is a gap in the middle of Page 1129. (Indeed, the proof uses $B\left(x_{0}, R+\rho\right)=\cup_{x \in B\left(x_{0}, R\right)} B(x, \rho)$, which is not true in general unless the metric is geodesic.) Our proof below fixes the issue (see step (ii) in the proof) and proves $\operatorname{NDL}(\phi)$ in the framework of general metric spaces.

Proposition 3.2. Assume VD, (1.11) and $\mathrm{PHI}(\phi)$ hold. Then $\mathrm{NDL}(\phi)$ holds. Consequently, $X=\left\{X_{t}\right\}$ is conservative.

Proof. Note that by VD and Proposition 2.4. NDL $(\phi)$ implies the conservativeness of the process $X$. We only need to verify that $\operatorname{NDL}(\phi)$ holds. Below we will prove NDL $(\phi)$ with $\phi(\varepsilon r)$ and $\varepsilon \phi^{-1}(t)$ replaced by $\varepsilon \phi(r)$ and $\phi^{-1}(\varepsilon t)$ in the definition.

(i) For any open ball $B:=B\left(x_{0}, r\right)$ with $x_{0} \in M_{0}$ and $r>0$, it follows from (3.2) and VD that for any $t>0$

$$
\left\|P_{t}^{B} f\right\|_{\infty} \leq \frac{c_{1}}{V\left(x_{0}, \phi^{-1}(t)\right)}\|f\|_{1}
$$

Then, by $\mathrm{BBCK}$, Theorem 3.1], the Dirichlet semigroup $\left\{P_{t}^{B}\right\}$ has the heat kernel $p^{B}(t, x, y)$ defined on $(0, \infty) \times\left(B \backslash \mathcal{N}_{1}\right) \times\left(B \backslash \mathcal{N}_{1}\right)$ such that

$$
p^{B}(t, x, y) \leq \frac{c_{1}}{V\left(x_{0}, \phi^{-1}(t)\right)}, \quad x, y \in B \backslash \mathcal{N}_{1},
$$


where $\mathcal{N}_{1} \subset B$ is a properly exceptional set of the killing process $\left\{X_{t}^{B}\right\}$ such that $\mathcal{N}_{1} \supset \mathcal{N} \cap B$; moreover, there is an $\mathcal{E}^{B}$-nest $\left\{F_{k}\right\}$ consisting of an increasing sequence of compact sets of $B$ so that $\mathcal{N}_{1}=B \backslash \cup_{k=1}^{\infty} F_{k}$ and that for every $t>0, y \in B \backslash \mathcal{N}$ and $k \geq 1, x \mapsto p^{B}(t, x, y)$ is continuous on each $F_{k}$ (i.e. for every $t>0$ and $y \in B \backslash \mathcal{N}_{1}$, the function $x \mapsto p^{B}(t, x, y)$ is quasi-continuous on $B)$.

(ii) Choose an $\widehat{x}_{0} \in B\left(x_{0}, C_{5} r\right) \backslash \mathcal{N}_{1}$, where $C_{5} \in(0,1)$ is the constant in $\operatorname{PHI}(\phi)$. Define

$$
\widehat{B}=\left\{y \in B \backslash \mathcal{N}_{1}: p^{B}\left(t, \widehat{x}_{0}, y\right)>0 \text { for some } t>0\right\} .
$$

We will show that for every $x, y \in \widehat{B}$, there is some $t>0$ so that $p^{B}(t, x, y)>0$, and that

$$
p^{B}(t, x, y)=0 \quad \text { on }(0, \infty) \times \widehat{B} \times\left(B \backslash\left(\widehat{B} \cup \mathcal{N}_{1}\right)\right) .
$$

To prove these, first noting that since $\mathbb{P}^{x}\left(\lim _{t \downarrow 0} X_{t}^{B}=X_{0}^{B}=x\right)=1$ implies $\mathbb{P}^{x}\left(\tau_{B}>0\right)=1$, we must have $p^{B}\left(t, \widehat{x}_{0}, \widehat{x}_{0}\right)=\int_{B} p^{B}\left(t / 2, \widehat{x}_{0}, y\right)^{2} \mu(d y)>0$ for some $t>0$. Thus $\widehat{x}_{0} \in \widehat{B}$. By $\operatorname{PHI}(\phi)$ applied to the caloric function $(s, y) \mapsto p^{B}\left(s, y, \widehat{x}_{0}\right)=p^{B}\left(s, \widehat{x}_{0}, y\right)$, we see that if $x \in \widehat{B}$, then there are constants $r_{x}>0$ and $s_{x}>0$ so that

$$
p^{B}\left(s, \widehat{x}_{0}, z\right)>0 \quad \text { for every } z \in B\left(x, r_{x}\right) \backslash \mathcal{N}_{1} \text { and } s \geq s_{x} .
$$

Hence, there is an open subset $U$ of $B$ containing $\widehat{x}_{0}$ so that $\widehat{B}=U \backslash \mathcal{N}_{1}$. Similarly, for every $x, y \in \widehat{B}$, by $\operatorname{PHI}(\phi)$, there are constants $r_{0}>0$ and $s_{0}>0$ so that

$$
p^{B}(s, x, z)>0 \quad \text { and } \quad p^{B}(s, y, z)>0 \quad \text { for every } z \in B\left(\widehat{x}_{0}, r_{0}\right) \backslash \mathcal{N}_{1} \text { and } s \geq s_{0} .
$$

In particular, it follows that for every $s, t \geq s_{0}$,

$$
p^{B}(t+s, x, y) \geq \int_{B\left(\widehat{x}_{0}, r_{0}\right)} p^{B}(s, x, z) p^{B}(t, z, y) \mu(d z)>0 .
$$

For $x \in \widehat{B}$, define

$$
\widehat{B}_{x}=\left\{y \in B \backslash \mathcal{N}_{1}: p^{B}(t, x, y)>0 \text { for some } t>0\right\} .
$$

Then $\widehat{B} \subset \widehat{B}_{x}$. We claim $\widehat{B}=\widehat{B}_{x}$. Were $\widehat{B} \varsubsetneqq \widehat{B}_{x}$, take $y \in \widehat{B}_{x} \backslash \widehat{B}$. By PHI $(\phi)$ applied to the caloric function $(s, z) \mapsto p^{B}(s, z, y)=p^{B}(s, y, z)$, there are constants $r_{x}>0$ and $s_{x}>0$ so that $p^{B}(s, y, z)>0$ for every $z \in B\left(x, r_{x}\right) \backslash \mathcal{N}_{1}$ and $s \geq s_{x}$, and (3.4) holds. Hence, for every $t, s \geq s_{x}$, we have

$$
p^{B}\left(t+s, \widehat{x}_{0}, y\right) \geq \int_{B\left(x, r_{x}\right)} p^{B}\left(t, \widehat{x}_{0}, z\right) p^{B}(s, z, y) \mu(d z)>0,
$$

which implies that $y \in \widehat{B}$. This contradiction shows that $\widehat{B}_{x}=\widehat{B}$ for every $x \in \widehat{B}$. We have thus established that for every $x, y \in \widehat{B}$, there is some $t>0$ so that $p^{B}(t, x, y)>0$, and that (3.3) holds. Consequently, for every $t>0$ and $x, y \in \widehat{B}=U \backslash \mathcal{N}_{1}$,

$$
p^{U}(t, x, y)=p^{B}(t, x, y)-\mathbb{E}_{x}\left[p^{B}\left(t-\tau_{U}, X_{\tau_{U}}^{B}, y\right) ; t<\tau_{U}\right]=p^{B}(t, x, y)
$$


Observe that by the symmetry of $p^{B}(t, x, y)$, (3.3) implies that

$$
\int_{B \backslash U} P_{t}^{B} \mathbf{1}_{U}(x) \mu(d x)=\int_{U \times(B \backslash U)} p^{B}(t, x, y) \mu(d x) \mu(d y)=0 ;
$$

in other words, for every $t>0$,

$$
P_{t}^{B} \mathbf{1}_{U}=0 \quad \mu \text {-a.e. on } B \backslash U \text {. }
$$

Let $\lambda_{0}>0$ be the bottom of the generator $\mathcal{L}^{U}$ associated with $\left\{P_{t}^{U}\right\}$ and $\psi \geq 0$ the corresponding eigenfunction with $\|\psi\|_{L^{2}(U ; \mu)}=1$. Note that $\psi=0$ on $B \backslash U$. In view of (3.6) and (3.7), we have for every $t>0$ and $x \in B \backslash \mathcal{N}_{1}$,

$$
P_{t}^{B} \psi(x)=P_{t}^{U} \psi(x)=e^{-\lambda_{0} t} \psi(x)
$$

Since

$$
e^{-\lambda_{0} t}\|\psi\|_{L^{\infty}(B ; \mu)}=\left\|P_{t}^{B} \psi\right\|_{L^{\infty}(B ; \mu)} \leq \mu(B)\|\psi\|_{L^{\infty}(B ; \mu)} \sup _{x, y \in B \backslash \mathcal{N}_{1}} p^{B}(t, x, y),
$$

we have

$$
\sup _{x, y \in B \backslash \mathcal{N}_{1}} p^{B}(t, x, y) \geq \frac{1}{\mu(B)} e^{-\lambda_{0} t} .
$$

We claim that $\psi>0$ on $\widehat{B}$. Noticing that

$$
v(t, x):=P_{t}^{B} \psi(x)=e^{-\lambda_{0} t} \psi(x)
$$

is a caloric function on $(0, \infty) \times B$ and $\psi>0$ has unit $L^{2}(B ; \mu)$-norm, by $\operatorname{PHI}(\phi)$, there are some $y_{0} \in \widehat{B}$ and $r_{0}>0$ so that $B\left(y_{0}, r_{0}\right) \backslash \mathcal{N}_{1} \subset \widehat{B}$, and $\psi>0$ on $B\left(y_{0}, r_{0}\right)$. On the other hand, for every $x \in \widehat{B}$, by (3.5) (and so $p^{B}\left(s, x, y_{0}\right)>0$ for some $s>0$ ) and $\operatorname{PHI}(\phi)$ again, there are constants $s_{0}>0$ and $r_{1} \in\left(0, r_{0}\right]$ so that $p^{B}(t, x, z)>0$ for every $t \geq s_{0}$ and $z \in B\left(y_{0}, r_{1}\right) \backslash \mathcal{N}_{1}$. It follows then

$$
\psi(x)=e^{\lambda_{0} t} P_{t}^{B} \psi(x) \geq e^{\lambda_{0} t} \int_{B\left(y_{0}, r_{1}\right)} p^{B}(t, x, z) \psi(z) \mu(d z)>0 .
$$

The claim that $\psi>0$ on $\widehat{B}$ is proved. In particular, $\psi\left(\widehat{x}_{0}\right)>0$.

(iii) Let $C_{i}(i=1, \ldots, 6)$ be the constants in the definition of $\operatorname{PHI}(\phi)$. Applying $\operatorname{PHI}(\phi)$ to the function $v(t, x)=e^{-\lambda_{0} t} \psi(x)$ in the cylinder $Q\left(0, x_{0}, C_{4} \phi(r), r\right)$, we get that

$$
v\left(t_{-}, \widehat{x}_{0}\right) \leq C_{6} v\left(t_{+}, \widehat{x}_{0}\right),
$$

where $t_{-}=\frac{C_{1}+C_{2}}{2} \phi(r)$ and $t_{+}=\frac{C_{3}+C_{4}}{2} \phi(r)$. It follows from (3.9) that

$$
e^{-\lambda_{0} t_{-}} \psi\left(\widehat{x}_{0}\right) \leq C_{6} e^{-\lambda_{0} t_{+}} \psi\left(\widehat{x}_{0}\right)
$$

Since $\psi\left(\widehat{x}_{0}\right)>0$, we arrive at

$$
\lambda_{0} \leq \frac{\log C_{6}}{t_{+}-t_{-}} \leq \frac{1}{\phi(\kappa r)}
$$


where $\kappa>0$ is chosen so that

$$
\frac{\left(C_{3}+C_{4}\right)-\left(C_{1}+C_{2}\right)}{2} \phi(r / 2) \geq \phi(\kappa r) \log C_{6}
$$

for all $r>0$. This along with (3.8) further yields that for all $t>0$,

$$
\operatorname{ess} \sup _{x, y \in B} p^{B}(t, x, y) \geq \frac{1}{\mu(B)} e^{-\frac{t}{\phi(\kappa r)}} .
$$

Following the arguments between (4.52) and (4.60) in [BGK, 1130-1131] line by line with small modifications, we obtain that there is a constant $c^{\prime}>0$ such that for all $x$, $y \in B\left(x_{0}, C_{5} r\right) \backslash \mathcal{N}_{1}$ and $t \in\left(t_{0}+C_{3} \phi(r), t_{0}+C_{4} \phi(r)\right)$ with $t_{0}=\left(C_{3}-C_{1}\right) \phi(r)$,

$$
p^{B}(t, x, y) \geq \frac{c^{\prime}}{V\left(x_{0}, r\right)} .
$$

Note that, in order to get (3.10) we should change [BGK, (4.57)] into

$$
\operatorname{ess}_{\sup _{x \in B^{\prime}}} p^{B}(s, x, z) \leq C_{6} p^{B}(t, y, z), \quad y, z \in B^{\prime}:=B\left(x_{0}, C_{5} r\right) \backslash \mathcal{N}_{1} .
$$

Furthermore, using (3.10) instead of [BGK, (4.60)], one can verify that NDL $(\phi)$ holds for this case by the almost same argument between (4.60) and (4.63) in [BGK, 1131-1132].

We next prove that $\operatorname{PHI}(\phi)$ implies UJS.

Proposition 3.3. Under VD and (1.11), $\mathrm{PHI}(\phi)$ implies UJS.

Proof. (i) Since $(\mathcal{E}, \mathcal{F})$ is a regular Dirichlet form on $L^{2}(M ; \mu)$, for any relatively compact open sets $U$ and $V$ with $\bar{U} \subset V$, there is a function $\psi \in \mathcal{F} \cap C_{c}(M)$ such that $\psi=1$ on $U$ and $\psi=0$ on $V^{c}$. Consequently,

$$
\int_{U \times V^{c}} J(d x, d y)=\int_{U \times V^{c}}(\psi(x)-\psi(y))^{2} J(d x, d y) \leq \mathcal{E}(\psi, \psi)<\infty .
$$

Since $U$ and $V$ are arbitrarily, we get that for almost all $x \in M$ and each $r>0$,

$$
J\left(x, B(x, r)^{c}\right)<\infty .
$$

(ii) Let $D$ be an open set of $M$, and $f(t, z)$ be a bounded and non-negative function on $(0, \infty) \times D^{c}$. Then

$$
u(t, z):= \begin{cases}\mathbb{E}^{z}\left[f\left(t-\tau_{D}, X_{\tau_{D}}\right) ; \tau_{D} \leq t\right], & t>0, z \in M_{0}, \\ 0, & t>0, z \in \mathcal{N}\end{cases}
$$

is non-negative on $(0, \infty) \times M$ and caloric in $(0, \infty) \times D$. In the proof below, the constants $C_{i}(i=1, \ldots, 6)$ are taken from the definition of $\operatorname{PHI}(\phi)$. For any $x, y \in M_{0}$ and $0<r \leq$ $\frac{1}{2} d(x, y)$. For any $0<\varepsilon<r$ and $0<h<\left(C_{1}+C_{2}\right) \phi(r) / 2$, define

$$
f_{h}(t, z)=\mathbf{1}_{\left(\left(C_{1}+C_{2}\right) \phi(r) / 2-h,\left(C_{1}+C_{2}\right) \phi(r) / 2\right)}(t) \mathbf{1}_{B(y, \varepsilon)}(z), \quad t>0, z \in M .
$$


For $t \geq\left(C_{1}+C_{2}\right) \phi(r) / 2$, define

$$
\begin{aligned}
u_{h}(t, z) & =\mathbb{E}^{z}\left[f_{h}\left(t-\tau_{B(x, r)}, X_{\tau_{B(x, r)}}\right) ; \tau_{B(x, r)} \leq t\right] \\
& =\mathbb{P}^{z}\left(X_{\tau_{B(x, r)}} \in B(y, \varepsilon), t-\left(C_{1}+C_{2}\right) \phi(r) / 2<\tau_{B(x, r)}<t-\left(C_{1}+C_{2}\right) \phi(r) / 2+h\right)
\end{aligned}
$$

if $z \in M_{0}$, and $u_{h}(t, z)=0$ if $z \in \mathcal{N}$.

According to Lemma 2.11, for any $z \in B(x, r) \cap M_{0}$ and $t \geq\left(C_{1}+C_{2}\right) \phi(r) / 2$,

$$
\begin{aligned}
u_{h}(t, z) & =\mathbb{E}^{z}\left[\int_{0}^{\tau_{B(x, r)}} d v \int_{B(y, \varepsilon)} \mathbf{1}_{\left(t-\left(C_{1}+C_{2}\right) \phi(r) / 2, t-\left(C_{1}+C_{2}\right) \phi(r) / 2+h\right)}(v) J\left(X_{v}, d u\right)\right] \\
& =\int_{t-\left(C_{1}+C_{2}\right) \phi(r) / 2}^{t-\left(C_{1}+C_{2}\right) \phi(r) / 2+h} \mathbb{E}^{z}\left[\mathbf{1}_{\left(0, \tau_{B(x, r)}\right)}(v) \int_{B(y, \varepsilon)} J\left(X_{v}, d u\right)\right] d v \\
& =\int_{t-\left(C_{1}+C_{2}\right) \phi(r) / 2}^{t-\left(C_{1}+C_{2}\right) \phi(r) / 2+h} P_{v}^{B(x, r)} H(z) d v,
\end{aligned}
$$

where $H(z):=\int_{B(y, \varepsilon)} J(z, d u)$.

Applying $\operatorname{PHI}(\phi)$ to $u_{h}$ in $Q\left(0, x, C_{4} \phi(r), r\right)$, we obtain that for any $x_{0} \in B\left(x, \varepsilon_{1}\right) \backslash\left(\mathcal{N}_{u_{h}} \cup\right.$ $\mathcal{N})$ with $\varepsilon_{1} \leq C_{5} r$

$$
u_{h}\left(\left(C_{1}+C_{2}\right) \phi(r) / 2, x_{0}\right) \leq C_{6} u_{h}\left(\left(C_{3}+C_{4}\right) \phi(r) / 2, x\right) .
$$

Now, by the definition of $u_{h}$ and Proposition 3.1.

$$
\begin{aligned}
u_{h}\left(\left(C_{3}+C_{4}\right) \phi(r) / 2, x\right) & =\int_{B(x, r)} p^{B(x, r)}\left(\frac{\left(C_{3}+C_{4}\right)-\left(C_{1}+C_{2}\right)}{2} \phi(r), x, z\right) \\
& \times u_{h}\left(\left(C_{1}+C_{2}\right) \phi(r) / 2, z\right) \mu(d z) \\
\leq & \frac{c_{1}}{V(x, r)} \int_{B(x, r)} u_{h}\left(\left(C_{1}+C_{2}\right) \phi(r) / 2, z\right) \mu(d z) .
\end{aligned}
$$

Combining both inequalities above and integrating by $\frac{1}{V\left(x, \varepsilon_{1}\right)} \int_{B\left(x, \varepsilon_{1}\right)} \cdots \mu\left(d x_{0}\right)$, we have

$$
\begin{aligned}
& \frac{1}{V\left(x, \varepsilon_{1}\right)} \int_{B\left(x, \varepsilon_{1}\right)} u_{h}\left(\left(C_{1}+C_{2}\right) \phi(r) / 2, x_{0}\right) \mu\left(d x_{0}\right) \\
& \leq \frac{c_{2}}{V(x, r)} \int_{B(x, r)} u_{h}\left(\left(C_{1}+C_{2}\right) \phi(r) / 2, z\right) \mu(d z) .
\end{aligned}
$$

According to (3.11), $H \in L^{1}(B(x, r) ; \mu)$. Then, as $h \rightarrow 0$,

$$
\begin{aligned}
\mid \int_{B\left(x, \varepsilon_{1}\right)} & \left(\frac{1}{h} u_{h}\left(\left(C_{1}+C_{2}\right) \phi(r) / 2, z\right)-H(z)\right) \mu(d z) \mid \\
\leq & \frac{1}{h} \int_{0}^{h} \int_{B\left(x, \varepsilon_{1}\right)}\left|P_{v}^{B(x, r)} H(z)-H(z)\right| \mu(d z) d v \\
\leq & \frac{1}{h} \int_{0}^{h}\left\|\left(P_{v}^{B(x, r)} H-H\right)\right\|_{L^{1}(B(x, r) ; \mu)} d v \rightarrow 0,
\end{aligned}
$$


thanks to the continuity of the semigroup $\left\{P_{t}^{B(x, r)}\right\}$ in $L^{1}(B(x, r) ; \mu)$. Similarly, we have

$$
\lim _{h \rightarrow 0}\left|\int_{B(x, r)}\left(\frac{1}{h} u_{h}\left(\left(C_{1}+C_{2}\right) \phi(r) / 2, z\right)-H(z)\right) \mu(d z)\right|=0
$$

Thus dividing both sides of (3.13) by $h$ and taking $h \rightarrow 0$, we have

$$
\frac{1}{V\left(x, \varepsilon_{1}\right)} \int_{B\left(x, \varepsilon_{1}\right)} \int_{B(y, \varepsilon)} J(z, d u) \mu(d z) \leq \frac{c_{2}}{V(x, r)} \int_{B(x, r)} \int_{B(y, \varepsilon)} J(z, d u) \mu(d z) .
$$

Letting $\varepsilon_{1} \rightarrow 0$, by (3.11), (3.12) and the Lebesgue differentiation theorem (e.g. see $\underline{\underline{H}}$, Theorem 1.8]), we find that for $\mu$-a.e $x \in M$,

$$
J(x, B(y, \varepsilon)) \leq \frac{c_{2}}{V(x, r)} \int_{B(x, r)} \int_{B(y, \varepsilon)} J(z, d u) \mu(d z)=\frac{c_{2}}{V(x, r)} \int_{B(y, \varepsilon)} \int_{B(x, r)} J(z, d u) \mu(d z) .
$$

The above inequality implies that $J(x, d y)$ is absolutely continuous with respect to the measure $\mu(d y)$. So there is a non-negative function $J(x, y)$ so that $J(x, d y)=J(x, y) \mu(d y)$. Since $J(d x, d y)$ is a symmetric measure, we may modify the values of $J(x, y)$ so that it is symmetric in $(x, y)$ for $\mu$-a.e. $x, y \in M$. Dividing the above by $V(y, \varepsilon)$ and then sending $\varepsilon \rightarrow 0$, we have by the Lebesgue differentiation theorem again that for $\mu$-a.e. $x, y \in M$ and $0<r<\frac{1}{2} d(x, y)$, we have

$$
J(x, y) \leq \frac{c_{2}}{V(x, r)} \int_{B(x, r)} J(z, y) \mu(d z)
$$

proving UJS.

Corollary 3.4. If VD, (1.11), UJS and $\mathrm{NDL}(\phi)$ are satisfied, then $\mathrm{J}_{\phi, \leq}$ holds. In particular, $\mathrm{J}_{\phi, \leq}$ holds under $\mathrm{VD}$, (1.11) and $\mathrm{PHI}(\phi)$.

Proof. For any $x \in M_{0}$ and $r, t>0$, by Lemma 2.11,

$$
\begin{aligned}
1 & \geq \mathbb{P}^{x}\left(X_{\tau_{B(x, r)}} \notin B(x, r), \tau_{B(x, r)} \leq t \text { and } \tau_{B(x, r)} \text { is a jumping time }\right) \\
& =\int_{0}^{t} \int_{B(x, r)} p^{B(x, r)}(s, x, y) J\left(y, B(x, r)^{c}\right) \mu(d y) d s .
\end{aligned}
$$

By using $\operatorname{NDL}(\phi)$ and taking $t=\phi(\varepsilon r)$ (where $\varepsilon \in(0,1)$ is the constant in the definition of $\operatorname{NDL}(\phi))$, we obtain that for any $x \in M_{0}$ and $r>0$,

$$
\begin{aligned}
1 & \geq \int_{t / 2}^{t} \int_{B\left(x, \varepsilon \phi^{-1}(t / 2)\right)} p^{B(x, r)}(s, x, y) J\left(y, B(x, r)^{c}\right) \mu(d y) d s \\
& \geq \frac{t}{2} \operatorname{essinf} \inf _{s \in[t / 2, t], y \in B\left(x, \varepsilon \phi^{-1}(t / 2)\right)} p^{B(x, r)}(s, x, y) \int_{B\left(x, \varepsilon \phi^{-1}(t / 2)\right)} J\left(y, B(x, r)^{c}\right) \mu(d y) \\
& \geq \frac{c_{1} t}{V\left(x, \phi^{-1}(t)\right)} \int_{B\left(x, \varepsilon \phi^{-1}(t / 2)\right)} J\left(y, B(x, r)^{c}\right) \mu(d y) .
\end{aligned}
$$


Thus, by VD and (1.11), there are constants $c_{2}, c_{3}>1$ such that

$$
\int_{B(x, r)} J\left(y, B\left(x, c_{2} r\right)^{c}\right) \mu(d y) \leq \frac{c_{3} V(x, r)}{\phi(r)} .
$$

For fixed $x, y \in M$, set $r=\frac{d(x, y)}{1+c_{2}} \leq \frac{d(x, y)}{2}$. Then, by (1.21) and (13.14),

$$
\begin{aligned}
J(x, y) & \leq \frac{c_{4}}{V(x, r)} \int_{B(x, r)} J(z, y) \mu(d z) \\
& \leq \frac{c_{4}^{2}}{V(x, r) V(y, r)} \int_{B(x, r)} \int_{B(y, r)} J(z, u) \mu(d u) \mu(d z) \\
& \leq \frac{c_{4}^{2}}{V(x, r) V(y, r)} \int_{B(x, r)} \int_{B\left(x, c_{2} r\right)^{c}} J(z, u) \mu(d u) \mu(d z) \\
& \leq \frac{c_{5}}{V(x, r) V(x, r)} \int_{B(x, r)} J\left(z, B\left(x, c_{2} r\right)^{c}\right) \mu(d z) \leq \frac{c_{6}}{V(x, r) \phi(r)}
\end{aligned}
$$

which completes the proof, thanks to VD and (1.11) again.

We note that by Proposition 3.1, Corollary [3.4, Proposition 3.5] in the next subsection and Theorem 1.12, we have $\operatorname{PHI}(\phi) \Longrightarrow \operatorname{UHK}(\phi)$.

\subsection{Consequences of $\operatorname{NDL}(\phi)$}

In this subsection, we present some consequences of $\operatorname{NDL}(\phi)$. Since $\operatorname{PHI}(\phi)$ implies $\operatorname{NDL}(\phi)$ by Proposition 3.2, this subsection can be regarded as a continuation of Subsection 3.1.

Proposition 3.5. Assume that VD, (1.11), and NDL $(\phi)$ hold. Then

(i) $\mathrm{PI}(\phi)$ holds. If furthermore RVD is satisfied, then $\mathrm{FK}(\phi)$ also holds.

(ii) $\mathrm{E}_{\phi, \geq}$ holds. If in addition RVD is satisfied, then we have $\mathrm{E}_{\phi, \leq}$ and so $\mathrm{E}_{\phi}$.

In particular, if $\mathrm{VD}, \mathrm{RVD},(1.11)$ and $\mathrm{PHI}(\phi)$ hold, then so do (i) and (ii).

Proof. (i) The main idea of the proof is due to [KS, Theorem 5.1], which is concerned with second order (degenerate) elliptic operators. See also the proof of [Sa2, Theorem 5.5.2] for related arguments. For any $x_{0} \in M$ and $r>0$, let $B=B\left(x_{0}, r\right)$. Define a bilinear form $(\overline{\mathcal{E}}, \overline{\mathcal{F}})$ on $L^{2}(B ; \mu)$ by

$$
\begin{aligned}
\overline{\mathcal{E}}(u, v) & =\int_{B \times B}(u(x)-u(y))(v(x)-v(y)) J(x, y) \mu(d x) \mu(d y), \\
\overline{\mathcal{F}} & =\left\{u \in L^{2}(B ; \mu): \overline{\mathcal{E}}(u, u)<\infty\right\} .
\end{aligned}
$$

One can easily check by using Fatou's lemma that $(\overline{\mathcal{E}}, \overline{\mathcal{F}})$ is closable and is a Dirichlet form on $L^{2}(B ; \mu)$. Let $\left\{\bar{P}_{t}\right\}$ be the $L^{2}$-semigroup associated with $(\overline{\mathcal{E}}, \overline{\mathcal{F}})$. Let $\overline{\mathcal{F}}_{B}$ be the closure of $\overline{\mathcal{F}} \cap C_{c}(B)$. Then $\left(\overline{\mathcal{E}}, \overline{\mathcal{F}}_{B}\right)$ is a regular Dirichlet form on $L^{2}(B ; \mu)$, whose associated 
semigroup will be denoted as $\left\{\bar{P}_{t}^{B}\right\}$. By [CF, Theorem 5.2.17], $\left(\overline{\mathcal{E}}, \overline{\mathcal{F}}_{B}\right)$ is the resurrected Dirichlet form of $\left(\mathcal{E}, \mathcal{F}_{B}\right)$. In other words, if we denote by $\bar{X}^{B}=\left\{\bar{X}_{t}^{B}\right\}$ the Hunt process associated with the regular Dirichlet form $\left(\overline{\mathcal{E}}, \overline{\mathcal{F}}_{B}\right)$ on $L^{2}(B ; \mu)$, then $\bar{X}^{B}$ is the resurrection of $X^{B}=\left\{X_{t}^{B}\right\}$ in $B$, and so $\bar{X}^{B}$ can be obtained from $X^{B}$ by creation through a FeynmanKac transform. Consequently, $\bar{X}^{B}$ has a transition density function $\bar{p}^{B}(t, x, y)$ with respect to $\mu$ and $\bar{p}^{B}(t, x, y) \geq p^{B}(t, x, y)$ for every $t>0$ and $x, y \in B \cap M_{0}$. This together with $\operatorname{NDL}(\phi)$ implies that there exist $\varepsilon \in(0,1)$ and $c_{1}>0$ such that for all $x_{0} \in M$ and $x$, $y \in B\left(x_{0}, \varepsilon^{2} r\right) \cap M_{0}$,

$$
\bar{p}^{B}(\phi(\varepsilon r), x, y) \geq p^{B}(\phi(\varepsilon r), x, y) \geq \frac{c_{1}}{V\left(x_{0}, r\right)}
$$

On the other hand, we know from [CF, Section 6.2], $(\overline{\mathcal{E}}, \overline{\mathcal{F}})$ is the active reflected Dirichlet space for $\left(\overline{\mathcal{E}}, \overline{\mathcal{F}}_{B}\right)$. Although $(\overline{\mathcal{E}}, \overline{\mathcal{F}})$ may not be regular as a Dirichlet form on $L^{2}(B ; \mu)$, by Silverstein [Si, Theorem 20.1], there is a locally compact separable metric space $\widetilde{B}$ (called regularizing space) so that $(\overline{\mathcal{E}}, \overline{\mathcal{F}})$ is regular on $L^{2}(\widetilde{B} ; \widetilde{\mu})$ and $B$ is intrinsically open in $\widetilde{B}$. Here $\widetilde{\mu}$ is an extension of $\mu$ to $\widetilde{B}$ by setting $\widetilde{\mu}(\widetilde{B} \backslash B)=0$. Let $\widetilde{X}=\left\{\widetilde{X}_{t}\right\}$ denote the Hunt process on $\widetilde{B}$ associated with the regular Dirichlet form $(\overline{\mathcal{E}}, \overline{\mathcal{F}})$ on $L^{2}(\widetilde{B} ; \mu)$. Then the part process $\widetilde{X}^{B}=\left\{\widetilde{X}_{t}^{B}\right\}$ of $\widetilde{X}$ killed upon leaving $B$ has the same distribution as $\bar{X}^{B}$. Now for $f \in \overline{\mathcal{F}}$, by the basic property of Dirichlet form (see, for example, [CF, (1.1.4)]),

$$
\begin{aligned}
\overline{\mathcal{E}}(f, f) & \geq \frac{1}{\phi(\varepsilon r)} \int_{B} f(x)\left(f-\bar{P}_{\phi(\varepsilon r)} f\right)(x) \mu(d x) \\
& \geq \frac{1}{2 \phi(\varepsilon r)} \mathbb{E}^{\widetilde{\mu}}\left[\left(f\left(\widetilde{X}_{\phi(\varepsilon r)}\right)-f\left(\widetilde{X}_{0}\right)\right)^{2}\right] \\
& \geq \frac{1}{2 \phi(\varepsilon r)} \mathbb{E}^{\widetilde{\mu}}\left[\left(f\left(\widetilde{X}_{\phi(\varepsilon r)}\right)-f\left(\widetilde{X}_{0}\right)\right)^{2} ; \phi(\varepsilon r)<\tau_{B}\right] \\
& =\frac{1}{2 \phi(\varepsilon r)} \int_{B \times B} \bar{p}^{B}(\phi(\varepsilon r), x, y)(f(x)-f(y))^{2} \mu(d x) \mu(d y) \\
& \geq \frac{c_{2}}{V\left(x_{0}, r\right) \phi(r)} \int_{B\left(x_{0}, \varepsilon^{2} r\right)} \int_{B\left(x_{0}, \varepsilon^{2} r\right)}(f(x)-f(y))^{2} \mu(d x) \mu(d y) \\
& \geq \frac{c_{3}}{\phi(r)} \int_{B\left(x_{0}, \varepsilon^{2} r\right)}\left(f(x)-\bar{f}_{B\left(x_{0}, \varepsilon^{2} r\right)}\right)^{2} \mu(d x)
\end{aligned}
$$

Recall that $\bar{f}_{D}:=\frac{1}{\mu(D)} \int_{D} f d \mu$ for any open set $D$ of $M$. In the last two inequalities above we have used VD, (1.11) and the fact that

$$
\int_{B\left(x_{0}, \varepsilon^{2} r\right)}\left(f(x)-\bar{f}_{B\left(x_{0}, \varepsilon^{2} r\right)}\right)^{2} \mu(d x)=\inf _{a \in \mathbb{R}} \int_{B\left(x_{0}, \varepsilon^{2} r\right)}(f(x)-a)^{2} \mu(d x) .
$$

This establishes $\operatorname{PI}(\phi)$.

That $\operatorname{PI}(\phi)$ implies FK $(\phi)$ under additional assumption RVD is given in Proposition 2.9. (Note that, under additional assumption $\operatorname{RVD}, \mathrm{FK}(\phi)$ is also a direct consequence of $\mathrm{PHI}(\phi)$, thanks to Propositions 3.1 and 2.9.) 
(ii) By VD, (1.11) and $\operatorname{NDL}(\phi)$, for some $\varepsilon \in(0,1)$,

$$
\begin{aligned}
\mathbb{P}^{x}\left(\tau_{B(x, r)} \geq \phi(\varepsilon r)\right) & =\int_{B(x, r)} p^{B(x, r)}(\phi(\varepsilon r), x, y) \mu(d y) \\
& \geq \int_{B\left(x, \varepsilon^{2} r\right)} p^{B(x, r)}(\phi(\varepsilon r), x, y) \mu(d y) \geq c_{6},
\end{aligned}
$$

and thus $\mathbb{E}^{x_{0}} \tau_{B\left(x_{0}, r\right)} \geq c_{6} \phi(r)$. This proves $\mathrm{E}_{\phi, \geq}$.

Next, we assume that RVD is satisfied. Let $B=B\left(x_{0}, r\right)$ with $x_{0} \in M_{0}$ and $r>0$, and $B^{\prime}=B\left(x_{0}, r /\left(2 l_{\mu}\right)\right)$, where $l_{\mu}>1$ is the constant in (1.10). Then, VD, (1.11) and NDL $(\phi)$ give us that for $t=\phi(r / \varepsilon)$ with some $\varepsilon \in(0,1)$,

$$
p(t, x, y) \geq \frac{c_{1}}{V\left(x_{0}, r\right)}, \quad x, y \in B \backslash \mathcal{N} .
$$

Fix $y_{0} \in M$ with $\left(1+2 l_{\mu}\right) r /\left(2 l_{\mu}\left(1+l_{\mu}\right)\right)<d\left(x_{0}, y_{0}\right)<\left(1+2 l_{\mu}\right) r /\left(2\left(1+l_{\mu}\right)\right)$ (such a point $y_{0}$ indeed exists due to RVD), then for any $x \in B^{\prime} \backslash \mathcal{N}$,

$$
\begin{aligned}
\mathbb{P}^{x}\left(X_{t} \notin B^{\prime}\right) & \geq \mathbb{P}^{x}\left(X_{t} \in B\left(y_{0}, r /\left(2\left(1+l_{\mu}\right)\right)\right)\right)=\int_{B\left(y_{0}, r /\left(2\left(1+l_{\mu}\right)\right)\right)} p(t, x, y) \mu(d y) \\
& \geq \frac{c_{2} V\left(y_{0}, r /\left(2\left(1+l_{\mu}\right)\right)\right)}{V\left(x_{0}, r\right)} \geq c_{3},
\end{aligned}
$$

where VD is used in the last inequality. So, we have $\mathbb{P}^{x}\left(\tau_{B^{\prime}}>t\right) \leq \mathbb{P}^{x}\left(X_{t} \in B^{\prime}\right) \leq 1-c_{3}$ for all $x \in B^{\prime} \backslash \mathcal{N}$. Hence, by the Markov property, $\mathbb{P}^{x}\left(\tau_{B^{\prime}}>k t\right) \leq\left(1-c_{3}\right)^{k}$, and thus $\mathbb{E}^{x} \tau_{B^{\prime}} \leq c_{4} t$. Since $\mathbb{E}^{x_{0}} \tau_{B\left(x_{0}, r /\left(2 l_{\mu}\right)\right)}=\mathbb{E}^{x_{0}} \tau_{B^{\prime}}$, replacing $r /\left(2 l_{\mu}\right)$ by $r$ gives us that $\mathbb{E}^{x_{0}} \tau_{B\left(x_{0}, r\right)} \leq c_{5} \phi(r)$, where (1.11) is used in the inequality above. Therefore, $\mathrm{E}_{\phi}$ holds. (Note that, by Lemma 2.8, under $\mathrm{VD}$ and (1.11), $\mathrm{FK}(\phi)$ implies $\mathrm{E}_{\phi, \leq}$. Then, $\mathrm{E}_{\phi, \leq}$ can be also deduced from $\operatorname{PHI}(\phi)$ directly under additional assumption RVD, thanks to Propositions 3.1 and 2.9.)

Combining all the conclusions of this and previous subsections, we can obtain the following main result in this section.

Theorem 3.6. Assume that $\mu$ and $\phi$ satisfy VD, RVD and (1.11) respectively. Then the following hold

$$
\begin{aligned}
\operatorname{PHI}(\phi) & \Longleftrightarrow \mathrm{UHKD}(\phi)+\mathrm{NDL}(\phi)+\mathrm{UJS}+\mathrm{E}_{\phi}+\mathrm{J}_{\phi, \leq} \\
& \Longleftrightarrow \mathrm{UHKD}(\phi)+\mathrm{NDL}(\phi)+\mathrm{UJS} \\
& \Longleftrightarrow \mathrm{UHK}(\phi)+\mathrm{NDL}(\phi)+\mathrm{UJS} .
\end{aligned}
$$

Proof. Note that by Corollary [3.4, NDL $(\phi)+$ UJS $\Longrightarrow \mathrm{J}_{\phi, \leq}$; and that by Proposition 3.5, $\mathrm{NDL}(\phi)$ implies $\mathrm{E}_{\phi}$. According to Theorem 1.12, UHK $(\phi)+$ conservativeness $\Longleftrightarrow \mathrm{UHKD}(\phi)+\mathrm{J}_{\phi, \leq}+\mathrm{E}_{\phi}$. Then the required assertion now follows from all the previous propositions. (Here we note that both $\operatorname{PHI}(\phi)$ and $\operatorname{NDL}(\phi)$ imply the conservativeness of the process $\left\{X_{t}\right\}$, see Proposition 2.4 and Proposition 3.2.) 


\section{$3.3 \quad$ Hölder regularity}

Another consequence of $\operatorname{NDL}(\phi)$ is that, it along with $\mathrm{E}_{\phi, \leq}$ and $\mathrm{J}_{\phi, \leq}$ implies the joint Hölder regularity of bounded caloric functions. In other words, $\operatorname{NDL}(\phi)+\mathrm{E}_{\phi, \leq}+\mathrm{J}_{\phi, \leq}$ imply $\operatorname{PHR}(\phi)$ and EHR. For our purpose, in the following lemma we use the definition of $\operatorname{NDL}(\phi)$ with $\varepsilon \phi(r)$ and $\phi^{-1}(\varepsilon t)$ replaced by $\phi(\varepsilon r)$ and $\varepsilon \phi^{-1}(t)$, respectively.

Lemma 3.7. Suppose that $\mathrm{VD}$, (1.11) and $\mathrm{NDL}(\phi)$ hold. For every $0<\delta \leq \varepsilon$ (where $\varepsilon$ is the constant in the definition of $\mathrm{NDL}(\phi))$, there exists a constant $C_{1}>0$ such that for every $r>$ $0, x \in M_{0}, t \geq \delta \phi(r)$ and any compact set $A \subset[t-\delta \phi(r), t-\delta \phi(r) / 2] \times B\left(x, \phi^{-1}(\varepsilon \delta \phi(r) / 2)\right)$,

$$
\mathbb{P}^{(t, x)}\left(\sigma_{A}<\tau_{[t-\delta \phi(r), t] \times B(x, r)}\right) \geq C_{1} \frac{m \otimes \mu(A)}{V(x, r) \phi(r)},
$$

where $m \otimes \mu$ is a product of the Lebesgue measure on $\mathbb{R}_{+}$and $\mu$ on $M$.

Proof. The proof is almost the same as that for [CKK2, Lemma 4.9(i)]. Let $\tau_{r}=$ $\tau_{[t-\delta \phi(r), t] \times B(x, r)}$ and $A_{s}=\{y \in M:(s, y) \in A\}$. For any $t, r>0$ and $x \in M_{0}$,

$$
\begin{aligned}
\delta \phi(r) \mathbb{P}^{(t, x)}\left(\sigma_{A}<\tau_{r}\right) & \geq \int_{0}^{\delta \phi(r)} \mathbb{P}^{(t, x)}\left(\int_{0}^{\tau_{r}} \mathbf{1}_{A}\left(t-s, X_{s}\right) d s>0\right) d u \\
& \geq \int_{0}^{\delta \phi(r)} \mathbb{P}^{(t, x)}\left(\int_{0}^{\tau_{r}} \mathbf{1}_{A}\left(t-s, X_{s}\right) d s>u\right) d u \\
& =\mathbb{E}^{(t, x)}\left[\int_{0}^{\tau_{r}} \mathbf{1}_{A}\left(t-s, X_{s}\right) d s\right] .
\end{aligned}
$$

Note that, for any $t \geq \delta \phi(r)$,

$$
\begin{aligned}
\mathbb{E}^{(t, x)}\left[\int_{0}^{\tau_{r}} \mathbf{1}_{A}\left(t-s, X_{s}\right) d s\right] & =\int_{\delta \phi(r) / 2}^{\delta \phi(r)} \mathbb{P}^{(t, x)}\left(\left(t-s, X_{s}^{B(x, r)}\right) \in A\right) d s \\
& =\int_{\delta \phi(r) / 2}^{\delta \phi(r)} \mathbb{P}^{x}\left(X_{s}^{B(x, r)} \in A_{t-s}\right) d s \\
& =\int_{\delta \phi(r) / 2}^{\delta \phi(r)} d s \int_{A_{t-s}} p^{B(x, r)}(s, x, y) \mu(d y) .
\end{aligned}
$$

By VD, (1.11) and $\operatorname{NDL}(\phi)$, for any $s \in[\delta \phi(r) / 2, \delta \phi(r)]$ and $y \in B\left(x, \phi^{-1}(\varepsilon \delta \phi(r) / 2)\right) \backslash \mathcal{N}$,

$$
p^{B(x, r)}(s, x, y) \geq \frac{c_{1}}{V(x, r)} .
$$

Thus,

$$
\mathbb{E}^{(t, x)}\left[\int_{0}^{\tau_{r}} \mathbf{1}_{A}\left(t-s, X_{s}\right) d s\right] \geq \frac{c_{1}}{V(x, r)} \int_{\delta \phi(r) / 2}^{\delta \phi(r)} d s \int_{A_{t-s}} \mu(d y)=\frac{c_{1} m \otimes \mu(A)}{V(x, r)} .
$$

Combining all the conclusions above, we obtain the desired assertion. 
Proposition 3.8. Assume that VD, (1.11), $\mathrm{NDL}(\phi), \mathrm{E}_{\phi, \leq}$ and $\mathrm{J}_{\phi, \leq}$ hold. For every $\delta \in$ $(0,1)$, there exist positive constants $C>0$ and $\gamma \in(0,1]$, where $\gamma$ is independent of $\delta$, so that for any bounded caloric function $u$ in $Q\left(t_{0}, x_{0}, \phi(r), r\right)$, there is a properly exceptional set $\mathcal{N}_{u} \supset \mathcal{N}$ such that

$$
|u(s, x)-u(t, y)| \leq C\left(\frac{\phi^{-1}(|s-t|)+d(x, y)}{r}\right)^{\gamma} \operatorname{ess} \sup _{\left[t_{0}, t_{0}+\phi(r)\right] \times M}|u|
$$

for every $s, t \in\left(t_{0}+\phi(r)-\phi(\delta r), t_{0}+\phi(r)\right)$ and $x, y \in B\left(x_{0}, \delta r\right) \backslash \mathcal{N}_{u}$. In other words, under $\mathrm{VD}$ and (1.11), $\mathrm{NDL}(\phi)+\mathrm{E}_{\phi, \leq}+\mathrm{J}_{\phi, \leq}$ imply $\operatorname{PHR}(\phi)$ and $\mathrm{EHR}$.

Proof. With estimate (3.15), the result can be proved in exactly the same way as that for CK1, Theorem 4.14]. We omit the details here. We note that in the present paper the time evolves as $V_{s}=V_{0}-s$, which is opposed to $V_{s}=V_{0}+s$ as in [CK1, p. 37], so here we should reserve the time interval in the statement. (The statement of [CKK1, Theorem 3.1] should be corrected in the same way.)

The following consequence of Hölder regularities will be used in Subsection 4.2.

Lemma 3.9. Suppose EHR holds. Let $D \subset M$ be an open set with ess $\sup _{y \in D \cap M_{0}} \mathbb{E}^{y} \tau_{D}<\infty$. Fix a function $f \in B_{b}(D)$ and set $u=G^{D} f$. Then for any $B\left(x_{0}, r\right) \subset D$ and $0<r_{1} \leq r$,

$$
\operatorname{Osc}_{B\left(x_{0}, r_{1}\right) \cap M_{0}} u \leq 2 \sup _{y \in B\left(x_{0}, r\right) \cap M_{0}}|f(y)| \sup _{y \in B\left(x_{0}, r\right) \cap M_{0}} \mathbb{E}^{y} \tau_{B\left(x_{0}, r\right)}+c\left(r_{1} / r\right)^{\theta} \sup _{z \in D \cap M_{0}}|u(z)|,
$$

where $c>0$ and $\theta \in(0,1]$ only depend on the constants in EHR.

Proof. Note that for any $x \in D \cap M_{0}$,

$$
G^{D}|f|(x)=\mathbb{E}^{x}\left[\int_{0}^{\tau_{D}}\left|f\left(X_{t}\right)\right| d t\right] \leq \sup _{y \in D \cap M_{0}}|f(y)| \mathbb{E}^{x} \tau_{D}
$$

Consequently, for any $r_{1} \in(0, r)$,

$$
\begin{aligned}
\operatorname{Osc}_{B\left(x, r_{1}\right) \cap M_{0}} G^{B\left(x_{0}, r\right)} f & \leq 2 \sup _{y \in B\left(x_{0}, r_{1}\right) \cap M_{0}} G^{B\left(x_{0}, r\right)}|f|(y) \\
& \leq 2 \sup _{y \in B\left(x_{0}, r\right) \cap M_{0}}|f(y)| \sup _{y \in B\left(x_{0}, r_{1}\right) \cap M_{0}} \mathbb{E}^{y} \tau_{B(x, r)} .
\end{aligned}
$$

Since $G^{D} f(y)-G^{B\left(x_{0}, r\right)} f(y)=\mathbb{E}^{y}\left[G^{D} f\left(X_{\tau_{B\left(x_{0}, r\right)}}\right)\right]=\mathbb{E}^{y}\left[u\left(X_{\tau_{B\left(x_{0}, r\right)}}\right)\right]$ is harmonic in $B\left(x_{0}, r\right)$, and $u=0$ outside $D$, we have by EHR and Remark 1.16(ii) that

$$
\begin{aligned}
& \operatorname{OSc}_{B\left(x, r_{1}\right) \cap M_{0} u} u \\
& \leq \operatorname{osc}_{B\left(x_{0}, r_{1}\right) \cap M_{0}} G^{B\left(x_{0}, r\right)} f+\operatorname{osc}_{B\left(x_{0}, r_{1}\right) \cap M_{0}}\left(G^{D} f-G^{B\left(x_{0}, r\right)} f\right) \\
& \leq 2 \sup _{y \in B(x, r) \cap M_{0}}|f(y)| \sup _{y \in B\left(x_{0}, r_{1}\right) \cap M_{0}} \mathbb{E}^{y} \tau_{B\left(x_{0}, r\right)}+c\left(r_{1} / r\right)^{\theta} \sup _{y \in D \cap M_{0}}\left|\mathbb{E}^{y}\left[u\left(X_{\tau_{B(x, r)}}\right)\right]\right| \\
& \leq 2 \sup _{y \in B(x, r) \cap M_{0}}|f(y)| \sup _{y \in B(x, r) \cap M_{0}} \mathbb{E}^{y} \tau_{B(x, r)}+c\left(r_{1} / r\right)^{\theta} \sup _{z \in D \cap M_{0}}|u(z)| .
\end{aligned}
$$

This proves the lemma. 


\section{Equivalences of $\mathrm{PHI}(\phi)$}

We have already given some part of the proof of Theorem 1.20 in Section 3 , In this section, we will complete the proof. In Subsection 4 .1. we prove $(1) \Longleftrightarrow(2) \Longleftrightarrow(3) \Longleftrightarrow(4)$. $(1) \Longleftrightarrow(5) \Longleftrightarrow(6)$ will be proved in Subsection 4.2, and $(1) \Longleftrightarrow(7)$ in Subsection 4.3 .

\section{1 $\mathrm{PHI}(\phi) \Longleftrightarrow \mathrm{PHI}^{+}(\phi) \Longleftrightarrow \mathrm{UHK}(\phi)+\mathrm{NDL}(\phi)+\mathrm{UJS} \Longleftrightarrow \mathrm{NDL}(\phi)+$ UJS}

In this subsection, we will establish $(1) \Longleftrightarrow(2) \Longleftrightarrow(3) \Longleftrightarrow(4)$ in Theorem 1.20. Since $(1) \Longrightarrow(3)$ is already proved in Subsection 3.1. and (1) $\Longrightarrow(2)$ and (3) $\Longrightarrow(4)$ hold trivially, it remains to show that prove $(4) \Longrightarrow(3) \Longrightarrow(2)$.

Lemma 4.1. Assume that VD, (1.11), $\operatorname{UHK}(\phi), \operatorname{NDL}(\phi)$ and UJS. Let $\delta \leq \varepsilon$ (where $\varepsilon \in(0,1)$ is the constant in the definition of $\mathrm{NDL}(\phi))$, and $\theta \geq 1 / 2$. Let $0<\delta_{0}<\delta$ and $0<\delta_{1}<\delta_{2}<\delta_{3}<\delta_{4}$ such that $\left(\delta_{3}-\delta_{2}\right) \phi(r) \geq \phi\left(\delta_{0} r\right)$ and $\delta_{4} \phi(r) \leq \phi(\delta r)$ for all $r>0$. Set

$$
Q_{1}=\left(t_{0}, t_{0}+\delta_{4} \phi(r)\right) \times B\left(x_{0}, \delta_{0}^{2} r\right), \quad Q_{2}=\left(t_{0}, t_{0}+\delta_{4} \phi(r)\right) \times B\left(x_{0}, r\right)
$$

for $x_{0} \in M, t_{0} \geq 0$ and $r>0$. Define

$$
Q_{3}=\left[t_{0}+\delta_{1} \phi(r), t_{0}+\delta_{2} \phi(r)\right] \times B\left(x_{0}, \delta_{0}^{2} r / 2\right) \backslash \mathcal{N}
$$

and

$$
Q_{4}=\left[t_{0}+\delta_{3} \phi(r), t_{0}+\delta_{4} \phi(r)\right] \times B\left(x_{0}, \delta_{0}^{2} r / 2\right) \backslash \mathcal{N} .
$$

Let $f:\left(t_{0}, \infty\right) \times M \rightarrow \mathbb{R}_{+}$be bounded and supported in $\left(t_{0}, \infty\right) \times B\left(x_{0},(1+\theta) r\right)^{c}$. Then there is a constant $C_{2}>0$ such that the following holds:

$$
\mathbb{E}^{\left(t_{1}, y_{1}\right)} f\left(Z_{\tau_{Q_{1}}}\right) \leq C_{2} \mathbb{E}^{\left(t_{2}, y_{2}\right)} f\left(Z_{\tau_{Q_{2}}}\right) \quad \text { for every }\left(t_{1}, y_{1}\right) \in Q_{3} \text { and }\left(t_{2}, y_{2}\right) \in Q_{4} \text {. }
$$

Proof. The proof is the same as that of [CKK1, Lemma 5.3]. We present the proof here for the sake of completeness.

Without loss of generality, we may and do assume that $t_{0}=0$. For $x_{0} \in M$ and $s>0$, set $B_{s}=B\left(x_{0}, s\right)$. By Lemma 2.11, for any $\left(t_{2}, y_{2}\right) \in Q_{4}$,

$$
\begin{aligned}
& \mathbb{E}^{\left(t_{2}, y_{2}\right)} f\left(Z_{\tau_{Q_{2}}}\right)=\mathbb{E}^{\left(t_{2}, y_{2}\right)} f\left(t_{2}-\left(\tau_{B_{r}} \wedge t_{2}\right), X_{\tau_{B_{r}} \wedge t_{2}}\right) \\
& =\mathbb{E}^{\left(t_{2}, y_{2}\right)}\left[\int_{0}^{t_{2}} \mathbf{1}_{\left\{t \leq \tau_{B_{r}}\right\}} d t \int_{B_{(1+\theta) r}^{c}} f\left(t_{2}-t, v\right) J\left(X_{t}, v\right) \mu(d v)\right] \\
& =\int_{0}^{t_{2}} d t \int_{B_{(1+\theta) r}^{c}} f\left(t_{2}-t, v\right) \mathbb{E}^{\left(t_{2}, y_{2}\right)}\left[\mathbf{1}_{\left\{t \leq \tau_{B_{r}}\right\}} J\left(X_{t}, v\right)\right] \mu(d v) \\
& =\int_{0}^{t_{2}} d s \int_{B_{(1+\theta) r}^{c}} f(s, v) \mathbb{E}^{\left(t_{2}, y_{2}\right)}\left[\mathbf{1}_{\left\{t_{2}-s \leq \tau_{B_{r}}\right\}} J\left(X_{t_{2}-s}, v\right)\right] \mu(d v) \\
& =\int_{0}^{t_{2}} d s \int_{B_{(1+\theta) r}^{c}} f(s, v) \mu(d v) \int_{B_{r}} p^{B_{r}}\left(t_{2}-s, y_{2}, z\right) J(z, v) \mu(d z)
\end{aligned}
$$




$$
\geq \int_{0}^{t_{1}} d s \int_{B_{(1+\theta) r}^{c}} f(s, v) \mu(d v) \int_{B_{\delta_{0}^{2} r}} p^{B_{r}}\left(t_{2}-s, y_{2}, z\right) J(z, v) \mu(d z) .
$$

Since for $s \in\left[0, t_{1}\right], \phi\left(\delta_{0} r\right) \leq t_{2}-t_{1} \leq t_{2}-s \leq \phi(\delta r)$, by VD, (1.11) and NDL $(\phi)$, we know that the right hand side of the inequality above is greater than or equal to

$$
\frac{c_{1}}{V\left(x_{0}, r\right)} \int_{0}^{t_{1}} d s \int_{B_{(1+\theta) r}^{c}} f(s, v) \mu(d v) \int_{B_{\delta_{0}^{2} r}} J(z, v) \mu(d z) .
$$

So the proof is complete, once we can obtain that for every $\left(t_{1}, y_{1}\right) \in Q_{3}$,

$$
\mathbb{E}^{\left(t_{1}, y_{1}\right)} f\left(Z_{\tau_{Q_{1}}}\right) \leq \frac{c_{2}}{V\left(x_{0}, r\right)} \int_{0}^{t_{1}} d s \int_{B_{(1+\theta) r}^{c}} f(s, v) \mu(d v) \int_{B_{\delta_{0}^{2} r}} J(z, v) \mu(d z) .
$$

Similar to the argument for (4.1), we have by using Lemma 2.11,

$$
\begin{aligned}
\mathbb{E}^{\left(t_{1}, y_{1}\right)} f\left(Z_{\tau_{Q_{1}}}\right) & =\int_{0}^{t_{1}} d s \int_{B_{(1+\theta) r}^{c}} f(s, v) \mu(d v) \int_{B_{\delta_{0}^{2} r}} p^{B_{\delta_{0}^{2} r}\left(t_{1}-s, y_{1}, z\right) J(z, v) \mu(d z)} \\
& =\int_{0}^{t_{1}} d s \int_{B_{\delta_{0}^{2} r}} p^{B_{\delta_{0}^{2} r}\left(t_{1}-s, y_{1}, z\right) \mu(d z) \int_{B_{(1+\theta) r}^{c}} f(s, v) J(z, v) \mu(d v) .}
\end{aligned}
$$

Notice that

$$
\begin{aligned}
& \int_{B_{\delta_{0}^{2} r}} p^{B_{\delta_{0}^{2} r}\left(t_{1}-s, y_{1}, z\right) \mu(d z)} \int_{B_{(1+\theta) r}^{c}} f(s, v) J(z, v) \mu(d v) \\
& =\int_{B_{\delta_{0}^{2} r} \backslash B_{3 \delta_{0}^{2} r / 4}} p^{B_{\delta_{0}^{2}}^{2}\left(t_{1}-s, y_{1}, z\right) \mu(d z)} \int_{B_{(1+\theta) r}^{c}} f(s, v) J(z, v) \mu(d v)
\end{aligned}
$$

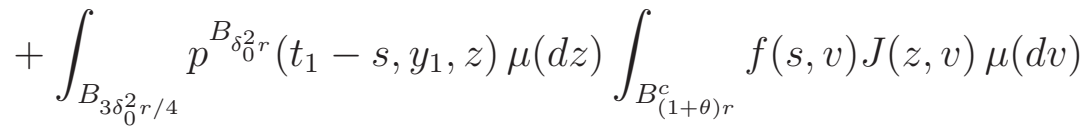

$$
\begin{aligned}
& =: I_{1}+I_{2} \text {. }
\end{aligned}
$$

On the one hand, when $z \in\left(B_{\delta_{0}^{2} r} \backslash B_{3 \delta_{0}^{2} r / 4}\right) \cap M_{0}$, we have $\delta_{0}^{2} r / 4 \leq d\left(y_{1}, z\right) \leq 3 \delta_{0}^{2} r / 2$, and so by $\operatorname{UHK}(\phi), \mathrm{VD}$ and (1.11),

$$
p^{B_{\delta_{0}^{2}} r}\left(t_{1}-s, y_{1}, z\right) \leq \frac{c_{3} t_{1}}{V\left(y_{1}, d\left(y_{1}, z\right)\right) \phi\left(d\left(y_{1}, z\right)\right)} \leq \frac{c_{4}}{V\left(x_{0}, r\right)}
$$

for some constants $c_{3}, c_{4}>0$. Hence, $\int_{0}^{t_{1}} I_{1} d s$ is less than or equal to the right hand side of (4.2). On the other hand, for $z \in B_{3 \delta_{0}^{2} r / 4}$, by UJS and VD,

$$
\begin{aligned}
\int_{B_{(1+\theta) r}^{c}} J(z, v) f(s, v) \mu(d v) & \leq \frac{c_{5}}{V\left(x_{0}, r\right)} \int_{B\left(z, \delta_{0}^{2} r / 4\right)} J(w, v) \mu(d w) \int_{B_{(1+\theta) r}^{c}} f(s, v) \mu(d v) \\
& \leq \frac{c_{5}}{V\left(x_{0}, r\right)} \int_{B_{\delta_{0}^{2} r}} J(w, v) \mu(d w) \int_{B_{(1+\theta) r}^{c}} f(s, v) \mu(d v) .
\end{aligned}
$$


Note that the right hand side of the above inequality does not depend on $z$. Multiplying both sides by $p^{B_{\delta_{0}^{2}}^{2}}\left(t_{1}-s, y_{1}, z\right)$ and integrating over $z \in B_{3 \delta_{0}^{2} r / 4}$ and then over $s \in\left[0, t_{1}\right]$, we obtain that $\int_{0}^{t_{1}} I_{2} d s$ is also less than or equal to the right hand side of (4.2). This proves the lemma.

Once again, in the following lemma we use the definition of $\operatorname{NDL}(\phi)$ with $\varepsilon \phi(r)$ and $\phi^{-1}(\varepsilon t)$ replaced by $\phi(\varepsilon r)$ and $\varepsilon \phi^{-1}(t)$, respectively.

Lemma 4.2. Suppose that VD, (1.11) and $\operatorname{NDL}(\phi)$ hold. Let $0<\delta \leq \varepsilon / 4$ such that $4 \delta \phi(2 r) \leq \varepsilon \phi(r)$ for all $r>0$, where $\varepsilon \in(0,1)$ is the constant in the definition of $\operatorname{NDL}(\phi)$. Then there exists a constant $C_{3}>0$ such that for every $R>0, r \in\left(0, \phi^{-1}(\varepsilon \delta \phi(R) / 2) / 2\right]$, $x_{0} \in M, \delta \phi(R) / 2 \leq t-s \leq 4 \delta \phi(2 R), x \in B\left(x_{0}, \phi^{-1}(\varepsilon \delta \phi(R) / 2) / 2\right) \backslash \mathcal{N}$, and $z \in B\left(x_{0}\right.$, $\left.\phi^{-1}(\varepsilon \delta \phi(R) / 2)\right) \backslash \mathcal{N}$,

$$
\mathbb{P}^{(t, z)}\left(\sigma_{U(s, x, r)} \leq \tau_{[s, t] \times B\left(x_{0}, R\right)}\right) \geq C_{3} \frac{V(x, r)}{V(x, R)},
$$

where $U(s, x, r)=\{s\} \times B(x, r)$.

Proof. The left hand side of the desired estimate is equal to

$$
\mathbb{P}^{z}\left(X_{t-s}^{B\left(x_{0}, R\right)} \in B(x, r)\right)=\int_{B(x, r)} p^{B\left(x_{0}, R\right)}(t-s, z, y) \mu(d y) .
$$

By VD, (1.11), NDL $(\phi)$, and the facts that $\delta \phi(R) / 2 \leq t-s \leq 4 \delta \phi(2 R)$ and $B(x, r) \subset$ $B\left(x_{0}, \phi^{-1}(\varepsilon \delta \phi(R) / 2)\right)$, (4.3) is greater than or equal to

$$
c_{1} \frac{V(x, r)}{V(z, R)} \geq c_{2} \frac{V(x, r)}{V(x, R)} .
$$

This proves the desired assertion.

Having these two lemmas as well as Lemma 3.7 at hand, one can obtain the following form of $\mathrm{PHI}^{+}(\phi)$.

Theorem 4.3. Suppose that VD and (1.11) hold. Under $\operatorname{UHK}(\phi), \mathrm{NDL}(\phi)$ and UJS, the following $\mathrm{PHI}^{+}(\phi)$ holds: there exist constants $\delta>0, C>1$ and $K \geq 1$ such that for every $x_{0} \in M \backslash \mathcal{N}, t_{0} \geq 0, R>0$ and every non-negative function $u$ on $[0, \infty) \times M$ that is caloric on $Q:=\left(t_{0}, t_{0}+4 \delta \phi(C R)\right) \times B\left(x_{0}, C R\right)$, we have

$$
\operatorname{ess} \sup _{\left(t_{1}, y_{1}\right) \in Q_{-}} u\left(t_{1}, y_{1}\right) \leq K \operatorname{ess} \inf _{\left(t_{2}, y_{2}\right) \in Q_{+}} u\left(t_{2}, y_{2}\right),
$$

where $Q_{-}=\left[t_{0}+\delta \phi(C R), t_{0}+2 \delta \phi(C R)\right] \times B\left(x_{0}, R\right)$ and $Q_{+}=\left[t_{0}+3 \delta \phi(C R), t_{0}+4 \delta \phi(C R)\right) \times$ $B\left(x_{0}, R\right)$.

Proof. Let $\varepsilon \in(0,1)$ be the constant in $\operatorname{NDL}(\phi)$. Take and fix some $\delta \in(0, \varepsilon / 4]$ so that $\delta \phi(2 r) \leq \phi(\varepsilon r) / 4$ for all $r>0$ and take $\delta_{0} \in(0, \delta)$ so that $\phi\left(\delta_{0} r\right) \leq \delta \phi(r)$ for all $r>0$. The existence of such $\delta$ and $\delta_{0}$ is guaranteed by the assumption (1.11). We choose $\delta$ and $\delta_{0}$ in 
such a way so that Lemma 4.1 holds by taking $\delta_{j}$ to be $j \delta$ for $j=1,2,3,4$ there. Condition (1.11) ensures that there is a constant $c_{0} \in(0,1 / 2)$ so that $\phi^{-1}(\delta \varepsilon \phi(r) / 2) \geq c_{0} r$ for every $r>0$. Take

$$
C=\left(2 / c_{0}\right)+2 \text { and } C_{0}=C-2=2 / c_{0} .
$$

The reason of defining such $C_{0}$ is that the conclusion of Lemma 4.2 holds for any $x, z \in$ $B\left(x_{0}, R / C_{0}\right)$.

Let $u$ be a non-negative function on $[0, \infty) \times M$ that is caloric on $Q:=\left(t_{0}, t_{0}+4 \delta \phi(C R)\right) \times$ $B\left(x_{0}, C R\right)$. We will show (4.4) holds.

The proof below is mainly based on that of [CKK1, Theorem 5.2] with some non-trivial modifications; see also the proof of [CK1, Proposition 4.3] or of [CK2, Theorem 4.12], whose idea is originally due to BL2, Theorem 3.1]. Truncating $u$ by $n$ outside $Q$ and then passing $n \rightarrow \infty$ if needed, without loss of generality, we may and do assume that $t_{0}=0$, and that the function $u$ is bounded on $Q$, see Step 3 in the proof of [CKK1, Theorem 5.2] (e.g. page 1085 in CKK1]). Furthermore, by looking at $a u+b$ for suitable constants $a$ and $b$, we may and do assume that $\inf _{(t, y) \in Q_{+}} u(t, y)=1 / 2$. Let $\left(t_{*}, y_{*}\right) \in Q_{+}$be such that $u\left(t_{*}, y_{*}\right) \leq 1$. It is enough to show that $u(t, x)$ is bounded from above in $Q_{-}$by a constant that is independent of the function $u$.

For any $t \geq \delta \phi(r)$, set $Q^{\downarrow}(t, \delta, x, r)=[t-\delta \phi(r), t] \times B(x, r)$. Note that

$$
m \otimes \mu\left(Q^{\downarrow}(t, \delta, x, r)\right)=\delta \phi(r) V(x, r) .
$$

By Lemma 3.7, there exists a constant $c_{1} \in(0,1 / 2)$ so that for any $r \leq R / 2$ and any compact set $D$ satisfying that

$$
D \subset\left[t-\delta \phi(r), t-\frac{1}{2} \delta \phi(r)\right] \times B\left(x, c_{0} r\right) \subset Q^{\downarrow}(t, \delta, x, r)
$$

and

$$
m \otimes \mu(D) / m \otimes \mu\left(Q^{\downarrow}(t, \delta, x, r)\right) \geq \frac{c_{0}^{d_{2}}}{4 \widetilde{C}_{\mu}},
$$

we have

$$
\mathbb{P}^{(t, x)}\left(\sigma_{D}<\tau_{Q \downarrow(t, \delta, x, r)}\right) \geq c_{1},
$$

where $\widetilde{C}_{\mu}$ and $d_{2}$ are the constants in (3.1). Let $C_{2}$ be the constant $C_{2}$ in Lemma 4.1 with $\delta_{j}=j \delta$ and $\theta=1 / 2$. Define

$$
\eta=\frac{c_{1}}{3}, \quad \xi=\frac{1}{3} \wedge\left(C_{2}^{-1} \eta\right)
$$

We claim that there is a universal constant $K \geq 2$ to be determined later, which is independent of $R$ and the function $u$, such that $u \leq K$ on $Q_{-}$. We are going to prove this by contradiction.

Suppose this is not true. Then there is some point $\left(t_{1}, x_{1}\right) \in Q_{-}$such that $u\left(t_{1}, x_{1}\right) \geq$ $K$. We will show that there are a constant $\beta>0$ and a sequence of points $\left\{\left(t_{k}, x_{k}\right)\right\}$ in $\left[t_{0}+\delta \phi(C R) / 2, t_{0}+2 \delta \phi(C R)\right) \times B\left(x_{0}, 2 R\right) \subset Q$ so that $u\left(t_{k}, x_{k}\right) \geq(1+\beta)^{k-1} K$, which contradicts to the assumption that $u$ is bounded on $Q$. 
Recall that $\beta_{1}, \beta_{2}, c_{3}$ and $c_{4}$ are the constants in (1.11). Then, by (3.1) and (1.11), we have for all $x \in M$ and all $0<r_{1}<r_{2} \wedge r_{3}<\infty$ :

$$
\frac{V\left(x, r_{1}\right) \phi\left(r_{1}\right)}{V\left(x, r_{2}\right) \phi\left(r_{3}\right)} \geq \frac{1}{c_{4} \widetilde{C}_{\mu}}\left(\frac{r_{1}}{r_{2}}\right)^{d_{2}}\left(\frac{r_{1}}{r_{3}}\right)^{\beta_{2}} \text {. }
$$

Let $C_{3}$ be the constant in Lemma 4.2, and set $r:=R K^{-1 /\left(2\left(d_{2}+\beta_{2}\right)\right)}$. We take $K \geq 2$ large enough so that $K \geq\left(2 \widetilde{C}_{\mu} /\left(C_{3} \xi \delta_{0}^{2 d_{2}}\right)\right)^{2}\left(2 C_{0}\right)^{d_{2}}$ and that, in view of (1.11),

$$
r<R / 8 \text { and } \phi(r)<\frac{1}{8} \phi(R) \quad \text { for all } R>0 \text { and } r=R K^{-1 /\left(2\left(d_{2}+\beta_{2}\right)\right)} .
$$

With such $r$, we have by (4.6)

$$
\frac{m \otimes \mu\left(Q^{\downarrow}(t, \delta, x, r)\right)}{\phi(R) V\left(x, C_{0} R\right)}=\frac{\delta \phi(r) V(x, r)}{\phi(R) V\left(x, C_{0} R\right)} \geq \frac{\delta}{c_{4} \widetilde{C}_{\mu} C_{0}^{d_{2}} \sqrt{K}} .
$$

Take $\tilde{t}=t_{1}+(5 / 2) \delta \phi(r)$ and define $\tilde{U}=\{\tilde{t}\} \times B\left(x_{1}, \delta_{0}^{2} r / 2\right)$. Observe that $t_{*}-\tilde{t} \geq \frac{1}{2} \delta \phi(C R)$ since $t_{*}-t_{1} \geq \delta \phi(C R)$. If the caloric function $u \geq \xi K$ on $\tilde{U}$, we would have by (4.5) and Lemma 4.2 that

$$
\begin{aligned}
1 \geq u\left(t_{*}, y_{*}\right) & =\mathbb{E}^{\left(t_{*}, y_{*}\right)} u\left(Z_{\sigma_{\tilde{U}} \wedge \tau_{Q_{*}}}\right) \geq \xi K \mathbb{P}^{\left(t_{*}, y_{*}\right)}\left(\sigma_{\tilde{U}} \leq \tau_{Q_{*}}\right) \geq \xi K \frac{C_{3} V\left(x_{1}, \delta_{0}^{2} r / 2\right)}{V\left(x_{1}, C_{0} R\right)} \\
& \geq \frac{C_{3} \xi K}{\widetilde{C}_{\mu}}\left(\delta_{0}^{2} r /\left(2 C_{0} R\right)\right)^{d_{2}} \geq \frac{C_{3} \xi \delta_{0}^{2 d_{2}} \sqrt{K}}{\left(2 C_{0}\right)^{d_{2}} \widetilde{C}_{\mu}} \geq 2,
\end{aligned}
$$

where $Q_{*}=\left[t_{1}-\delta \phi(r), t_{*}\right] \times B\left(x_{0}, C_{0} R\right)$. This contradiction yields that

$$
\text { there is some } y_{1} \in B\left(x_{1}, \delta_{0}^{2} r / 2\right) \text { so that } u\left(\tilde{t}, y_{1}\right)<\xi K \text {. }
$$

We next show that

$$
\mathbb{E}^{\left(t_{1}, x_{1}\right)}\left[u\left(Z_{\tau_{r}}\right): X_{\tau_{r}} \notin B\left(x_{1}, 3 r / 2\right)\right] \leq \eta K,
$$

where $\tau_{r}:=\tau_{\left(t_{1}-\delta \phi(r), t_{1}+3 \delta \phi(r)\right) \times B\left(x_{1}, \delta_{0}^{2} r\right)}$. If it is not true, then we would have by Lemma 4.1 with $\delta_{j}=j \delta(j=1,2,3,4)$ and $\theta=1 / 2$ that

$$
\begin{aligned}
\xi K>u\left(\tilde{t}, y_{1}\right) & \geq \mathbb{E}^{\left(\tilde{t}, y_{1}\right)}\left[u\left(Z_{\tau_{\left[t_{1}-\delta \phi(r), t_{1}+3 \delta \phi(r)\right] \times B\left(x_{1}, r\right)}}\right): X_{\tau_{\left[t_{1}-\delta \phi(r), t_{1}+3 \delta \phi(r)\right] \times B\left(x_{1}, r\right)}} \notin B\left(x_{1}, 3 r / 2\right)\right] \\
& \left.\geq C_{2}^{-1} \mathbb{E}^{\left(t_{1}, x_{1}\right)}\left[u\left(Z_{\tau_{r}}\right): X_{\tau_{r}} \notin B\left(x_{1}, 3 r / 2\right)\right)\right] \\
& >C_{2}^{-1} \eta K \geq \xi K,
\end{aligned}
$$

which is a contradiction. This establishes (4.8).

Let $A$ be any compact subset of

$$
\tilde{A}:=\left\{(s, y) \in\left[t_{1}-\delta \phi(r), t_{1}-\frac{1}{2} \delta \phi(r)\right] \times B\left(x_{1}, c_{0} r\right): u(s, y) \geq \xi K\right\},
$$


and define $U_{1}=\left\{t_{1}\right\} \times B\left(x_{1}, \delta_{0}^{2} r\right)$. By Lemmas 3.7 and 4.2 and the strong Markov property,

$$
\begin{aligned}
1 & \geq u\left(t_{*}, y_{*}\right) \geq \mathbb{E}^{\left(t_{*}, y_{*}\right)}\left[u\left(Z_{\sigma_{A}}\right): \sigma_{A} \leq \tau_{Q_{*}}\right] \\
& \geq \mathbb{E}^{\left(t_{*}, y_{*}\right)}\left[u\left(Z_{\sigma_{A}}\right): \sigma_{U_{1}}<\tau_{Q_{*}}, \sigma_{A}<\tau_{\left[t_{1}-\delta \phi(r), t_{*}\right] \times B\left(x_{1}, 2 r\right)}\right] \\
& \geq \mathbb{P}^{\left(t_{*}, y_{*}\right)}\left(\sigma_{U_{1}}<\tau_{Q_{*}}\right) \inf _{z \in B\left(x_{1}, r / 2\right)} \mathbb{E}^{\left(t_{1}, z\right)}\left[u\left(Z_{\sigma_{A}}\right): \sigma_{A}<\tau_{\left[t_{1}-\delta \phi(r), t_{*}\right] \times B(z, r)}\right] \\
& \geq C_{3} \frac{V\left(x_{1}, \delta_{0}^{2} r\right)}{V\left(x_{1}, C_{0} R\right)} \cdot \xi K C_{1} \inf _{z \in B\left(x_{1}, r / 2\right)} \frac{m \otimes \mu(A)}{V(z, r) \phi(r)} \\
& \geq \frac{C_{1} C_{3} \xi K}{c_{4} \widetilde{C}_{\mu}}\left(\frac{\delta_{0}^{2}}{2}\right)^{d_{2}} \frac{m \otimes \mu(A)}{V\left(x_{1}, C_{0} R\right) \phi(R)},
\end{aligned}
$$

where in the third inequality we used the fact that $\tau_{\left[t_{1}-\delta \phi(r), t_{*}\right] \times B\left(x_{1}, 2 r\right)} \leq \tau_{Q_{*}}$. Since $A$ is an arbitrary compact subset of $\widetilde{A}$, we have by (4.9) that

$$
\frac{m \otimes \mu(\widetilde{A})}{V\left(x_{1}, C_{0} R\right) \phi(R)} \leq \frac{c_{4} \widetilde{C}_{\mu}}{C_{1} C_{3} \xi K}\left(\frac{2}{\delta_{0}^{2}}\right)^{d_{2}}
$$

Thus by (4.7),

$$
\frac{m \otimes \mu(\widetilde{A})}{m \otimes \mu\left(Q^{\downarrow}\left(t_{1}, \delta, x_{1}, r\right)\right)} \leq \frac{c_{4}^{2} \widetilde{C}_{\mu}^{2} C_{0}^{d_{2}}}{\delta C_{1} C_{3} \xi \sqrt{K}}\left(\frac{2}{\delta_{0}^{2}}\right)^{d_{2}}
$$

which is no larger than $\frac{c_{0}^{d_{2}}}{4 \widetilde{C}_{\mu}}$ by taking $K$ sufficiently large. Let

$$
D=\left[t_{1}-\delta \phi(r), t_{1}-\frac{1}{2} \delta \phi(r)\right] \times B\left(x_{1}, c_{0} r\right) \backslash \tilde{A}
$$

and $M=\sup _{(s, y) \in Q^{\downarrow}\left(t_{1}, \delta, x_{1}, 3 r / 2\right)} u(s, y)$. Note that

$$
\frac{m \otimes \mu(\widetilde{D})}{m \otimes \mu\left(Q^{\downarrow}\left(t_{1}, \delta, x_{1}, r\right)\right)}=\frac{\delta \phi(r) V\left(x_{1}, c_{0} r\right)}{2 \delta \phi(r) V\left(x_{1}, r\right)}-\frac{m \otimes \mu(\widetilde{A})}{m \otimes \mu\left(Q^{\downarrow}\left(t_{1}, \delta, x_{1}, r\right)\right)} \geq \frac{c_{0}^{d_{2}}}{4 \widetilde{C}_{\mu}}
$$

We have by (4.8),

$$
\begin{aligned}
K \leq & u\left(t_{1}, x_{1}\right)=\mathbb{E}^{\left(t_{1}, x_{1}\right)}\left[u\left(Z_{\sigma_{D} \wedge \tau_{r}}\right)\right] \\
= & \mathbb{E}^{\left(t_{1}, x_{1}\right)}\left[u\left(Z_{\sigma_{D} \wedge \tau_{r}}\right): \sigma_{D}<\tau_{r}\right]+\mathbb{E}^{\left(t_{1}, x_{1}\right)}\left[u\left(Z_{\sigma_{D} \wedge \tau_{r}}\right): \sigma_{D} \geq \tau_{r}, X_{\tau_{r}} \notin B\left(x_{1}, 3 r / 2\right)\right] \\
& +\mathbb{E}^{\left(t_{1}, x_{1}\right)}\left[u\left(Z_{\sigma_{D} \wedge \tau_{r}}\right): \sigma_{D} \geq \tau_{r}, X_{\tau_{r}} \in B\left(x_{1}, 3 r / 2\right)\right] \\
\leq & \xi K \mathbb{P}^{\left(t_{1}, x_{1}\right)}\left(\sigma_{D}<\tau_{r}\right)+\eta K+M \mathbb{P}^{\left(t_{1}, x_{1}\right)}\left(\sigma_{D} \geq \tau_{r}\right) .
\end{aligned}
$$

Therefore,

$$
M / K \geq \frac{1-\eta-\xi \mathbb{P}^{\left(t_{1}, x_{1}\right)}\left(\sigma_{D}<\tau_{r}\right)}{\mathbb{P}^{\left(t_{1}, x_{1}\right)}\left(\sigma_{D} \geq \tau_{r}\right)} \geq \frac{1-\eta-\xi c_{1}}{1-c_{1}} \geq \frac{1-\left(2 c_{1}\right) / 3}{1-c_{1}}=: 1+2 \beta,
$$

where $\beta=c_{1} /\left(6\left(1-c_{1}\right)\right)$. Consequently, there exists a point $\left(t_{2}, x_{2}\right) \in Q^{\downarrow}\left(t_{1}, \delta, x_{1}, 2 r\right) \subset Q$ such that $u\left(t_{2}, x_{2}\right) \geq(1+\beta) K=: K_{2}$. 
Iterating the procedure above, we can find a sequence of points $\left\{\left(t_{k}, x_{k}\right)\right\}_{k=1}^{\infty}$ in $\left[t_{0}+\right.$ $\left.\delta \phi(C R) / 2, t_{0}+2 \delta \phi(C R)\right) \times B\left(x_{0}, 2 R\right)$ in the following way. Following the above argument with $\left(t_{2}, x_{2}\right)$ and $K_{2}$ in place of $\left(t_{1}, x_{1}\right)$ and $K$ respectively, we obtain that there exists a point $\left(t_{3}, x_{3}\right) \in Q^{\downarrow}\left(t_{2}, \delta, x_{2}, 2 r_{2}\right)$ such that

$$
r_{2}=R K_{2}^{-1 /\left(d_{2}+\beta_{2}\right)}=(1+\beta)^{-1 /\left(d_{2}+\beta_{2}\right)} R K^{-1 /\left(d_{2}+\beta_{2}\right)}
$$

and

$$
u\left(t_{3}, x_{3}\right) \geq(1+\beta) K_{2}=(1+\beta)^{2} K=: K_{3} .
$$

We continue this procedure to obtain a sequence of points $\left\{\left(t_{k}, x_{k}\right)\right\}$ such that $\left(t_{k+1}, x_{k+1}\right) \in$ $Q^{\downarrow}\left(t_{k}, \delta, x_{k}, 2 r_{k}\right)$ with

$$
r_{k}:=R K_{k}^{-1 /\left(d_{2}+\beta_{2}\right)}=(1+\beta)^{-(k-1) /\left(d_{2}+\beta_{2}\right)} R K^{-1 /\left(d_{2}+\beta_{2}\right)},
$$

and

$$
u\left(t_{k+1}, x_{k+1}\right) \geq(1+\beta)^{k} K=: K_{k+1} .
$$

As $0 \leq t_{k}-t_{k+1} \leq \delta \phi\left(2 r_{k}\right)$ and $d\left(x_{k}, x_{k+1}\right) \leq 2 r_{k}$, we can take $K$ large enough (independent of $R$ and $u)$ so that $\left(t_{k}, x_{k}\right) \in\left[t_{0}+\delta \phi(C R) / 2, t_{0}+2 \delta \phi(C R)\right) \times B\left(x_{0}, 2 R\right)$ for all $k$. This is a contradiction because $u\left(t_{k}, x_{k}\right) \geq(1+\beta)^{k-1} K$ goes to infinity as $k \rightarrow \infty$, while $u$ is bounded on $Q$. We conclude that $u$ is bounded by $K$ in $Q_{-}$. The proof is complete.

Remark 4.4. In [BBK2, Proposition 3.3], another proof of parabolic Harnack inequalities is given by using the Balayage formula. We point out that there is a minor error in its proof there. Indeed, in (3.7) and (3.8) of BBK2 and in lines 5 and 6 from the bottom of p. 307 in BBK2, the summations should be taken over $G-B^{\prime}$ instead of $B-B^{\prime}$. With these corrections, the proof of [BBK2, Proposition 3.3] goes through.

Finally, we prove that under $\operatorname{NDL}(\phi), \mathrm{J}_{\phi, \leq}$ is equivalent to $\operatorname{UHK}(\phi)$, which immediately yields that $\mathrm{NDL}(\phi)+\mathrm{UJS} \Longleftrightarrow \mathrm{PHI}^{+}(\phi)$.

Proposition 4.5. Assume that VD, (1.11) and RVD hold. Then,

$$
\mathrm{NDL}(\phi)+\mathrm{J}_{\phi, \leq} \Longleftrightarrow \mathrm{NDL}(\phi)+\mathrm{UHK}(\phi)
$$

and so

$$
\operatorname{NDL}(\phi)+\mathrm{UJS} \Longleftrightarrow \mathrm{PHI}^{+}(\phi) \Longleftrightarrow \mathrm{PHI}(\phi) .
$$

Proof. First, note that the process $\left\{X_{t}\right\}$ is conservative due to NDL $(\phi)$ (see Proposition 2.4). On the one hand, by Theorem 1.12, UHK $(\phi)$ implies $\mathrm{J}_{\phi,<}$. On the other hand, according to Proposition 3.5, under VD, (1.11) and RVD, NDL $(\phi)$ implies FK $(\phi)$ and $\mathrm{E}_{\phi}$. In particular, the process $\left\{X_{t}\right\}$ possesses a heat kernel. Thus we have by [CKW1, Theorem 4.25] that $\operatorname{NDL}(\phi)+\mathrm{J}_{\phi, \leq}$ imply $\operatorname{UHKD}(\phi)$. Furthermore, by Theorem 1.12, NDL $(\phi)+\mathrm{J}_{\phi, \leq}$ imply $\operatorname{UHK}(\phi)$. This proves (4.10).

By Corollary 3.4. NDL $(\phi)+$ UJS $\Longrightarrow \mathrm{J}_{\phi, \leq}$, which along with (4.10) gives us

$$
\mathrm{NDL}(\phi)+\mathrm{UJS} \Longleftrightarrow \mathrm{UHK}(\phi)+\mathrm{NDL}(\phi)+\mathrm{UJS} .
$$

It now follows from Propositions 3.2 and 3.3 , and Theorem 4.3 that

$$
\mathrm{PHI}(\phi) \Longrightarrow \mathrm{NDL}(\phi)+\mathrm{UJS} \Longrightarrow \mathrm{PHI}^{+}(\phi) \text {. }
$$

This establishes assertion (4.11) as $\mathrm{PHI}^{+}(\phi) \Longrightarrow \mathrm{PHI}(\phi)$. 


\section{$4.2 \mathrm{PHI}(\phi) \Longleftrightarrow \operatorname{PHR}(\phi)+\mathrm{E}_{\phi}+\mathrm{UJS} \Longleftrightarrow \mathrm{EHR}+\mathrm{E}_{\phi}+\mathrm{UJS}$}

The main contribution of this subsection is the following relations among $\operatorname{PHI}(\phi), \operatorname{PHR}(\phi)$ and EHR, which establish the equivalences among (1), (5) and (6) of Theorem 1.20

Theorem 4.6. Assume that $\mu$ and $\phi$ satisfy VD, RVD and (1.11) respectively. Then

$$
\operatorname{PHI}(\phi) \Longleftrightarrow \operatorname{PHR}(\phi)+\mathrm{E}_{\phi}+\mathrm{UJS} \Longleftrightarrow \mathrm{EHR}+\mathrm{E}_{\phi}+\mathrm{UJS} .
$$

We start with the following key lemma.

Lemma 4.7. Under VD and (1.11), EHR and $\mathrm{E}_{\phi, \leq}$ imply $\mathrm{FK}(\phi)$.

Proof. According to Remark 1.16(ii), throughout this subsection we may and do assume that the constant $\varepsilon=1 / 2$ in the definition of EHR.

For any open subset $D$ of $M$, let $G^{D}$ be the associated Green operator. Recall that for any open set $D$, it holds that

$$
\lambda_{1}(D)^{-1} \leq \sup _{x \in D \cap M_{0}} \mathbb{E}^{x} \tau_{D}=\sup _{x \in D \cap M_{0}} G^{D} \mathbf{1}(x) .
$$

For any ball $B=B(x, R) \subset M$ with $x \in M$ and $R>0$, and any open set $D \subset B$, we will verify that

$$
\sup _{x \in D \cap M_{0}} \mathbb{E}^{x} \tau_{D} \leq c \phi(R)\left(\frac{\mu(D)}{V(x, R)}\right)^{\nu},
$$

where $c>0$ and $\nu \in(0,1)$ are two constants independent of $D$ and $B$. Once this is proved, $\mathrm{FK}(\phi)$ immediately follows from (4.12) and (4.13).

Fix an arbitrary $x_{0} \in D \cap M_{0}$. Let $R_{k}=2 \delta^{k} R$ for $k \geq 0$, where $\delta \in(0,1 / 2]$ is a constant to be determined later. Set $B_{k}=B\left(x_{0}, R_{k}\right)$ for $k \geq 0$. Clearly $D \subset B_{0}=B\left(x_{0}, 2 R\right)$. Since $\left(G^{B_{k}}-G^{B_{k+1}}\right) \mathbf{1}_{D}$ is a bounded non-negative function that is harmonic in $B_{k+1}$, we have by EHR and the $\mu$-symmetry of the Green operator $G^{B_{k}}$ that for any positive integers $n>k \geq 0$,

$$
\begin{aligned}
& \sup _{y \in B_{n+1} \cap M_{0}}\left(G^{B_{k}}-G^{B_{k+1}}\right) \mathbf{1}_{D}(y) \\
& \leq \inf _{y \in B_{n+1} \cap M_{0}}\left(G^{B_{k}}-G^{B_{k+1}}\right) \mathbf{1}_{D}(y)+c_{1} \delta^{(n-k) \theta} \sup _{y \in B_{n+1} \cap M_{0}}\left|\left(G^{B_{k}}-G^{B_{k+1}}\right) \mathbf{1}_{D}(y)\right| \\
& \leq \frac{1}{\mu\left(B_{n+1}\right)} \int_{B_{n+1}}\left(G^{B_{k}}-G^{B_{k+1}}\right) \mathbf{1}_{D}(y) \mu(d y)+c_{1} \delta^{(n-k) \theta} \sup _{y \in B_{k} \cap M_{0}}\left|G^{B_{k}} \mathbf{1}_{D}(y)\right| \\
& \leq \frac{1}{\mu\left(B_{n+1}\right)} \int \mathbf{1}_{B_{k} \cap D}(y) G^{B_{k}} \mathbf{1}_{B_{n+1}}(y) \mu(d y)+c_{1} \delta^{(n-k) \theta} \sup _{y \in B_{k} \cap M_{0}}\left|G^{B_{k}} \mathbf{1}(y)\right| \\
& \leq \frac{1}{V\left(x_{0}, R_{n+1}\right)} \mu(D)\left\|G^{B_{k}} \mathbf{1}\right\|_{\infty}+c_{1} \delta^{(n-k) \theta} \sup _{y \in B_{k} \cap M_{0}}\left|G^{B_{k}} \mathbf{1}(y)\right|,
\end{aligned}
$$

where $c_{1}=2^{\theta} c>0$, and $c$ and $\theta \in(0,1]$ are the constants in EHR. On the other hand, we have by $\mathrm{E}_{\phi, \leq}$ that

$$
\sup _{y \in B_{k} \cap M_{0}}\left|G^{B_{k}} \mathbf{1}(y)\right| \leq \sup _{y \in B_{k} \cap M_{0}} \mathbb{E}^{y} \tau_{B\left(y, 2 R_{k}\right)} \leq c_{2} \phi\left(2 R_{k}\right) .
$$


Taking $k=0$ and $n=1$ in (4.14) and $k=1$ in (4.15), we find by (1.9) from VD and (1.11) that

$$
\begin{aligned}
\mathbb{E}^{x_{0}} \tau_{D} & \leq \sup _{y \in B_{2} \cap M_{0}} G^{B_{0}} \mathbf{1}_{D}(y) \\
& \leq \sup _{y \in B_{2} \cap M_{0}}\left(G^{B_{0}}-G^{B_{1}}\right) \mathbf{1}_{D}(y)+\sup _{y \in B_{2} \cap M_{0}} G^{B_{1}} \mathbf{1}(y) \\
& \leq c_{3}\left(\frac{\mu(D)}{V\left(x_{0}, R_{2}\right)}+\delta^{\theta}\right) \phi\left(2 R_{0}\right)+c_{2} \phi\left(2 R_{1}\right) \\
& \leq c_{4}\left(\frac{\mu(D)}{V\left(x_{0}, 2 R\right)} \delta^{-2 d_{2}}+\delta^{\theta}\right) \phi(R)+c_{4} \phi(R) \delta^{\beta_{1}} \\
& \leq c_{5} \phi(R)\left(\frac{\mu(D)}{V(x, R)} \delta^{-2 d_{2}}+\delta^{\theta \wedge \beta_{1}}\right) .
\end{aligned}
$$

Define $\nu=\frac{\theta \wedge \beta_{1}}{2 d_{2}+\theta \wedge \beta_{1}}$. If $\frac{\mu(D)}{V(x, R)} \leq(1 / 2)^{2 d_{2}+\theta \wedge \beta_{1}}$, we take $\delta=\left(\frac{\mu(D)}{V(x, R)}\right)^{1 /\left(2 d_{2}+\theta \wedge \beta_{1}\right)}$, which is no larger than $1 / 2$, in (4.16) to deduce

$$
\mathbb{E}^{x_{0}} \tau_{D} \leq 2 c_{5} \phi(R)\left(\frac{\mu(D)}{V(x, R)}\right)^{\nu}
$$

If $\frac{\mu(D)}{V(x, R)}>(1 / 2)^{2 d_{2}+\theta \wedge \beta_{1}}$, we get from $\mathrm{E}_{\phi, \leq}$ that

$$
\mathbb{E}^{x_{0}} \tau_{D} \leq c_{6} \phi(R)\left(\frac{\mu(D)}{V(x, R)}\right)^{\nu}
$$

Since $x_{0} \in D \cap M_{0}$ is arbitrary, this establishes (4.13) and hence completes the proof.

By VD, (1.11) and [CKW1, Proposition 7.3], FK $(\phi)$ implies the existence of the Dirichlet heat kernel $p^{D}(t, \cdot, \cdot)$ for any bounded open subset $D \subset M$, and that there is a constant $C_{\nu}>0$ such that for every $x_{0} \in D$ and $t>0$

$$
\operatorname{ess}_{\sup } \operatorname{su}_{x, y \in D} p^{D}(t, x, y) \leq \frac{C_{\nu}}{V\left(x_{0}, r\right)}\left(\frac{\phi(r)}{t}\right)^{1 / \nu}
$$

where $r=\operatorname{diam}(D)$, the diameter of $D$.

In the following, we will deduce $\operatorname{NDL}(\phi)$ from $\mathrm{EHR}$ and $\mathrm{E}_{\phi, \leq}$, through establishing the space regularity of Dirichlet heat kernel. This basic approach is due to [HS, Lemma 3.8, Lemma 3.9 and Proposition 3.5]. The arguments below is also motivated by these in [GT, Subsections 5.3 and 5.4].

Lemma 4.8. Assume that (1.11), EHR and $\mathrm{E}_{\phi, \leq}$ are satisfied. Let $D$ be a bounded open subset of $M$. Let $t>0, x \in D \backslash \mathcal{N}$ and $0<r_{1}<\phi^{-1}(t)$ such that $0<r_{1} \leq r / 2$ and $B(x, r) \subset D$, where $r=\left(\phi^{-1}(t)^{\beta_{1}} r_{1}^{\theta}\right)^{1 /\left(\beta_{1}+\theta\right)}, \beta_{1}$ is the constant in (1.11) and $\theta$ is the Hölder exponent in EHR. Then,

$$
\operatorname{ess~osc}_{y \in B\left(x, r_{1}\right)} p^{D}(t, x, y) \leq C\left(\frac{r_{1}}{\phi^{-1}(t)}\right)^{\kappa} \operatorname{ess} \sup _{y \in D} p^{D}(t / 2, y, y)
$$

where $\kappa=\beta_{1} \theta /\left(\beta_{1}+\theta\right)$, and $C$ is a constant depending on the constants in (1.11) and $\mathrm{E}_{\phi, \leq}$. 
Proof. The proof uses some ideas from but is more direct than that of [GT, Lemma 5.10]. For fixed $x \in D \backslash \mathcal{N}$ and $s>0$, set $u(s, y)=p^{D}(s, x, y)$. According to Lemma 4.7 and (4.17),

$$
\int_{D} u(s, y)^{2} \mu(d y)=p^{D}(2 s, x, x)<\infty .
$$

Since, by the symmetry of $p^{D}(t / 2, z, x)=p^{D}(t / 2, x, z)$,

$$
u(t, y)=\int_{D} p^{D}(t / 2, y, z) p^{D}(t / 2, z, x) \mu(d z)=P_{t / 2}^{D} u(t / 2, \cdot)(y),
$$

we have $u(t, \cdot) \in \operatorname{Dom}\left(\mathcal{L}^{D}\right) \subset \mathcal{F}^{D}$ for every $t>0$. Thus for $\mu$-a.e. $y \in D$,

$$
\begin{aligned}
\partial_{t} u(t, y) & =\mathcal{L}^{D} P_{t / 2}^{D} u(t / 2, \cdot)(y)=P_{t / 2}^{D} \mathcal{L}^{D} u(t / 2, \cdot)(y) \\
& =\int_{D} p^{D}(t / 2, y, z) \mathcal{L}^{D} u(t / 2, \cdot)(z) \mu(d z)=-\mathcal{E}\left(p^{D}(t / 2, y, \cdot), u(t / 2, \cdot)\right) .
\end{aligned}
$$

Hence, by the Cauchy-Schwarz inequality and the spectral representation,

$$
\begin{aligned}
\left|\partial_{t} u(t, y)\right| & \leq \sqrt{\mathcal{E}\left(p^{D}(t / 2, y, \cdot), p^{D}(t / 2, y, \cdot)\right)} \sqrt{\mathcal{E}(u(t / 2, \cdot), u(t / 2, \cdot))} \\
& =\sqrt{\mathcal{E}\left(P_{t / 4}^{D} p^{D}(t / 4, y, \cdot), P_{t / 4}^{D} p^{D}(t / 4, y, \cdot)\right)} \sqrt{\mathcal{E}\left(P_{t / 4}^{D} u(t / 4, \cdot), P_{t / 4}^{D} u(t / 4, \cdot)\right)} \\
& \leq \sqrt{(2 / t)\left\|p^{D}(t / 4, y, \cdot)\right\|_{L^{2}(D ; \mu)}^{2}} \sqrt{(2 / t)\|u(t / 4, \cdot)\|_{L^{2}(D ; \mu)}^{2}} \\
& =\frac{2}{t} \sqrt{p^{D}(t / 2, y, y) p^{D}(t / 2, x, x)} \leq \frac{2}{t} \operatorname{ess} \sup _{D \backslash \mathcal{N}} p^{D}(t / 2, y, y) .
\end{aligned}
$$

In particular, by (4.17), $f(t, y):=\partial_{t} u(t, y)$ is a bounded function on $D$ for every $t>0$. Note that $\lim _{s \rightarrow \infty} p^{D}(s, x, y)=0$ for every $y \in D \backslash \mathcal{N}$, also thanks to (4.17). Then we have

$$
\begin{aligned}
u(t, y) & =-\int_{t}^{\infty} \partial_{s} p^{D}(s, x, y) d s=-\int_{0}^{\infty} \partial_{t} p^{D}(t+r, x, y) d r \\
& =-\int_{0}^{\infty} \int_{D} p^{D}(r, y, z) \partial_{t} p^{D}(t, x, z) \mu(d z) d r=-G^{D} f(t, \cdot)(y) .
\end{aligned}
$$

Hence, by EHR, Lemma 3.9 and $\mathrm{E}_{\phi, \leq}$, for any $0<r_{1} \leq r / 2$,

$$
\begin{aligned}
\operatorname{ess}_{B\left(x, r_{1}\right)} u(t, \cdot) & \leq 2 \sup _{y \in B(x, r) \backslash \mathcal{N}}|f(t, y)| \sup _{y \in B(x, r) \backslash \mathcal{N}} \mathbb{E}^{y} \tau_{B(x, r)}+c_{1}\left(\frac{r_{1}}{r}\right)^{\theta} \sup _{y \in D \backslash \mathcal{N}}|u(t, y)| \\
& \leq c_{2}\left[\phi(r) \frac{A}{t}+\left(\frac{r_{1}}{r}\right)^{\theta} A\right],
\end{aligned}
$$

where $A=\sup _{z \in D \backslash \mathcal{N}} p^{D}(t / 2, z, z)$. In the last inequality above, we also used the facts that $\sup _{y, z \in D \backslash \mathcal{N}} p^{D}(t, y, z)=\sup _{z \in D \backslash \mathcal{N}} p^{D}(t, z, z)$ and $t \mapsto \sup _{z \in D \backslash \mathcal{N}} p^{D}(t, z, z)$ is a decreasing function, see e.g., the proof of Lemma [CKW1, Lemma 7.9].

For any $0<r<\phi^{-1}(t)$, by (1.11),

$$
\frac{\phi(r)}{t} \leq c_{3}\left(\frac{r}{\phi^{-1}(t)}\right)^{\beta_{1}},
$$


whence it follows that for any $0<r_{1} \leq r / 2$ and $0<r<\phi^{-1}(t)$,

$$
\operatorname{ess}_{B\left(x, r_{1}\right)} u \leq C\left[\left(\frac{r}{\phi^{-1}(t)}\right)^{\beta_{1}}+\left(\frac{r_{1}}{r}\right)^{\theta}\right] A \text {. }
$$

By choosing $r=\left(\phi^{-1}(t)^{\beta_{1}} r_{1}^{\theta}\right)^{1 /\left(\beta_{1}+\theta\right)}$ in the inequality above, we proved the desired assertion.

Lemma 4.9. Suppose that VD, (1.11), EHR and $\mathrm{E}_{\phi, \leq}$ hold. Then for any $x \in M_{0}, t>0$ and $0<r \leq 2^{-\left(\beta_{1}+\theta\right) / \beta_{1}} \phi^{-1}(t)$ the following estimate holds

$$
\left|p^{B\left(x, \phi^{-1}(t)\right)}(t, x, x)-p^{B\left(x, \phi^{-1}(t)\right)}(t, x, y)\right| \leq\left(\frac{r}{\phi^{-1}(t)}\right)^{\kappa} \frac{C}{V\left(x, \phi^{-1}(t)\right)}, \quad y \in B(x, r) \backslash \mathcal{N},
$$

where $\beta_{1}$ is the constant in (1.11), $\theta$ is the Hölder exponent in EHR, and $\kappa$ is the constant in Lemma 4.8.

Proof. Fix $x \in M_{0}$ and $t, r_{1}>0$ with $0<r_{1} \leq 2^{-\left(\beta_{1}+\theta\right) / \beta_{1}} \phi^{-1}(t)$. We choose $r=$ $\left(\phi^{-1}(t)^{\beta_{1}} r_{1}^{\theta}\right)^{1 /\left(\beta_{1}+\theta\right)}$ as in Lemma 4.8. Then, $0<r_{1} \leq r / 2$. By applying Lemma 4.8 with $D=B\left(x, \phi^{-1}(t)\right)$, we get

$$
\operatorname{ess~osc}_{y \in B\left(x, r_{1}\right)} p^{B\left(x, \phi^{-1}(t)\right)}(t, x, y) \leq C\left(\frac{r_{1}}{\phi^{-1}(t)}\right)^{\kappa} \operatorname{ess} \sup _{y \in B\left(x, \phi^{-1}(t)\right)} p^{B\left(x, \phi^{-1}(t)\right)}(t / 2, y, y) .
$$

This along with (4.17) yields the desired assertion.

Having all the lemmas at hand, we can obtain the following result.

Proposition 4.10. Let VD, (1.11), EHR and $\mathrm{E}_{\phi}$ be satisfied. Then for any open subset $D \subset M$, the semigroup $\left\{P_{t}^{D}\right\}$ possesses the heat kernel $p^{D}(t, x, y)$, and moreover $\operatorname{NDL}(\phi)$ holds true.

Proof. The existence of heat kernel $p^{D}(t, x, y)$ associated with the semigroup $\left\{P_{t}^{D}\right\}$ for any open subset $D \subset M$ has been stated in the remark below Lemma 4.7, and so we only need to verify $\operatorname{NDL}(\phi)$.

According to $\mathrm{E}_{\phi}$ and [CKW1, Lemma 4.17], there are constants $\varepsilon \in(0,1)$ and $\delta \in(0,1 / 2)$ such that for all $x \in M_{0}$ and for any $t, r>0$ with $t \leq \delta \phi(r), \mathbb{P}^{x}\left(\tau_{B(x, r)} \leq t\right) \leq \varepsilon$. In the following, let $B=B(x, r)$ and $0<t \leq \delta \phi(r)$. Then for any $x \in B \backslash \mathcal{N}$, since the process $\left\{X_{t}\right\}$ has no killings inside $M$,

$$
\int_{B} p^{B}(t, x, y) \mu(d y)=\mathbb{P}^{x}\left(\tau_{B}>t\right) \geq 1-\varepsilon
$$

Therefore,

$$
p^{B}(2 t, x, x)=\int_{B} p^{B}(t, x, y)^{2} \mu(d y) \geq \frac{1}{\mu(B)}\left(\int_{B} p^{B}(t, x, y) \mu(d y)\right)^{2} \geq \frac{c_{1}}{V(x, r)} .
$$


In particular, taking $r=\phi^{-1}(t / \delta)>0$ in the inequality above, we arrive at

$$
p^{B\left(x, \phi^{-1}(t /(2 \delta))\right)}(t, x, x) \geq \frac{c_{2}}{V\left(x, \phi^{-1}(t)\right)} .
$$

Furthermore, according to Lemma 4.9, VD and (1.11), there exists a constant $c_{3}>0$ such that for any $0<r \leq 2^{-\left(\beta_{1}+\theta\right) / \beta_{1}} \phi^{-1}(t /(2 \delta))$, we have

$\left|p^{B\left(x, \phi^{-1}(t /(2 \delta))\right)}(t, x, x)-p^{B\left(x, \phi^{-1}(t /(2 \delta))\right)}(t, x, y)\right| \leq\left(\frac{r}{\phi^{-1}(t)}\right)^{\kappa} \frac{c_{3}}{V\left(x, \phi^{-1}(t)\right)}, \quad y \in B(x, r) \backslash \mathcal{N}$,

where $\beta_{1}$ is the constant in (1.11), $\theta$ is the Hölder exponent in EHR, and $\kappa$ is the constant in Lemma 4.8.

Combining with both inequalities above and choosing $\eta \in(0,1)$ small enough such that $\eta^{\kappa} c_{3} \leq \frac{1}{2} c_{2}$ and $\eta \phi^{-1}(t) \leq 2^{-\left(\beta_{1}+\theta\right) / \beta_{1}} \phi^{-1}(t /(2 \delta))$ for all $t>0$, one can get that for any $x \in M_{0}$ and $y \in B\left(x, \eta \phi^{-1}(t)\right) \backslash \mathcal{N}$,

$$
\begin{aligned}
& p^{B\left(x, \phi^{-1}(t /(2 \delta))\right)}(t, x, y) \\
& \geq p^{B\left(x, \phi^{-1}(t /(2 \delta))\right)}(t, x, x)-\left|p^{B\left(x, \phi^{-1}(t /(2 \delta))\right)}(t, x, x)-p^{B\left(x, \phi^{-1}(t /(2 \delta))\right)}(t, x, y)\right| \\
& \geq \frac{c_{2}}{2 V\left(x, \phi^{-1}(t)\right)} .
\end{aligned}
$$

That is, thanks to VD and (1.11) again, there are constants $c_{i}>0(i=4,5,6)$ such that $0<2 c_{4} \leq c_{5}$ and for any $x \in M_{0}$ and $y \in B\left(x, 2 c_{4} \phi^{-1}(t)\right) \backslash \mathcal{N}$,

$$
p^{B\left(x, c_{5} \phi^{-1}(t)\right)}(t, x, y) \geq \frac{c_{6}}{V\left(x, \phi^{-1}(t)\right)} .
$$

Now, for any $x_{0} \in M$ and $r, t>0$ such that $\left(c_{4}+c_{5}\right) \phi^{-1}(t) \leq r$, we have $B\left(x, c_{5} \phi^{-1}(t)\right) \subset$ $B\left(x_{0}, r\right)$ for all $x \in B\left(x_{0}, c_{4} \phi^{-1}(t)\right)$, and so

$$
p^{B\left(x_{0}, r\right)}(t, x, y) \geq p^{B\left(x, c_{5} \phi^{-1}(t)\right)}(t, x, y) \geq \frac{c_{6}}{V\left(x, \phi^{-1}(t)\right)}, \quad x, y \in B\left(x_{0}, c_{4} \phi^{-1}(t)\right) \backslash \mathcal{N} .
$$

This proves that $\operatorname{NDL}(\phi)$ holds true with $\varepsilon=c_{4} \wedge \frac{1}{c_{4}+c_{5}}$.

Note that by Proposition 4.10 and Proposition 2.4, EHR $+\mathrm{E}_{\phi}$ imply the conservativeness of the process $X=\left\{X_{t} ; t \geq 0\right\}$ (see Proposition 2.4).

Next, we present the proof of Theorem 4.6.

Proof of Theorem 4.6. That $\operatorname{PHI}(\phi) \Longrightarrow \mathrm{NDL}(\phi)+\mathrm{E}_{\phi}+\mathrm{UJS}+\mathrm{J}_{\phi, \leq}$ has been established in Subsection 3.1, where RVD is used. Since NDL $+\mathrm{E}_{\phi, \leq}+\mathrm{J}_{\phi, \leq} \Longrightarrow \operatorname{PHR}(\phi)$ by Proposition 3.8, we have $\operatorname{PHI}(\phi)$ implies $\operatorname{PHR}(\phi)+\mathrm{E}_{\phi}+$ UJS.

On the other hand, by Proposition 4.10 and (4.11) (where RVD is used too), we have

$$
\mathrm{EHR}+\mathrm{E}_{\phi}+\mathrm{UJS} \Longrightarrow \mathrm{NDL}(\phi)+\mathrm{UJS} \Longleftrightarrow \mathrm{PHI}(\phi)
$$

This completes the proof of the theorem. 


\section{3 $\mathrm{PI}(\phi)+\mathrm{J}_{\phi, \leq}+\mathrm{CSJ}(\phi)+\mathrm{UJS} \Longleftrightarrow \mathrm{PHI}(\phi)$}

In this subsection, we will prove the above mentioned equivalence in Theorem 1.20. Note that, under VD, (1.11) and $\operatorname{RVD}, \operatorname{PHI}(\phi) \Longrightarrow \mathrm{PI}(\phi)+\mathrm{J}_{\phi, \leq}+\mathrm{CSJ}(\phi)+$ UJS has already been proved by combining the results in Subsection 3.1. Propositions 3.5 and Theorem 1.12, So all we need is to prove the following theorem.

Theorem 4.11. Assume that $\mu$ and $\phi$ satisfy VD, RVD and (1.11) respectively. Then

$$
\mathrm{PI}(\phi)+\mathrm{J}_{\phi, \leq}+\mathrm{CSJ}(\phi)+\mathrm{UJS} \Longrightarrow \operatorname{PHI}(\phi) .
$$

First of all, note that $\operatorname{PI}(\phi)+\mathrm{J}_{\phi, \leq}+\mathrm{CSJ}(\phi)$ imply the conservativeness of the process. Indeed, $\mathrm{PI}(\phi)+$ RVD imply $\mathrm{FK}(\phi)$ by Proposition 2.9 , and $\mathrm{FK}(\phi)+\mathrm{J}_{\phi, \leq}+\mathrm{CSJ}(\phi)$ imply $\mathrm{E}_{\phi}$ by Proposition 2.7. Furthermore, $\mathrm{J}_{\phi, \leq}+\mathrm{E}_{\phi}$ imply the conservativeness of the process (see CKW1, Lemma 4.21]).

To prove the theorem, we begin with the following logarithmic lemma, which plays the key role in the proof of Hölder continuity of harmonic functions. The proof below is motivated by that of [CKP1, Lemma 1.3].

Proposition 4.12. Let $B_{r}=B\left(x_{0}, r\right)$ for some $x_{0} \in M$ and $r>0$. Assume that $u \in \mathcal{F}_{B_{R}}^{\text {loc }}$ is a bounded and superharmonic function in a ball $B_{R}$ such that $u \geq 0$ on $B_{R}$. If VD, (1.11), $\operatorname{CSJ}(\phi)$ and $\mathrm{J}_{\phi, \leq}$ hold, then for any $l>0$ and $0<2 r \leq R$,

$$
\int_{B_{r} \times B_{r}}\left[\log \left(\frac{u(x)+l}{u(y)+l}\right)\right]^{2} J(d x, d y) \leq \frac{c_{1} V\left(x_{0}, r\right)}{\phi(r)}\left(1+\frac{\phi(r)}{\phi(R)} \frac{\text { Tail }\left(u_{-} ; x_{0}, R\right)}{l}\right),
$$

where Tail $\left(u_{-} ; x_{0}, R\right)$ is the nonlocal tail of $u_{-}$in $B\left(x_{0}, R\right)$ defined by (2.2), and $c_{1}$ is a constant independent of $u, x_{0}, r, R$ and $l$.

Proof. According to $\operatorname{CSJ}(\phi), \mathrm{J}_{\phi, \leq}$ and [CKW1, Proposition 2.3(5)], we can choose $\varphi \in$ $\mathcal{F}_{B_{3 r / 2}}$ related to $\operatorname{Cap}\left(B_{r}, B_{3 r / 2}\right)$ such that

$$
\mathcal{E}(\varphi, \varphi) \leq 2 \operatorname{Cap}\left(B_{r}, B_{3 r / 2}\right) \leq \frac{c_{1} V\left(x_{0}, r\right)}{\phi(r)}
$$

Since $u$ is a bounded and superharmonic function in a ball $B_{R}$ and $\frac{\varphi^{2}}{u+l} \in \mathcal{F}_{B_{3 r / 2}}$ for any $l>0$, we have by Theorem 2.2 that

$$
\begin{aligned}
0 \leq & \mathcal{E}\left(u, \frac{\varphi^{2}}{u+l}\right) \\
= & \int_{B_{2 r} \times B_{2 r}}(u(x)-u(y))\left(\frac{\varphi^{2}(x)}{u(x)+l}-\frac{\varphi^{2}(y)}{u(y)+l}\right) J(d x, d y) \\
& +2 \int_{B_{2 r} \times B_{2 r}^{c}}(u(x)-u(y)) \frac{\varphi^{2}(x)}{u(x)+l} J(d x, d y) \\
= & \int_{B_{2 r} \times B_{2 r}}((u(x)+l)-(u(y)+l))\left(\frac{\varphi^{2}(x)}{u(x)+l}-\frac{\varphi^{2}(y)}{u(y)+l}\right) J(d x, d y)
\end{aligned}
$$




$$
\begin{aligned}
& +2 \int_{B_{2 r} \times B_{2 r}^{c}}(u(x)-u(y)) \frac{\varphi^{2}(x)}{u(x)+l} J(d x, d y) \\
= & \int_{B_{2 r} \times B_{2 r}} \varphi(x) \varphi(y)\left(\frac{\varphi(y)}{\varphi(x)}+\frac{\varphi(x)}{\varphi(y)}-\frac{\varphi(x)(u(y)+l)}{\varphi(y)(u(x)+l)}-\frac{\varphi(y)(u(x)+l)}{\varphi(x)(u(y)+l)}\right) J(d x, d y) \\
& +2 \int_{B_{2 r} \times B_{2 r}^{c}}(u(x)-u(y)) \frac{\varphi^{2}(x)}{u(x)+l} J(d x, d y) \\
= & : I_{1}+I_{2} .
\end{aligned}
$$

Applying the inequality

$$
\frac{a}{b}+\frac{b}{a}-2=(a-b)\left(b^{-1}-a^{-1}\right) \geq(\log a-\log b)^{2}, \quad a, b>0
$$

with $a=\frac{u(y)+l}{\varphi(y)}$ and $b=\frac{u(x)+l}{\varphi(x)}$, we find that

$$
\begin{aligned}
& \frac{\varphi(x)(u(y)+l)}{\varphi(y)(u(x)+l)}+\frac{\varphi(y)(u(x)+l)}{\varphi(x)(u(y)+l)}-\frac{\varphi(y)}{\varphi(x)}-\frac{\varphi(x)}{\varphi(y)} \\
& \geq\left(\log \frac{u(y)+l}{\varphi(y)}-\log \frac{u(x)+l}{\varphi(x)}\right)^{2}-\left(\frac{\varphi(y)}{\varphi(x)}+\frac{\varphi(x)}{\varphi(y)}-2\right)
\end{aligned}
$$

and so

$$
\begin{aligned}
I_{1} \leq & -\int_{B_{2 r} \times B_{2 r}} \varphi(x) \varphi(y)\left(\log \frac{u(y)+l}{\varphi(y)}-\log \frac{u(x)+l}{\varphi(x)}\right)^{2} J(d x, d y) \\
& +\int_{B_{2 r} \times B_{2 r}}(\varphi(x)-\varphi(y))^{2} J(d x, d y) .
\end{aligned}
$$

On the other hand, due to the fact that $u \geq 0$ on $B_{R}$, for all $x \in B_{2 r}$ and $y \in B_{R} \backslash B_{2 r}$,

$$
\frac{u(x)-u(y)}{u(x)+l} \leq 1
$$

while for all $x \in B_{2 r}$ and $y \in B_{R}^{c}$,

$$
\frac{u(x)-u(y)}{u(x)+l} \leq \frac{(u(x)-u(y))_{+}}{u(x)+l} \leq \frac{u(x)+u_{-}(y)}{u(x)+l} \leq 1+l^{-1} u_{-}(y) .
$$

Therefore,

$$
I_{2} \leq 2 \int_{B_{2 r} \times B_{2 r}^{c}} \varphi^{2}(x) J(d x, d y)+2 l^{-1} \int_{B_{2 r} \times B_{R}^{c}} u_{-}(y) \varphi^{2}(x) J(d x, d y) .
$$

Combining all the estimates above and the fact that $\varphi=1$ on $B_{r}$, we obtain

$$
\int_{B_{r} \times B_{r}}\left[\log \left(\frac{u(x)+l}{u(y)+l}\right)\right]^{2} J(d x, d y)
$$




$$
\begin{aligned}
\leq & \int_{B_{2 r} \times B_{2 r}} \varphi(x) \varphi(y)\left(\log \frac{u(y)+l}{\varphi(y)}-\log \frac{u(x)+l}{\varphi(x)}\right)^{2} J(d x, d y) \\
\leq & \int_{B_{2 r} \times B_{2 r}}(\varphi(x)-\varphi(y))^{2} J(d x, d y)+2 \int_{B_{2 r} \times B_{2 r}^{c}} \varphi^{2}(x) J(d x, d y) \\
& +2 l^{-1} \int_{B_{2 r} \times B_{R}^{c}} u_{-}(y) \varphi^{2}(x) J(d x, d y) \\
\leq & \mathcal{E}(\varphi, \varphi)+\frac{c_{2} V\left(x_{0}, r\right)}{\phi(R) l} \text { Tail }\left(u_{-} ; x_{0}, R\right),
\end{aligned}
$$

where the last inequality follows from $\mathrm{J}_{\phi, \leq}$ and the fact that for any $x \in B_{3 r / 2}$ and $y \in B_{R}^{c}$ with $R \geq 2 r$,

$$
\frac{V\left(x_{0}, d\left(x_{0}, y\right)\right) \phi\left(d\left(x_{0}, y\right)\right)}{V(x, d(x, y)) \phi(d(x, y))} \leq c^{\prime}\left(1+\frac{d\left(x_{0}, x\right)}{d(x, y)}\right)^{\beta_{2}+\alpha_{2}} \leq c^{\prime}\left(1+\frac{3 r / 2}{R-3 r / 2}\right)^{\beta_{2}+\alpha_{2}} \leq c^{\prime \prime}
$$

thanks to VD and (1.11). Hence, the desired assertion follows from the inequality and (4.18).

For the diffusion case, Proposition 4.12 was originally due to Moser. In that case, one can use the Leibniz rule, but for the jump case some more care is required. See [KZ, Corollary 7.7] for a related inequality. In the following we give another proof that is more robust.

Proof. (Another proof of Proposition 4.12) For a function $v$ on $M$ and for fixed $x, y \in M$, write

$$
\bar{v}(t)=\bar{v}_{x y}(t):=t v(x)+(1-t) v(y), \quad t \in[0,1] .
$$

Take $\varphi \in \mathcal{F}_{B_{3 r / 2}}$ as in (4.18) in the previous proof. For any $x, y \in M$ and $l>0$, it holds that

$$
\begin{aligned}
(u(x)-u(y))\left[\varphi(x)^{2} /(u(x)+l)-\varphi(y)^{2} /(u(y)+l)\right] \\
=\int_{0}^{1}\left[\frac{d}{d t} \frac{\bar{\varphi}^{2}}{(\bar{u}+l)}(s)\right] \frac{d}{d t}(\bar{u}(s)+l) d s \\
=\int_{0}^{1} \frac{2 \bar{\varphi}(s) \frac{d}{d t} \bar{\varphi}(s)}{(\bar{u}(s)+l)} \frac{d}{d t}(\bar{u}(s)+l) d s-\int_{0}^{1}\left[\frac{\bar{\varphi}}{(\bar{u}+l)}(s)\right]^{2}\left[\frac{d}{d t}(\bar{u}(s)+l)\right]^{2} d s \\
=\int_{0}^{1} 2\left[\bar{\varphi}(s) \frac{d}{d t} \bar{\varphi}(s)\right]\left[\frac{d}{d t} \log (\bar{u}(s)+l)\right] d s-\int_{0}^{1} \bar{\varphi}(s)^{2}\left[\frac{d}{d t} \log (\bar{u}(s)+l)\right]^{2} d s .
\end{aligned}
$$

Multiplying $J(x, y)$ and integrating over $B_{2 r} \times B_{2 r}$ w.r.t. $\mu \times \mu$ in both sides of the equality 
above, we have

$$
\begin{aligned}
\int_{B_{2 r} \times B_{2 r}} \int_{0}^{1} \bar{\varphi}(s)^{2}\left[\frac{d}{d t} \log (\bar{u}(s)+l)\right]^{2} d s J(d x, d y) \\
\quad+\mathcal{E}\left(u, \varphi^{2} /(u+l)\right)-2 \int_{B_{2 r} \times B_{2 r}^{c}}(u(x)-u(y)) \frac{\varphi^{2}(x)}{u(x)+l} J(d x, d y) \\
=2 \int_{B_{2 r} \times B_{2 r}} \int_{0}^{1}\left[\bar{\varphi}(s) \frac{d}{d t} \bar{\varphi}(s)\right]\left[\frac{d}{d t} \log (\bar{u}(s)+l)\right] d s J(d x, d y) \\
\leq 2\left[\int_{B_{2 r} \times B_{2 r}} \int_{0}^{1} \bar{\varphi}(s)^{2}\left(\frac{d}{d t} \log (\bar{u}(s)+l)\right)^{2} d s J(d x, d y)\right]^{1 / 2} \\
\times\left[\int_{B_{2 r} \times B_{2 r}} \int_{0}^{1}\left(\frac{d}{d t} \bar{\varphi}(s)\right)^{2} d s J(d x, d y)\right]^{1 / 2} \\
\leq 2\left[\int_{B_{2 r} \times B_{2 r}} \int_{0}^{1} \bar{\varphi}(s)^{2}\left(\frac{d}{d t} \log (\bar{u}(s)+l)\right)^{2} d s J(d x, d y)\right]^{1 / 2} \mathcal{E}(\varphi, \varphi)^{1 / 2} .
\end{aligned}
$$

In the following, we set

$$
K:=\int_{B_{2 r} \times B_{2 r}} \int_{0}^{1} \bar{\varphi}(s)^{2}\left(\frac{d}{d t} \log (\bar{u}(s)+l)\right)^{2} d s J(d x, d y) .
$$

Now, as in the previous proof,

$$
2\left|\int_{B_{2 r} \times B_{2 r}^{c}}(u(x)-u(y)) \frac{\varphi^{2}(x)}{u(x)+l} J(d x, d y)\right|=\left|I_{2}\right| \leq \mathcal{E}(\varphi, \varphi)+\frac{c_{2} V\left(x_{0}, r\right)}{\phi(R) l} \operatorname{Tail}\left(u_{-} ; x_{0}, R\right) .
$$

Further, noting that $u+l$ is bounded and superharmonic on $2 B$, we have by Theorem 2.2 that $\mathcal{E}\left(u, \varphi^{2} /(u+l)\right) \geq 0$. Plugging these and (4.18) into (4.19), we have

$$
K-\frac{c_{1} V\left(x_{0}, r\right)}{\phi(r)}-\frac{c_{2} V\left(x_{0}, r\right)}{\phi(R) l} \text { Tail }\left(u_{-} ; x_{0}, R\right) \leq 2 K^{1 / 2}\left(\frac{c_{1} V\left(x_{0}, r\right)}{\phi(r)}\right)^{1 / 2} .
$$

We thus obtain

$$
K \leq \frac{c_{3} V\left(x_{0}, r\right)}{\phi(r)}\left[1+\frac{\phi(r)}{\phi(R)} \frac{\text { Tail }\left(u_{-} ; x_{0}, R\right)}{l}\right] .
$$

On the other hand, since $\varphi=1$ on $B_{r}$, using the Cauchy-Schwarz inequality we have

$$
\begin{aligned}
K & \geq \int_{B_{2 r} \times B_{2 r}}\left(\varphi(x)^{2} \wedge \varphi(y)^{2}\right) \int_{0}^{1}\left[\frac{d}{d t} \log (\bar{u}(s)+l)\right]^{2} d s J(d x, d y) \\
& \geq \int_{B_{2 r} \times B_{2 r}}\left(\varphi(x)^{2} \wedge \varphi(y)^{2}\right)\left[\int_{0}^{1} \frac{d}{d t} \log (\bar{u}(s)+l) d s\right]^{2} J(d x, d y) \\
& \geq \int_{B_{r} \times B_{r}}[\log (u(y)+l)-\log (u(x)+l)]^{2} J(d x, d y) .
\end{aligned}
$$

We therefore prove the desired inequality, by combining all the inequalities above. 
As a consequence of Proposition 4.12, we have the following statement.

Corollary 4.13. Let $B_{r}=B\left(x_{0}, r\right)$ for some $x_{0} \in M$ and $r>0$. Assume that $u \in \mathcal{F}_{B_{R}}^{\text {loc }}$ is a bounded and superharmonic function in a ball $B_{R}$ such that $u \geq 0$ on $B_{R}$. For any $a, l>0$ and $b>1$, define

$$
v=\left[\log \left(\frac{a+l}{u+l}\right)\right]_{+} \wedge \log b .
$$

If $\mathrm{VD}$, (1.11), $\mathrm{CSJ}(\phi), \mathrm{J}_{\phi, \leq}$ and $\mathrm{PI}(\phi)$ hold, then for any $l>0$ and $0<2 \kappa r \leq R$,

$$
\frac{1}{V\left(x_{0}, r\right)} \int_{B_{r}}\left(v-\bar{v}_{B_{r}}\right)^{2} d \mu \leq c_{1}\left(1+\frac{\phi(r)}{\phi(R)} \frac{\text { Tail }\left(u_{-} ; x_{0}, R\right)}{l}\right)
$$

where $\kappa \geq 1$ is the constant in $\operatorname{PI}(\phi), \bar{v}_{B_{r}}=\frac{1}{\mu\left(B_{r}\right)} \int_{B_{r}} v d \mu$ and $c_{1}$ is a constant independent of $u, x_{0}, r, R$ and $l$.

Proof. By $\operatorname{PI}(\phi)$ and (1.11), we have

$$
\int_{B_{r}}\left(v-\bar{v}_{B_{r}}\right)^{2} d \mu \leq c_{2} \phi(r) \int_{B_{\kappa r} \times B_{\kappa r}}(v(x)-v(y))^{2} J(d x, d y) .
$$

Observing that $v$ is a truncation of the sum of a constant and $\log (u+l)$,

$$
\int_{B_{\kappa r} \times B_{\kappa r}}(v(x)-v(y))^{2} J(d x, d y) \leq \int_{B_{\kappa r} \times B_{\kappa r}}\left(\log \left(\frac{u(x)+l}{u(y)+l}\right)\right)^{2} J(d x, d y) .
$$

Hence, it suffices to apply Proposition 4.12 to conclude the assertion.

Proposition 4.14. Let $B_{r}=B\left(x_{0}, r\right)$ for some $x_{0} \in M$ and $r>0$. Assume that $u \in \mathcal{F}_{B_{R}}^{\text {loc }}$ is a bounded and harmonic function in a ball $B_{R}$. If $\mathrm{VD}, \operatorname{RVD},(1.11), \operatorname{CSJ}(\phi), \mathrm{J}_{\phi, \leq}$ and $\operatorname{PI}(\phi)$ hold, there are constants $\gamma \in\left(0, \beta_{1}\right)$ and $c>0$ such that

$$
\operatorname{ess~osc}_{B_{r^{\prime}}} u \leq c\left(\frac{r^{\prime}}{r}\right)^{\gamma}\left[\left(\frac{1}{V\left(x_{0}, 2 r\right)} \int_{B\left(x_{0}, 2 r\right)} u^{2} d \mu\right)^{1 / 2}+\text { Tail }\left(u ; x_{0}, r\right)\right],
$$

where $0<r^{\prime} \leq r<R / 2$. In particular, suppose that VD, RVD and (1.11) hold, then we have

$$
\mathrm{PI}(\phi)+\mathrm{J}_{\phi, \leq}+\operatorname{CSJ}(\phi) \Longrightarrow \mathrm{EHR} .
$$

Proof. (i) First, by $\mathrm{J}_{\phi, \leq}$ and Lemma 2.3, it is easy to see that

$$
\text { Tail }\left(u ; x_{0}, r\right) \leq c^{\prime}\|u\|_{\infty}, \quad r>0 .
$$

Thus, assuming (4.20), there is a constant $c^{\prime \prime}>0$ such that for all $0<r<R / 2$,

$$
\operatorname{ess~osc}_{B_{r}} u \leq c^{\prime \prime}\left(\frac{r}{R}\right)^{\gamma}\|u\|_{\infty} \text {. }
$$


From this, we can easily see that, once (4.20) is proved, EHR is yielded.

(ii) In the following, we mainly prove (4.20). We begin with the argument of CKP1, Theorem 1.2]. Before starting, let us fix some notations. For any $j \geq 0$ and $0<2 r<R$, let $r_{j}=r \sigma^{j}$ and $B_{j}=B_{r_{j}}$, where $\sigma \in(0,1 /(4 \kappa)]$ and $\kappa \geq 1$ is the constant in $\operatorname{PI}(\phi)$. Let us define

$$
w\left(r_{0}\right)=w(r)=2 C_{0}\left[\left(\frac{1}{V\left(x_{0}, 2 r\right)} \int_{B\left(x_{0}, 2 r\right)} u^{2} d \mu\right)^{1 / 2}+\text { Tail }\left(u ; x_{0}, r\right)\right]
$$

with the constant $C_{0}$ given in (2.3) of Proposition 2.6, and

$$
w\left(r_{j}\right)=\left(\frac{r_{j}}{r_{0}}\right)^{\gamma} w\left(r_{0}\right)
$$

for some $\gamma \in\left(0, \beta_{1}\right)$. In order to prove the required assertion, it will suffice to verify that

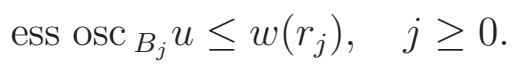

Indeed, for any $0<r^{\prime} \leq r$, we can choose $j \geq 0$ such that $r_{j+1}<r^{\prime} \leq r_{j}$. Then, by (4.21), we have

$$
\operatorname{ess~osc}_{B_{r^{\prime}}} u \leq \operatorname{ess~osc}_{B_{j}} u \leq w\left(r_{j}\right) \leq \sigma^{\gamma}\left(\frac{r_{j+1}}{r}\right)^{\gamma} w(r) \leq \sigma^{\gamma}\left(\frac{r^{\prime}}{r}\right)^{\gamma} w(r)
$$

Thus, the required assertion holds with $c=2 C_{0} \sigma^{\gamma}$.

(iii) We will prove (4.21) by induction. For this, note that $\operatorname{PI}(\phi)+\operatorname{RVD}$ imply $\mathrm{FK}(\phi)$ by Proposition 2.9. Then, according to the definition of $w\left(r_{0}\right)$ and Proposition 2.6, (4.21) holds for $j=0$, since both the functions $u_{+}$and $u_{-}$bounded subharmonic in $B_{R}$.

Now, we make an induction assumption and assume that (4.21) is valid for all $0 \leq i \leq j$ for some $j \geq 0$, and then we prove it holds also for $j+1$. We have that either

$$
\frac{\mu\left(2 B_{j+1} \cap\left\{u \geq \operatorname{ess} \inf _{B_{j}} u+w\left(r_{j}\right) / 2\right\}\right)}{\mu\left(2 B_{j+1}\right)} \geq \frac{1}{2},
$$

or

$$
\frac{\mu\left(2 B_{j+1} \cap\left\{u \leq \operatorname{essinf}_{B_{j}} u+w\left(r_{j}\right) / 2\right\}\right)}{\mu\left(2 B_{j+1}\right)} \geq \frac{1}{2}
$$

must hold. If (4.22) holds, we set $u_{j}:=u-\operatorname{ess~inf}_{B_{j}} u$, and if (4.23) holds, we set $u_{j}:=$ $w\left(r_{j}\right)-\left(u-\operatorname{ess~inf}_{B_{j}} u\right)$. In both cases we have $u_{j} \geq 0$ on $B_{j}$ and

$$
\frac{\mu\left(2 B_{j+1} \cap\left\{u_{j} \geq w\left(r_{j}\right) / 2\right\}\right)}{\mu\left(2 B_{j+1}\right)} \geq \frac{1}{2}
$$

holds. Clearly, $u_{j}$ is bounded and harmonic in $B_{R}$ satisfying that

$$
\begin{aligned}
& \operatorname{ess} \sup _{B_{i}}\left|u_{j}\right| \leq w\left(r_{j}\right)+\operatorname{ess} \sup _{B_{i}}\left|u-\operatorname{ess~inf~}_{B_{j}} u\right| \\
& \leq w\left(r_{i}\right)+\operatorname{ess}_{\sup _{B_{i}}}\left|u-\operatorname{ess~inf}_{B_{i}} u\right|+\left|\operatorname{ess} \inf _{B_{i}} u-\operatorname{ess} \inf _{B_{j}} u\right|
\end{aligned}
$$

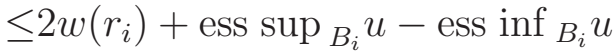

$$
\begin{aligned}
& \leq 3 w\left(r_{i}\right), \quad 0 \leq i \leq j \text {. }
\end{aligned}
$$


We now claim that under the induction assumption we have

$$
\text { Tail }\left(u_{j} ; x_{0}, r_{j}\right) \leq c_{0} \sigma^{-\gamma} w\left(r_{j}\right)
$$

where $c_{0}>0$ is independent of $u, x_{0}, r$ and $\sigma$. Indeed, we have

$$
\begin{aligned}
& \operatorname{Tail}\left(u_{j} ; x_{0}, r_{j}\right)=\phi\left(r_{j}\right) \sum_{i=1}^{j} \int_{B_{i-1} \backslash B_{i}} \frac{\left|u_{j}(x)\right|}{V\left(x_{0}, d\left(x_{0}, x\right)\right) \phi\left(d\left(x_{0}, x\right)\right)} \mu(d x) \\
& +\phi\left(r_{j}\right) \int_{B_{0}^{c}} \frac{\left|u_{j}(x)\right|}{V\left(x_{0}, d\left(x_{0}, x\right)\right) \phi\left(d\left(x_{0}, x\right)\right)} \mu(d x)
\end{aligned}
$$

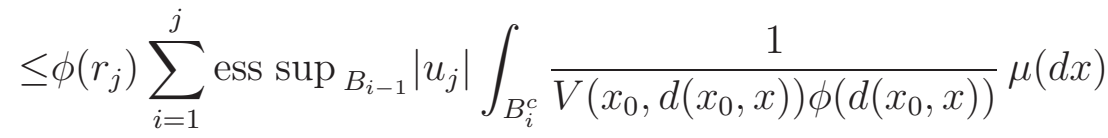

$$
\begin{aligned}
& +\phi\left(r_{j}\right) \int_{B_{0}^{c}} \frac{\left|u_{j}(x)\right|}{V\left(x_{0}, d\left(x_{0}, x\right)\right) \phi\left(d\left(x_{0}, x\right)\right)} \mu(d x) \\
& \leq c_{1} \sum_{i=1}^{j} \frac{\phi\left(r_{j}\right)}{\phi\left(r_{i}\right)} w\left(r_{i-1}\right) \text {, }
\end{aligned}
$$

where in the last inequality we have used (4.25), Lemma 2.3,

$$
\left|u_{j}\right| \leq w\left(r_{0}\right)+\operatorname{ess} \sup _{B_{0}}|u|+|u|, \quad j \geq 0
$$

and

$$
\begin{aligned}
& \int_{B_{0}^{c}} \frac{\left|u_{j}(x)\right|}{V\left(x_{0}, d\left(x_{0}, x\right)\right) \phi\left(d\left(x_{0}, x\right)\right)} \mu(d x) \\
& \leq c^{\prime}\left[\frac{1}{\phi\left(r_{0}\right)}\left(\operatorname{ess} \sup _{B_{0}}|u|+w\left(r_{0}\right)\right)+\int_{B_{0}^{c}} \frac{|u(x)|}{V\left(x_{0}, d\left(x_{0}, x\right)\right) \phi\left(d\left(x_{0}, x\right)\right)} \mu(d x)\right] \\
& \leq c^{\prime \prime} \frac{w\left(r_{0}\right)}{\phi\left(r_{0}\right)} \leq c^{\prime \prime} \frac{w\left(r_{0}\right)}{\phi\left(r_{1}\right)} \text {. }
\end{aligned}
$$

Note that, in the second inequality above we used the fact that

$$
\operatorname{ess} \sup _{B_{0}}|u| \leq \operatorname{ess} \sup _{B_{0}} u^{+}+\operatorname{ess} \sup _{B_{0}} u^{-} \leq w\left(r_{0}\right)
$$

deduced from Proposition 2.6, Estimating further, we have

$$
\begin{aligned}
\sum_{i=1}^{j} \frac{\phi\left(r_{j}\right)}{\phi\left(r_{i}\right)} w\left(r_{i-1}\right) & =w\left(r_{0}\right)\left(\frac{r_{j}}{r_{0}}\right)^{\gamma} \sum_{i=1}^{j} \frac{\phi\left(r_{j}\right)}{\phi\left(r_{i}\right)}\left(\frac{r_{i-1}}{r_{j}}\right)^{\gamma} \\
& \leq c_{2} w\left(r_{0}\right)\left(\frac{r_{j}}{r_{0}}\right)^{\gamma} \sum_{i=1}^{j}\left(\frac{r_{j}}{r_{i}}\right)^{\beta_{1}}\left(\frac{r_{i-1}}{r_{j}}\right)^{\gamma} \\
& =c_{2} w\left(r_{0}\right)\left(\frac{r_{j}}{r_{0}}\right)^{\gamma} \sum_{i=1}^{j}\left(\frac{r_{i-1}}{r_{i}}\right)^{\gamma}\left(\frac{r_{j}}{r_{i}}\right)^{\beta_{1}-\gamma}
\end{aligned}
$$




$$
\leq \frac{c_{2} \sigma^{-\gamma}}{1-\sigma^{\beta_{1}-\gamma}} w\left(r_{j}\right) \leq c_{3} \sigma^{-\gamma} w\left(r_{j}\right)
$$

where we used (1.11) in the first inequality, and used $\sigma \in(0,1 /(4 \kappa)]$ and $\beta_{1}>\gamma$ in the second inequality. Hence, (4.26) is proved with $c_{0}$ independent of $\sigma$.

Next, consider the function $v$ defined as follows

$$
v:=\left[\log \left(\frac{w\left(r_{j}\right) / 2+l}{u_{j}+l}\right)\right]_{+} \wedge k, \quad k, l>0 .
$$

Using the fact $\sigma \in(0,1 /(4 \kappa)]$ again and applying Corollary 4.13 , we get

$$
\frac{1}{\mu\left(2 B_{j+1}\right)} \int_{2 B_{j+1}}\left(v-\bar{v}_{2 B_{j+1}}\right)^{2} d \mu \leq c_{4}\left(1+l^{-1} \frac{\phi\left(r_{j+1}\right)}{\phi\left(r_{j}\right)} \operatorname{Tail}\left(u_{j} ; x_{0}, r_{j}\right)\right) \text {. }
$$

This, along with (4.26) and (1.11), yields that

$$
\frac{1}{\mu\left(2 B_{j+1}\right)} \int_{2 B_{j+1}}\left(v-\bar{v}_{2 B_{j+1}}\right)^{2} d \mu \leq c_{5}\left(1+l^{-1} \sigma^{\beta_{1}-\gamma} w\left(r_{j}\right)\right) .
$$

Hence, choosing $l=\varepsilon w\left(r_{j}\right)$ with $\varepsilon=\sigma^{\beta_{1}-\gamma}$, we get that

$$
\frac{1}{\mu\left(2 B_{j+1}\right)} \int_{2 B_{j+1}}\left(v-\bar{v}_{2 B_{j+1}}\right)^{2} d \mu \leq c_{6} .
$$

To continue, denote in short $\tilde{B}=2 B_{j+1}$. We obtain from (4.24) that

$$
\begin{aligned}
k & =\frac{1}{\mu\left(\tilde{B} \cap\left\{u_{j} \geq w\left(r_{j}\right) / 2\right\}\right)} \int_{\tilde{B} \cap\left\{u_{j} \geq w\left(r_{j}\right) / 2\right\}} k d \mu \\
& =\frac{1}{\mu\left(\tilde{B} \cap\left\{u_{j} \geq w\left(r_{j}\right) / 2\right\}\right)} \int_{\tilde{B} \cap\{v=0\}} k d \mu \\
& \leq \frac{2}{\mu(\tilde{B})} \int_{\tilde{B}}(k-v) d \mu=2\left(k-\bar{v}_{\tilde{B}}\right) .
\end{aligned}
$$

By integrating the preceding inequality over the set $\tilde{B} \cap\{v=k\}$, we further obtain

$$
\frac{\mu(\tilde{B} \cap\{v=k\})}{\mu(\tilde{B})} k \leq \frac{2}{\mu(\tilde{B})} \int_{\tilde{B} \cap\{v=k\}}\left(k-\bar{v}_{\tilde{B}}\right) d \mu \leq \frac{2}{\mu(\tilde{B})} \int_{\tilde{B}}\left|v-\bar{v}_{\tilde{B}}\right| d \mu \leq c_{7},
$$

where (4.27) and the Cauchy-Schwarz inequality are used in the last inequality. Let us take

$$
k=\log \left(\frac{w\left(r_{j}\right) / 2+\varepsilon w\left(r_{j}\right)}{3 \varepsilon w\left(r_{j}\right)}\right)=\log \left(\frac{\frac{1}{2}+\varepsilon}{3 \varepsilon}\right) \approx \log \left(\frac{1}{\varepsilon}\right),
$$

and so we have

$$
\frac{\mu\left(\tilde{B} \cap\left\{u_{j} \leq 2 \varepsilon w\left(r_{j}\right)\right\}\right)}{\mu(\tilde{B})} \leq \frac{c_{7}}{k} \leq \frac{c_{8}}{-\log \sigma}
$$


(iv) We are now in a position to start a suitable iteration to deduce the desired oscillation reduction. From here we make essential changes of the argument in the proof of CKP1, Theorem 1.2]. Note that, in the setting of [CKP1 the proof is heavily based on the fractional Poincaré inequalities (see [CKP1, (5.11)]), which however are not available in the present situation. To deal with this difficulty, we apply Lemma 2.5 instead. In the following, we fix $j \geq 0$. First, for any $i \geq 0$, we define

$$
\varrho_{i}=\left(1+2^{-i}\right) r_{j+1}, \quad B^{i}=B_{\varrho_{i}}
$$

and set

$$
k_{i}=\left(1+2^{-i}\right) \varepsilon w\left(r_{j}\right), \quad w_{i}=\left(k_{i}-u_{j}\right)_{+}, \quad A_{i}=\frac{\mu\left(B^{i} \cap\left\{u_{j} \leq k_{i}\right\}\right)}{\mu\left(B^{i}\right)} .
$$

Then, we have by VD and Lemma 2.5 that

$$
\begin{aligned}
A_{i+2}\left(k_{i+1}-k_{i+2}\right)^{2}= & \frac{1}{\mu\left(B^{i+2}\right)} \int_{B^{i+2} \cap\left\{u_{j} \leq k_{i+2}\right\}}\left(k_{i+1}-k_{i+2}\right)^{2} d \mu \\
\leq & \frac{1}{\mu\left(B^{i+2}\right)} \int_{B^{i+2}} w_{i+1}^{2} d \mu \\
\leq & \frac{c_{8}}{\left(k_{i}-k_{i+1}\right)^{2 \nu}}\left(\frac{1}{\mu\left(B^{i+1}\right)} \int_{B^{i+1}} w_{i}^{2} d \mu\right)^{1+\nu}\left(\frac{\varrho_{i+2}}{\varrho_{i+1}-\varrho_{i+2}}\right)^{\beta_{2}} \\
& \times\left[1+\frac{1}{k_{i}-k_{i+1}}\left(\frac{\varrho_{i+2}}{\varrho_{i+1}-\varrho_{i+2}}\right)^{d_{2}+\beta_{2}-\beta_{1}} \operatorname{Tail}\left(w_{i} ; x_{0}, \varrho_{i+1}\right)\right] \\
\leq & \frac{c_{9}}{\left[\left(2^{-i}-2^{-i-1}\right) \varepsilon w\left(r_{j}\right)\right]^{2 \nu}}\left[\left(\varepsilon w\left(r_{j}\right)\right)^{2} A_{i}\right]^{1+\nu}\left(\frac{1}{2^{-i}-2^{-i-1}}\right)^{d_{2}} \\
& \times\left[1+\frac{1}{\left(2^{-i}-2^{-i-1}\right) \varepsilon w\left(r_{j}\right)}\left(\frac{1}{2^{-i}-2^{-i-1}}\right)^{\left.\operatorname{Tail}\left(w_{i} ; x_{0}, r_{j+1}\right)\right]}\right. \\
\leq & c_{10}\left[\varepsilon w\left(r_{j}\right)\right]^{2} A_{i}^{1+\nu} 2^{\left(1+2 \nu+d_{2}+2 \beta_{2}-\beta_{1}\right) i}\left(1+\frac{1}{\varepsilon w\left(r_{j}\right)} \operatorname{Tail}\left(w_{i} ; x_{0}, r_{j+1}\right)\right),
\end{aligned}
$$

where $\nu$ is the constant in $\operatorname{FK}(\phi)$, and in the third inequality we have used the facts that $u_{j} \geq 0$ on $B^{i+1} \subset B_{j}$ and

$$
\int_{B^{i+1}} w_{i}^{2} d \mu \leq k_{i}^{2} \mu\left(B^{i+1} \cap\left\{w_{i} \geq 0\right\}\right) \leq c^{\prime}\left(\varepsilon w\left(r_{j}\right)\right)^{2} \mu\left(B^{i} \cap\left\{u_{j} \leq k_{i}\right\}\right) .
$$

Hence,

$$
A_{i+2} \leq c_{11} A_{i}^{1+\nu} 2^{\left(3+2 \nu+d_{2}+2 \beta_{2}-\beta_{1}\right) i}\left(1+\frac{1}{\varepsilon w\left(r_{j}\right)} \operatorname{Tail}\left(w_{i} ; x_{0}, r_{j+1}\right)\right) .
$$

Note that, by the facts that $u_{j} \geq 0, w_{i} \leq 2 \varepsilon w\left(r_{j}\right)$ on $B_{j}$ and $\left|w_{i}\right| \leq\left|u_{j}\right|+2 \varepsilon w\left(r_{j}\right)$ on $M$,

$$
\begin{aligned}
\operatorname{Tail}\left(w_{i} ; x_{0}, r_{j+1}\right)= & \phi\left(r_{j+1}\right) \int_{B_{j} \backslash B_{j+1}} \frac{\left|w_{i}(x)\right|}{V\left(x_{0}, d\left(x_{0}, x\right)\right) \phi\left(d\left(x_{0}, x\right)\right)} \mu(d x) \\
& +\frac{\phi\left(r_{j+1}\right)}{\phi\left(r_{j}\right)} \operatorname{Tail}\left(w_{i} ; x_{0}, r_{j}\right)
\end{aligned}
$$




$$
\begin{aligned}
\leq c_{12}\left(2 \varepsilon w\left(r_{j}\right) \phi\left(r_{j+1}\right) \int_{B_{j+1}^{c}} \frac{\mu(d x)}{V\left(x_{0}, d\left(x_{0}, x\right)\right) \phi\left(d\left(x_{0}, x\right)\right)}\right. \\
\quad+2 \varepsilon w\left(r_{j}\right) \phi\left(r_{j+1}\right) \int_{B_{j}^{c}} \frac{\mu(d x)}{V\left(x_{0}, d\left(x_{0}, x\right)\right) \phi\left(d\left(x_{0}, x\right)\right)} \\
\left.\quad+\frac{\phi\left(r_{j+1}\right)}{\phi\left(r_{j}\right)} \operatorname{Tail}\left(u_{j} ; x_{0}, r_{j}\right)\right) \\
\leq c_{12}\left(\varepsilon w\left(r_{j}\right)+\sigma^{\beta_{1}} \operatorname{Tail}\left(u_{j} ; x_{0}, r_{j}\right)\right) \\
\leq c_{13}\left(1+\frac{\sigma^{\beta_{1}-\gamma}}{\varepsilon}\right) \varepsilon w\left(r_{j}\right) \leq 2 c_{13} \varepsilon w\left(r_{j}\right),
\end{aligned}
$$

where the second and the third inequalities follow from Lemma 2.3 and (4.26), respectively, and the last inequality is due to $\varepsilon=\sigma^{\beta_{1}-\gamma}$. Combining with all the conclusions above, we arrive at

$$
A_{i+2} \leq c_{14} A_{i}^{1+\nu} 2^{\left(3+2 \nu+d_{2}+2 \beta_{2}-\beta_{1}\right) i} .
$$

Let $c^{*}=c_{14}^{-1 / \nu} 2^{-\left(3+2 \nu+d_{2}+2 \beta_{2}-\beta_{1}\right) / \nu^{2}}$ and choose the constant $\sigma \in\left(0, \frac{1}{4} \wedge \exp ^{-\left(\frac{c_{8}}{c^{*}}\right)}\right)$. Then, by (4.28),

$$
A_{0} \leq c^{*}=c_{14}^{-1 / \nu} 2^{-\left(3+2 \nu+d_{2}+2 \beta_{2}-\beta_{1}\right) / \nu^{2}} .
$$

According to Lemma 2.10, we can deduce that $\lim _{i \rightarrow \infty} A_{i}=0$. Therefore, $u_{j} \geq \varepsilon w\left(r_{j}\right)$ on $B_{j+1}$, and then we can find that

$\operatorname{ess~osc}_{B_{j+1}} u=\operatorname{ess} \sup _{B_{j+1}} u_{j}-\operatorname{ess~inf~}_{B_{j+1}} u_{j} \leq(1-\varepsilon) w\left(r_{j}\right)=(1-\varepsilon) \sigma^{-\gamma} w\left(r_{j+1}\right)$,

where the inequality above follows from the fact that ess $\sup _{B_{j+1}} u_{j} \leq w\left(r_{j}\right)$, since under (4.22)

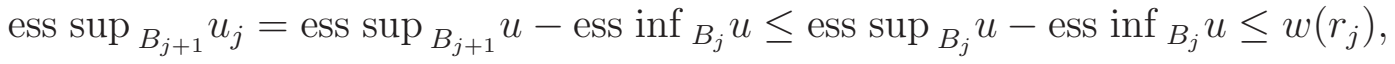

or under (4.23)

$$
\operatorname{ess} \sup _{B_{j+1}} u_{j}=w\left(r_{j}\right)-\operatorname{ess}_{\inf _{B_{j+1}}}\left(u-\operatorname{ess}_{\left.\inf _{B_{j}} u\right) \leq w\left(r_{j}\right) .}\right.
$$

Taking finally $\gamma \in\left(0, \beta_{1}\right)$ small enough such that $\sigma^{\gamma} \geq 1-\varepsilon=1-\sigma^{\beta_{1}-\gamma}$, we obtain that

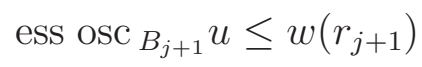

holds, proving the induction step and finishing the proof of (4.21).

We are now in a position to present the proof of the main theorem in this subsection.

Proof of Theorem 4.11. By Theorem 4.6, it suffices to prove that

$$
\mathrm{PI}(\phi)+\mathrm{J}_{\phi, \leq}+\mathrm{CSJ}(\phi)+\mathrm{UJS} \Longrightarrow \mathrm{EHR}+\mathrm{E}_{\phi}+\mathrm{UJS} .
$$

As mentioned in the remark below Theorem 4.11, under VD, RVD and (1.11),

$$
\mathrm{PI}(\phi)+\mathrm{J}_{\phi, \leq}+\mathrm{CSJ}(\phi) \Longrightarrow \mathrm{E}_{\phi}
$$


On the other hand, according to Proposition 4.14 (where RVD is used again),

$$
\operatorname{PI}(\phi)+\mathrm{J}_{\phi, \leq}+\operatorname{CSJ}(\phi) \Longrightarrow \mathrm{EHR} .
$$

The proof is complete.

Remark 4.15. By the proof above and Propositions 4.10 and 3.5, under VD, RVD and (1.11), we have the following relations without using UJS:

$$
\mathrm{PI}(\phi)+\mathrm{J}_{\phi, \leq}+\mathrm{CSJ}(\phi) \Longrightarrow \mathrm{EHR}+\mathrm{E}_{\phi} \Longrightarrow \mathrm{NDL}(\phi) \Longrightarrow \mathrm{PI}(\phi)+\mathrm{E}_{\phi} .
$$

Proof of Corollary 1.21. Assume $\mathrm{PHI}(\phi)$ and $\mathrm{J}_{\phi, \geq}$ are satisfied. Then by Theorem 1.20(4), $\mathrm{J}_{\phi}$ and $\operatorname{CSJ}(\phi)$ hold. So by Theorem 1.11(4), $\operatorname{HK}(\phi)$ also holds.

Conversely, assume $\operatorname{HK}(\phi)$ holds. By Theorem 1.11, $\mathrm{J}_{\phi}$ and $\operatorname{CSJ}(\phi)$ are satisfied. Note that UJS holds trivially because of $\mathrm{J}_{\phi}$. Thus by Theorem 1.20 again, $\mathrm{PHI}(\phi)$ holds.

\section{$5 \quad$ Applications and Examples}

The stability results in Theorem 1.20 allow us to obtain PHI for a large class of symmetric jump processes using "transferring method"; that is, by first establishing PHI for a particular symmetric jump process with jumping kernel $J(x, y)$, we can then use Theorem 1.20 to obtain PHI for other symmetric jump processes whose jumping kernels are comparable to $J(x, y)$. Examples are given in [CKW1, Section 6.1] on fractals that support anomalous diffusions with two-sided heat kernel estimates. The subordination of these diffusion processes enjoy $\operatorname{HK}(\phi)$ and hence $\operatorname{PHI}(\phi)$ by Corollary 1.21, and so can be served as the base examples. For readers' convenience, we give one concrete example here on the Sierpinski gasket.

Example 5.1. (Subordinations of diffusions on fractal-like manifolds.) We first define the 2-dimensional Sierpinski gasket and Brownian motion on it. Let $a_{1}=(0,0), a_{2}=$ $(1,0), a_{3}=(1 / 2, \sqrt{3} / 2)$, and set $F_{i}(x)=\left(x-a_{i}\right) / 2+a_{i}$ for $i=1,2,3$. Then, there exists unique non-void compact set such that $K=\cup_{i=1}^{3} F_{i}(K)$; we call $K$ the 2-dimensional Sierpinski gasket. Let $V_{0}=\left\{a_{1}, a_{2}, a_{3}\right\}$ and set

$$
V_{k}:=\bigcup_{1 \leq i_{1}, \cdots, i_{k} \leq 3} F_{i_{1}} \circ \cdots \circ F_{i_{k}}\left(V_{0}\right), \quad \hat{K}_{\mathrm{pre}}:=\bigcup_{k \geq 0} 2^{k} V_{k} \text { and } \hat{K}:=\bigcup_{k \geq 0} 2^{k} K .
$$

$\hat{K}_{\text {pre }}$ is called a pre-gasket, and $\hat{K}$ is called an unbounded gasket. Let $d(\cdot, \cdot)$ be the geodesic distance on $\hat{K}$ (which is comparable to the Euclidean metric) and let $\mu$ be the (normalized) Hausdorff measure on $\hat{K}$ with respect to $d$. Brownian motion has been constructed on $\hat{K}$ and it has been proved in $[\mathrm{BP}]$ that its heat kernel $\{q(t, x, y): t>0, x, y \in \hat{K}\}$ enjoys the following estimates for all $t>0, x, y \in \hat{K}$ :

$$
c_{1} t^{-d_{f} / d_{w}} \exp \left(-c_{2}\left(\frac{d(x, y)^{d_{w}}}{t}\right)^{\frac{1}{d_{w}-1}}\right) \leq q(t, x, y) \leq c_{3} t^{-d_{f} / d_{w}} \exp \left(-c_{4}\left(\frac{d(x, y)^{d_{w}}}{t}\right)^{\frac{1}{d_{w}-1}}\right),
$$


where $d_{f}=\log 3 / \log 2$ is the Hausdorff dimension and $d_{w}=\log 5 / \log 2$ is called the walk dimension.

We next consider a 2-dimensional Riemannian manifold (a fractal-like manifold) $M$, whose global structure is like that of the fractal. It can be constructed from $\hat{K}_{\text {pre }}$ by changing each bond to a cylinder and smoothing the connection to make it a manifold. One can naturally construct a Brownian motion on the surfaces of cylinders. Using the stability of sub-Gaussian heat kernel estimates (see for instance BBK1 for details), one can show that any divergence operator $\mathcal{L}=\sum_{i, j=1}^{2} \frac{\partial}{\partial x_{i}}\left(a_{i j}(x) \frac{\partial}{\partial x_{j}}\right)$ in local coordinates on such manifolds that satisfies the uniform elliptic condition obeys the following heat kernel estimates for all $t>0, x, y \in M$ :

$$
\begin{aligned}
\frac{c_{1}}{V\left(x, \Psi^{-1}(t)\right)} \exp \left(-c_{2}\left(\frac{\Psi(d(x, y))}{t}\right)^{\gamma_{1}}\right) & \leq q(t, x, y) \\
& \leq \frac{c_{3}}{V\left(x, \Psi^{-1}(t)\right)} \exp \left(-c_{4}\left(\frac{\Psi(d(x, y))}{t}\right)^{\gamma_{2}}\right)
\end{aligned}
$$

where $V(x, r) \asymp r^{2} \wedge r^{d_{f}}$ for all $x \in M, \Psi(s)=s^{2} \vee s^{d_{w}}$, and $\gamma_{1}, \gamma_{2}>0$ are some constants.

We now subordinate the diffusion $\left\{Z_{t}\right\}$ whose heat kernel enjoys (5.2). Let $\left\{\xi_{t}\right\}$ be a subordinator that is independent of $\left\{Z_{t}\right\}$; namely, it is an increasing Lévy process on $\mathbb{R}_{+}$. Let $\bar{\phi}$ be the Laplace exponent of the subordinator, i.e.

$$
\mathbb{E}\left[\exp \left(-\lambda \xi_{t}\right)\right]=\exp (-t \bar{\phi}(\lambda)), \quad \lambda, t>0 .
$$

In this example, for simplicity we consider the case $\bar{\phi}(t)=t^{\alpha_{1} / 2}+t^{\alpha_{2} / 2}$ for some $0<\alpha_{1} \leq$ $\alpha_{2}<2$, in which case, $\left\{\xi_{t}\right\}$ is a sum of independent $\alpha_{1} / 2$ - and $\alpha_{2} / 2$-subordinators. The process $\left\{X_{t}\right\}$ defined by $X_{t}=Z_{\xi_{t}}$ for any $t \geq 0$ is called a subordinate process. Define

$$
\phi(r)=\frac{1}{\bar{\phi}(1 / \Psi(r))} .
$$

It is easy to see $\phi$ satisfies (1.11). As discussed in [CKW1, Section 6.1], the heat kernel for $\left\{X_{t}\right\}$ satisfies $\operatorname{HK}(\phi)$ (hence $\operatorname{PHI}(\phi)$ as well) with

$$
\phi(r)=r^{\alpha_{2}} 1_{\{r \leq 1\}}+r^{\alpha_{1} d_{w} / 2} 1_{\{r>1\}},
$$

which is (up to constant multiplicative) the same as (5.3). Note that $\alpha_{1} d_{w} / 2>2$ when $\alpha_{1}$ is close to 2 .

It follows from our stability theorem for heat kernels, Theorem 1.11, that for any symmetric pure jump process on the above mentioned space whose jumping kernel enjoys $\mathrm{J}_{\phi}$ with $\phi$ given by (5.4), its heat kernel enjoys the estimates $\operatorname{HK}(\phi)$, hence $\operatorname{PHI}(\phi)$ holds for these processes.

The following example is taken from [CKi], which shows that PHI holds for the trace of Brownian motion on Sierpinski gasket on one side of the big triangle, by using the characterization of PHI from the main result of this paper, Theorem 1.20. 
Example 5.2. (Trace of Brownian motion on the Sierpinski gasket.) Let $K$ be the two-dimensional Sierpinski gasket obtained from the unit triangle with vertices $a_{1}=(0,0)$, $a_{2}=(1,0)$ and $a_{3}=(1 / 2, \sqrt{3} / 2)$ as in Example 5.1. It is known that there is a Brownian motion $X$ on $K$. Let $Y=\left\{Y_{t} ; t \geq 0\right\}$ be the trace process of $X$ on the line segment $I$ connecting $a_{1}$ and $a_{2}$. That is, $Y_{t}=X_{\tau_{t}}$ for $t \geq 0$, where $\tau_{t}=\inf \left\{s>0: A_{s}>t\right\}$ and $A_{t}$ is the positive continuous additive functional of $X$ whose Revuz measure $\mu$ is the onedimensional Lebesgue measure restricted to $I$. The trace process $Y$ is an $\mu$-symmetric pure jump process on $I$ with jumping measure $J(d x, d y)=J(x, y) \mu(d x) \mu(d y)$ (cf. [CF, [FOT]). For convenience, we identify the line segment $I$ with the unit interval $[0,1]$. Denote the Dirichlet form of $Y$ on $L^{2}(I ; \mu)$ by $(\mathcal{E}, \mathcal{F})$. Then the domain of the Dirichlet form $\mathcal{F}$ is the same as that of the symmetric $\alpha$-stable process on $I$, where $\alpha=\log (10 / 3) / \log 2 \in(1,2)$, and their corresponding $\mathcal{E}_{1}$-energies (i.e. $\mathcal{E}_{1}(u, u)$ ) are comparable. Kigami Kig computed the jumping kernel $J(x, y)$. From which, it is easy to deduce that there is a constant $c_{1}>0$ so that

$$
J(x, y) \leq c_{1}|x-y|^{-(1+\alpha)} \quad \text { for all } x, y \in[0,1] .
$$

However, $J(x, y)$ vanishes on some open subset of $[0,1]^{2}$ and is comparable to $|x-y|^{-(1+\alpha)}$ only on a proper subset $U$ of $[0,1]^{2}$. Let $g_{1}(x, y)=(x / 2, y / 2)$ and $g_{2}(x, y)=((1+x) / 2,(1+$ y)/2). Define

$$
D_{1}=\left\{(x, y) \in[0,1]^{2}:|x-y| \geq 1 / 2\right\}, \quad D_{k+1}=g_{1}\left(D_{k}\right) \cup g_{2}\left(D_{k}\right) \text { for } k \geq 1 \text { and } U=\cup_{k \geq 1} D_{k} .
$$

Then there is a constant $c_{2}>0$ so that

$$
J(x, y) \geq c_{2}|x-y|^{-(1+\alpha)} \quad \text { for all }(x, y) \in U .
$$

Define $\phi(r)=r^{\alpha}$. Then $\mathrm{J}_{\phi, \leq}$ holds for the trace process $Y$ in view of (5.5) .

For $x \in I=[0,1]$, define $U_{x}=\{y \in I:(x, y) \in U\}$. Then from the definition of $U$ in (5.6) (details are given in [CKi]), it is easy to see that there exist constants $c_{3}, c_{4} \in(0,1)$ such that for every $x \in I$ and $0<r \leq 1$,

$$
\mu\left(U_{x} \cap A\left(x, c_{3} r, r\right)\right) \geq c_{4} \mu\left(A\left(x, c_{3} r, r\right)\right) .
$$

Here for $0<r_{1}<r_{2}, A\left(x, r_{1}, r_{2}\right):=B\left(x, r_{2}\right) \backslash B\left(x, r_{1}\right)$. Thus by (5.7), for every $B_{r}=B(x, r)$ with $x \in I$ and $r \in(0,1]$ and every $f \in \mathcal{F}_{B_{r}}$,

$$
\begin{aligned}
\int_{B_{r}}\left(f-\bar{f}_{B_{r}}\right)^{2} d \mu & \leq C_{1} \phi(r) \int_{B_{r} \times B_{r}}(f(x)-f(y))^{2} \frac{1}{|x-y|^{1+\alpha}} d x d y \\
& \leq C_{2} \phi(r) \int_{B_{r} \times B_{r}}(f(x)-f(y))^{2} J(x, y) d x d y
\end{aligned}
$$

where the first inequality is due to the Poincaré inequality for symmetric $\alpha$-stable process on $I$, and the second inequality is due to (5.7), (5.8) and an argument similar to that of [BKS, Theorem 1.1]. Hence the finite range version of $\operatorname{PI}(\phi)$ holds for $Y$. By Remark 1.7, SCSJ $(\phi)$ and hence $\operatorname{CSJ}(\phi)$ automatically holds since $\alpha<2$. It is easy to see that UJS holds as well in view of (5.5) and (5.7). Therefore by Theorem [1.20 and Remark 1.22, the finite range 
version of $\mathrm{PHI}(\phi)$ holds for $Y$; that is, $\mathrm{PHI}(\phi)$ holds for non-negative caloric functions of $Y$ in any cylinder with $r \leq 1$.

Since the jumping kernel $J(x, y)$ vanishes on some open subsets of $[0,1]^{2}$, it does not satisfy $\mathrm{J}_{\phi}$ condition. Hence $Y$ does not have two-sided heat kernel estimates $\mathrm{HK}(\phi)$. However, by (5.5) and [CK2, the proof of Theorem 1.2 and Remark 4.4], we can show that the transition density function $p(t, x, y)$ of $Y$ with respect to the Lebesgue measure $\mu$ on $I$ has the following upper bound estimate: there is a constant $c_{5}>0$ so that

$$
p(t, x, y) \leq c_{5}\left(t^{-1 / d} \wedge \frac{t}{|x-y|^{1+\alpha}}\right) \quad \text { for all }(t, x, y) \in(0,1] \times I \times I .
$$

That is, $\operatorname{UHK}(\phi)$ holds for $Y$ over any bounded time interval. Although the corresponding lower bound estimate fails for $Y$, a main result of CKi] asserts that the corresponding lower bound holds for $Y$ over the subset $U$ of $I^{2}$; that is, there is a constant $c_{6}>0$ so that

$$
p(t, x, y) \geq c_{6}\left(t^{-1 / d} \wedge \frac{t}{|x-y|^{1+\alpha}}\right) \quad \text { for all }(t, x, y) \in(0,1] \times U .
$$

In the remainder of this section, we give some more details for Examples 1.2 1.3, and show that some conditions in the equivalence statements of Theorem 1.20 are necessary through two more examples.

Example 1.2 (continued): Here we provide some more details for this example. Clearly $\mathrm{J}_{\phi, \leq}$ and UJS hold. Since PI $(\phi)$ holds for rotationally symmetric stable process on $\mathbb{R}^{d}$ with $\phi(r)=r^{\alpha}$, it follows from [DK, Example 3] or [BKS, Theorem 1.1] that PI $(\phi)$ holds for the symmetric non-local Dirichlet form with jumping kernel $J(x, y)$. By Remark 1.7. SCSJ $(\phi)$ and hence $\operatorname{CSJ}(\phi)$ holds. So we have $\operatorname{PHI}(\phi)$ by Theorem 1.20 . However, since $\mathrm{J}_{\phi, \geq}$ does not hold, $\operatorname{HK}(\phi)$ does not hold either in view of Theorem 1.11.

Example 1.3 (continued): We now provide some more details for this example. Clearly $\mathrm{J}_{\phi, \leq}$ holds. We show below in Proposition 5.3 that UJS holds when the domain parameter $r_{\theta}$ in the increment condition of $\xi(x)$ is sufficiently small. On the other hand, by BKS, Theorem 1.1], there is a constant $C_{0}>0$ so that for every ball $B \subset \mathbb{R}^{d}$, every $f \in L^{2}(B ; d x)$ and every $\beta \in\left[\alpha_{1}, 2\right)$

$$
\int_{B \times B} \frac{(f(x)-f(y))^{2}}{|x-y|^{d+\beta}}\left(\mathbf{1}_{\Gamma_{\theta}(x)}(y)+\mathbf{1}_{\Gamma_{\theta}(y)}(x)\right) d x d y \geq C_{0} \int_{B \times B} \frac{(f(x)-f(y))^{2}}{|x-y|^{d+\beta}} d x d y .
$$

Integrating in $\beta$ with respect to the probability measure $\nu$ over $\left[\alpha_{1}, \alpha_{2}\right]$ yields

$$
\begin{aligned}
& \int_{B \times B}(f(x)-f(y))^{2} J(x, y) d x d y \\
& \geq C^{-1} \int_{\alpha_{1}}^{\alpha_{2}} \int_{B \times B} \frac{(f(x)-f(y))^{2}}{|x-y|^{d+\beta}}\left(\mathbf{1}_{\Gamma_{\theta}(x)}(y)+\mathbf{1}_{\Gamma_{\theta}(y)}(x)\right) d x d y \nu(d \beta) \\
& \geq C^{-1} C_{0} \int_{B \times B} \frac{(f(x)-f(y))^{2}}{|x-y|^{d} \phi(|x-y|)} d x d y
\end{aligned}
$$


where $C \geq 1$ is the constant in (1.6). The other direction of the inequality

$$
\int_{B \times B}(f(x)-f(y))^{2} J(x, y) d x d y \leq C \int_{B \times B} \frac{(f(x)-f(y))^{2}}{|x-y|^{d} \phi(|x-y|)} d x d y
$$

follows directly from (1.6). It has been established in [CK2 that the symmetric Markov process on $\mathbb{R}^{d}$ with jumping kernel $\frac{1}{|x-y|^{d} \phi(|x-y|)}$ has two-sided heat kernel estimates $\mathrm{HK}(\phi)$ and so $\operatorname{PI}(\phi)$ holds for this process in view of Corollary 1.21 and Theorem 1.20. Hence we deduce from the above inequalities that $\operatorname{PI}(\phi)$ holds for the symmetric non-local Dirichlet form with jumping kernel $J(x, y)$. By Remark 1.7. $\operatorname{SCSJ}(\phi)$ and hence $\operatorname{CSJ}(\phi)$ hold. Therefore PHI $(\phi)$ holds by Theorem 1.20. Moreover, by Theorem 1.20, $\mathrm{PHR}(\phi), \mathrm{EHR}$ as well as $\mathrm{E}_{\phi}$ hold.

Proposition 5.3. UJS holds for Example 1.3 when the parameter $r_{\theta}>0$ in (1.5) is sufficiently small.

Proof. Case $1, x \in \Xi(y)$ : In this case, either $x \in \Gamma_{\theta}(y)$ or $x \in \overline{B(y, 1)}$. In each case, it is easy to verify that for $0<r \leq d(x, y) / 2$,

$$
|\{z \in B(x, r): z \in \Xi(y)\}| \geq c_{\theta, 1} r^{2}
$$

with some $c_{\theta, 1}>0$, hence UJS holds.

Case 2, $y \in \Xi(x)$ and $d(x, y) \leq 1$ : In this case, $y \in \overline{B(x, 1)}$, and it is once again easy to verify that for $0<r \leq d(x, y) / 2$,

$$
|\{z \in B(x, r): y \in \Xi(z)\}| \geq c_{\theta, 2} r^{2}
$$

with some $c_{\theta, 2}>0$, hence UJS holds.

Case 3, $y \in \Xi(x)$ and $d(x, y)>1$ : In this case, $y \in \Gamma_{\theta}(x)$. We further divide it into two cases. Recall that $c_{\theta}>0$ is the constant so that $\xi\left(x+c_{\theta}\right)=\xi(x)+2 \pi$ for $x \in \mathbb{R}$.

i) When $r \in\left(0, c_{\theta}\right]$ : Let $s \in\left(0, r \wedge r_{\theta}\right]$, and $y^{\prime}$ be either $y-(s, 0)$ or $y+(s, 0)$. In each case the angle $\angle y x y^{\prime}$ is at most $\sin ^{-1} s$. Hence by assumption (1.5) and translation, we have either $y \in \Gamma_{\theta}(x+(s, 0))$ for all $s \leq r \wedge r_{\theta}$ or $y \in \Gamma_{\theta}(x-(s, 0))$ for all $s \leq r \wedge r_{\theta}$. Suppose the former holds. (One can argue similarly if the latter holds.) Then, because $v(x)$ depends only on the first coordinate of $x$, we have either $y \in \Gamma_{\theta}\left(x+\left(s, s^{\prime}\right)\right)$ for all $s^{\prime} \leq r \wedge r_{\theta}$ or $y \in \Gamma_{\theta}\left(x+\left(s,-s^{\prime}\right)\right)$ for all $s^{\prime} \leq r \wedge r_{\theta}$. Suppose the former holds. (Again, we can argue similarly if the latter holds.) Then we have

$$
y \in \Gamma_{\theta}(z) \quad \text { for all } z \in D:=\left\{x+\left(s, s^{\prime}\right): s, s^{\prime} \in\left(0, r \wedge r_{\theta}\right]\right\}
$$

Hence $|D| \asymp\left(r \wedge r_{\theta}\right)^{2}$ so that (5.9) holds, which implies UJS.

ii) When $r>c_{\theta}$ : Let

$$
H_{j}:=\left\{z=\left(z_{1}, z_{2}\right): z_{1}=x_{1}+j c_{\theta}, d(z, y) \geq d(x, y)\right\} \cap \Gamma_{\theta}\left(x ; y^{c}\right), \quad j \in \mathbb{Z},
$$

where $\Gamma_{\theta}\left(x ; y^{c}\right)$ is the connected component of $\Gamma_{\theta}(x) \backslash\{x\}$ that does not contain $y$. Depending on whether $\Gamma_{\theta}(x)$ contains $\left\{z=\left(z_{1}, z_{2}\right): z_{1}=x_{1}\right\}$ or not and depending on the angle of the cone, it holds that either the length of $H_{j} \cap B(x, r)$ is of order $\left(\left[r / c_{\theta}\right]-|j|\right)$ or $|j|$ for 
either $j=0,1, \cdots,\left[r / c_{\theta}\right]$ or $j=0,-1, \cdots,-\left[r / c_{\theta}\right]$. Among the four cases, let us discuss the first case (the other cases can be discussed similarly). Since $\Gamma_{\theta}(z)$ is a translation of $\Gamma_{\theta}(x)$ (because of the periodicity of $v(\cdot)$ ), it holds that $y \in \Gamma_{\theta}(z)$ for all $z \in H_{j}$. By the conclusion (5.10) of Case $3 \mathrm{i}$ ) with $r=c_{\theta}$, for any $j=0,1, \cdots,\left[r / c_{\theta}\right]$, there exists a rectangle $G_{j}$ with width $c_{\theta} \wedge r_{\theta}$ from Case $3 \mathrm{i}$ ) and having $H_{j} \cap B(x, r)$ as one of its vertical side such that $y \in \Gamma_{\theta}\left(z^{\prime}\right)$ for all $z^{\prime} \in G_{j}$. Hence, $\left|G_{j}\right| \geq\left(c_{\theta} \wedge r_{\theta}\right)\left(\left[r / c_{\theta}\right]-j\right)$, and so $\left|\cup_{j=1}^{\left[r / c_{\theta}\right]} G_{j}\right| \geq \sum_{j=1}^{\left[r / c_{\theta}\right]}\left(c_{\theta} \wedge r_{\theta}\right)\left(\left[r / c_{\theta}\right]-j\right) \geq c_{\theta, 3} r^{2}$ for some $c_{\theta, 3}>0$. Thus (5.9) holds with $c_{\theta, 2}>0$ being replaced by a different constant $c_{\theta, 4}>0$, which implies UJS.

Example 5.4. (EHI and $\mathrm{E}_{\phi}$ do not imply $\mathrm{PHI}(\phi)$.) Let $M=\mathbb{R}^{2}$ and $1<\alpha<2$. Consider a symmetric Lévy process $X=\left\{X_{t}\right\}$ on $\mathbb{R}^{2}$ with the Lévy measure of the form

$$
\nu(d x)=h(x) d x:=|x|^{-2-\alpha} f(x /|x|) d x,
$$

where $f: \mathcal{S}^{1} \rightarrow \mathbb{R}_{+}$is bounded and symmetric. Then, it is proved in [BS, Corollary 13] that EHI holds for non-negative harmonic functions. In fact, [BS, Theorem 1] gives more general fact in $\mathbb{R}^{d}$ setting with $d \geq 1$ that EHI holds for non-negative harmonic functions on $B(0,1)$ if and only if there is a constant $C>0$ such that the following holds

$$
\int_{B(y, 1 / 2)}|y-v|^{\alpha-d} h(v) d v \leq C \int_{B(y, 1 / 2)} h(v) d v, \quad|y|>1 .
$$

Let us take a particular choice of $f$ given as follows. For $i \in \mathbb{N}$, let $\theta_{i}=(3 \pi / 8) 4^{-i}$ and $\theta_{i}^{\prime}=(3 \pi / 8) 2^{-i}$. Note that $\sum_{i=1}^{\infty}\left(\theta_{i}+\theta_{i}^{\prime}\right)=\pi / 2$. Define

$$
H=\left\{e^{\theta \sqrt{-1}}, e^{-\theta \sqrt{-1}},-e^{\theta \sqrt{-1}},-e^{-\theta \sqrt{-1}}: \theta \in A\right\}
$$

where

$$
A=\left[0, \theta_{1}\right) \cup\left(\bigcup_{n=1}^{\infty}\left[\sum_{i=1}^{n}\left(\theta_{i}+\theta_{i}^{\prime}\right), \sum_{i=1}^{n}\left(\theta_{i}+\theta_{i}^{\prime}\right)+\theta_{n+1}\right)\right) .
$$

Set $f(x)=\mathbf{1}_{H}(x)$. Then, writing $\xi_{n}=\sum_{i=1}^{n}\left(\theta_{i}+\theta_{i}^{\prime}\right)+\theta_{n+1} / 2$ and $J(x, y)=h(x-y)$, we see that

$$
J\left(e^{\xi_{n} \sqrt{-1}}, 0\right)=1 \text {. }
$$

Setting $H_{n}=\left\{e^{\theta \sqrt{-1}}: \theta \in\left[\xi_{n}-\theta_{n+1} / 2, \xi_{n}+\theta_{n+1} / 2\right)\right\}$, we have for large $n$,

$$
\begin{aligned}
V\left(e^{\xi_{n} \sqrt{-1}}, 2^{-n-1}\right)^{-1} \int_{B\left(e^{\xi_{n} \sqrt{-1}}, 2^{-n-1}\right)} J(z, 0) d z & \leq c\left(2^{n+1}\right)^{2} \int_{B\left(e^{\xi_{n} \sqrt{-1}}, 2^{-n-1}\right)} \mathbf{1}_{H_{n}}(z /|z|) d z \\
& \leq c^{\prime} 4^{n} 2^{-n-1} 4^{-n-1} \leq c_{0} 2^{-n}
\end{aligned}
$$

so UJS does not hold. Therefore, by Theorem 1.20, PHI $(\phi)$ can not hold in this case.

We will briefly explain why $\mathrm{E}_{\phi}$ holds with $\phi(r)=r^{\alpha}$. Note that the corresponding generator can be written as follows

$$
\mathcal{L} u(x)=\int_{\mathbb{R}^{2}}\left(u(x+z)-u(x)-\nabla u(x) \cdot z \mathbf{1}_{\{|z|<1\}}\right) \nu(d z) .
$$


For $g \in C_{b}^{2}\left(\mathbb{R}^{2}\right)$ with $0 \leq g \leq 1$, let $g_{r}(y)=g(y / r)$ for $r>0$. Then, by similar computations as in [KSV, Lemma 13.4.1], we have $\left|\mathcal{L} g_{r}\right| \leq c_{1} r^{-\alpha}$, and so $\mathbb{P}^{0}\left(\tau_{B(0, r)} \leq t\right) \leq c_{2} t / r^{\alpha}$ for all $t, r>0$. This implies

$$
\mathbb{E}^{0}\left[\tau_{B(0, r)}\right] \geq \frac{r^{\alpha}}{2 c_{2}} \mathbb{P}^{0}\left(\tau_{B(0, r)} \geq r^{\alpha} /\left(2 c_{2}\right)\right) \geq \frac{r^{\alpha}}{4 c_{2}},
$$

so that (since the process is the Lévy process) $\mathrm{E}_{\phi, \geq}$ holds. Next we have by the Lévy system formula,

$$
\begin{aligned}
\mathbb{P}^{0}\left(\tau_{B(0, r)} \leq r^{\alpha}\right) & \geq \mathbb{P}^{0}\left(X \text { hits } B(0,6 r) \backslash B(0,3 r) \text { by time } r^{\alpha}\right) \\
& \geq \mathbb{P}^{0}\left(X_{r^{\alpha} \wedge \tau_{B(0, r)}} \in B(0,6 r) \backslash B(0,3 r)\right) \\
& =\mathbb{E}^{0}\left[\int_{0}^{r^{\alpha} \wedge \tau_{B(0, r)}} \nu\left((B(0,6 r) \backslash B(0,3 r))-X_{s}\right) d s\right] \\
& \geq \nu(B(0,5 r) \backslash B(0,4 r)) \mathbb{E}^{0}\left[r^{\alpha} \wedge \tau_{B(0, r)]}\right. \\
& \geq \frac{c_{3}}{r^{\alpha}} \mathbb{E}^{0}\left[r^{\alpha} \wedge \tau_{B(0, r)}\right] \\
& \geq \frac{c_{3}}{r^{\alpha}} \cdot \frac{r^{\alpha}}{2 c_{2}} \mathbb{P}^{0}\left(\tau_{B(0, r)} \geq r^{\alpha} /\left(2 c_{2}\right)\right) \geq \frac{c_{3}}{4 c_{2}}=: c_{4} .
\end{aligned}
$$

It follows that $\mathbb{P}^{0}\left(\tau_{B(0, r)}>r^{\alpha}\right) \leq 1-c_{4}$. Iterating this as in the proof of Proposition 3.5)(ii), we obtain $\mathrm{E}_{\phi, \leq}$.

Though the following example is not in the framework of our paper since the Lévy measure is singular to the Lebesgue measure on $\mathbb{R}^{d}$, it illustrates that in the context of symmetric jump processes, EHI in general does not follow from EHR and $\mathrm{E}_{\phi}$ alone.

Example 5.5. (EHR and $\mathrm{E}_{\phi}$ do not imply $\mathrm{EHI}$ nor $\mathrm{PHI}(\phi)$.) Let $M=\mathbb{R}^{3}$ and $0<$ $\alpha<2$. Consider a symmetric process $X_{t}=\left(X_{t}^{(1)}, X_{t}^{(2)}, X_{t}^{(3)}\right)$, where $X_{t}^{(i)}, i=1,2,3$, are independent 1-dimensional symmetric $\alpha$-stable processes. In [BC], it is proved that $X=\left\{X_{t} ; t \geq 0\right\}$ satisfies EHR and $\mathrm{E}_{\phi}$ with $\phi(r)=r^{\alpha}$, but EHI and, consequently $\operatorname{PHI}(\phi)$, fails too. In addition, in this case one can easily see that UJS does not hold. (We note that in $\left[\mathrm{BC}\right.$, the authors discussed more general processes on $\mathbb{R}^{d}$ that are expressed by a system of stochastic differential equations $d X_{t}=A\left(X_{t-}\right) d Z_{t}$, where $Z_{t}^{(i)}, 1 \leq i \leq d$, are independent 1-dimensional symmetric $\alpha$-stable processes and $A$ is a matrix-valued function which is bounded, continuous and non-degenerate.) We also note that for this example, $\operatorname{PI}(\phi)$ and $\operatorname{SCSJ}(\phi)$ are satisfied by [DK, Example 4] and Remark 1.7, respectively.

Acknowledgements. We thank Dr. M. Murugan for spotting the inconsistent use of time direction in Definition 1.15(iii) of an earlier version of this paper. We also thank Prof. M. Kassmann and Dr. T. Schulze for pointing out an error in Example 1.3 of an earlier version of this paper. We are grateful to the referees for reading the paper carefully and helpful comments. 


\section{References}

[BBCK] M.T. Barlow, R.F. Bass, Z.-Q. Chen and M. Kassmann. Non-local Dirichlet forms and symmetric jump processes. Trans. Amer. Math. Soc. 361 (2009), 1963-1999.

[BBK1] M.T. Barlow, R.F. Bass and T. Kumagai. Stability of parabolic Harnack inequalities on metric measure spaces. J. Math. Soc. Japan 58 (2006), 485-519.

[BBK2] M.T. Barlow, R.F. Bass and T. Kumagai. Parabolic Harnack inequality and heat kernel estimates for random walks with long range jumps. Math. Z. 261 (2009), 297-320.

[BGK] M.T. Barlow, A. Grigor'yan and T. Kumagai. On the equivalence of parabolic Harnack inequalities and heat kernel estimates. J. Math. Soc. Japan 64 (2012), 1091-1146.

[BP] M.T. Barlow and E.A. Perkins. Brownian motion on the Sierpiński gasket, Probab. Theory Relat. Fields 79 (1988), 543-623.

[BC] R.F. Bass and Z.-Q. Chen. Regularity of harmonic functions for a class of singular stable-like processes. Math. Z. 266 (2010), 489-503.

[BL1] R.F. Bass and D. Levin. Harnack inequalities for jump processes. Potential Anal. 17 (2002), $375-388$.

[BL2] R.F. Bass and D. Levin. Transition probabilities for symmetric jump processes. Trans. Amer. Math. Soc. 354 (2002), 2933-2953.

[BS] K. Bogdan and P. Sztonyk. Harnack's inequality for stable Lévy processes. Potential Anal. 22 (2005), 133-150.

[BKS] K.-U. Bux, M. Kassmann and T. Schulze. Quadratic forms and Sobolev spaces of fractional order. Proc. London Math. Soc. 119 (2019), 841-866.

[CS] L. Caffarelli and L. Silvestre. Regularity theory for fully nonlinear integro-differential equations. Comm. Pure Appl. Math. 62 (2009), 597-638.

[CKP1] A.D. Castro, T. Kuusi and G. Palatucci. Local behavior of fractional p-minimizers. Ann. Inst. H. Poincaré Anal. Non Linéaire 33 (2016), 1279-1299.

[CKP2] A.D. Castro, T. Kuusi and G. Palatucci. Nonlocal Harnack inequalities. J. Funct. Anal. 267 (2014), 1807-1836.

[C] Z.-Q. Chen. On notions of harmonicity. Proc. Amer. Math. Soc. 137 (2009), 3497-3510.

[CF] Z.-Q. Chen and M. Fukushima. Symmetric Markov Processes, Time Change, and Boundary Theory. Princeton Univ. Press, Princeton 2012.

[CKi] Z.-Q. Chen and J. Kigami. Observable heat kernel estimate. In preparation.

[CKK1] Z.-Q. Chen, P. Kim and T. Kumagai. On heat kernel estimates and parabolic Harnack inequality for jump processes on metric measure spaces. Acta Math. Sin. (Engl. Ser.) 25 (2009), 1067-1086. 
[CKK2] Z.-Q. Chen, P. Kim and T. Kumagai. Global heat kernel estimates for symmetric jump processes. Trans. Amer. Math. Soc. 363 (2011), 5021-5055.

[CK1] Z.-Q. Chen and T. Kumagai. Heat kernel estimates for stable-like processes on $d$-sets. Stochastic Process Appl. 108 (2003), 27-62.

[CK2] Z.-Q. Chen and T. Kumagai. Heat kernel estimates for jump processes of mixed types on metric measure spaces. Probab. Theory Relat. Fields 140 (2008), 277-317.

[CKW1] Z.-Q. Chen, T. Kumagai and J. Wang. Stability of heat kernel estimates for symmetric non-local Dirichlet forms. To appear in Memoirs Amer. Math. Soc.

[CKW3] Z.-Q. Chen, T. Kumagai and J. Wang. Elliptic Harnack inequalities for symmetric nonlocal Dirichlet forms. J. Math. Pures et Appliquées 125 (2019), 1-42.

[ChK] Z.-Q. Chen and K. Kuwae. On subhamonicity for symmetric Markov processes. J. Math. Soc. Japan 64 (2012), 1181-1209.

[ChZ] Z.-Q. Chen and X. Zhang. Hölder estimates for nonlocal-diffusion equations with drifts. Commun. Math. Stat. 2 (2014), 331-348.

[De] T. Delmotte. Parabolic Harnack inequality and estimates of Markov chains on graphs. Rev. Mat. Iberoamericana 15 (1999), 181-232.

[DK] B. Dyda and M. Kassmann. Regularity estimates for elliptic nonlocal operators. Analysis \& PDE 13 (2020), 317-370.

[FOT] M. Fukushima, Y. Oshima and M. Takeda. Dirichlet Forms and Symmetric Markov Processes. de Gruyter, Berlin, 2nd rev. and ext. ed., 2011.

[G] E. Giusti. Direct Methods in the Calculus of Variations. World Scientific Publishing Co. Inc., River Edge 2003.

[Gr] A. Grigor'yan. The heat equation on noncompact Riemannian manifolds. (in Russian) Matem. Sbornik. 182 (1991), 55-87. (English transl.) Math. USSR Sbornik 72 (1992), 47-77.

[GHH] A. Grigor'yan, E. Hu and J. Hu. Two-sided estimates of heat kernels of jump type Dirichlet forms. Adv. Math. 330 (2018), 433-515.

[GH] A. Grigor'yan and J. Hu. Upper bounds of heat kernels on doubling spaces. Mosco Math. J. 14 (2014), 505-563.

[GT] A. Grigor'yan and A. Telcs. Two-sided estimates of heat kernels on metric measure spaces. Ann. Probab. 40 (2012), 1212-1284.

[HS] W. Hebisch and L. Saloff-Coste. On the relation between elliptic and parabolic Harnack inequalities. Ann. Inst. Fourier 51 (2001), 1437-1481.

[H] J. Heinonen. Lectures on Analysis on Metric Spaces. Springer-Verlag, New York 2001.

[K1] M. Kassmann. Harnack inequalities: An introduction. Boundary Value Problems 2007 (2007), Article ID 81415, 21 pages. 
[K2] M. Kassmann. A priori estimates for integro-differential operators with measurable kernels. Calc. Var. Partial Differ. Equ. 34 (2009), 1-21.

[Kig] J. Kigami. Personal communication.

[KSV] P. Kim, R. Song and Z. Vondraček. Potential theory of subordinate Brownian motions revisited. Stochastic Analysis and Applications to Finance - Essays in Honour of Jia-an Yan. World Scientific, 2012, pp. 243-290.

[Kom] T. Komatsu. Uniform estimates for fundamental solutions associated with non-local Dirichlet forms. Osaka J. Math. 32 (1995), 833-860.

[KS] S. Kusuoka and D. Stroock. Applications of the Malliavin calculus. III, J. Fac. Sci. Univ. Tokyo Sect. IA Math. 34 (1987), 391-442.

[KZ] S. Kusuoka and X.Y. Zhou. Dirichlet forms on fractals: Poincaré constant and resistance. Probab. Theory Relat. Fields 93 (1992), 169-196.

[LS] J. Lierl. Parabolic Harnack inequality for time-dependent non-symmetric Dirichlet forms. To appear in J. Math. Pures et Appliquées

[MK] A. Mimica and M. Kassmann. Intrinsic scaling properties for nonlocal operators. J. Eur. Math. Soc. 19 (2017), 983-1011.

[MS] M. Murugan and L. Saloff-Coste. Heat kernel estimates for anomalous heavy-tailed random walks. Ann. Inst. Henri Poincaré Probab. Stat. 55 (2019), 697-719.

[Sa1] L. Saloff-Coste. A note on Poincaré, Sobolev, and Harnack inequalities. Inter. Math. Res. Notices 2 (1992), 27-38.

[Sa2] L. Saloff-Coste. Aspects of Sobolev-type Inequalities. Lond. Math. Soc. Lect. Notes, vol. 289, Cambridge University Press, Cambridge 2002.

[SU] R. Schilling and T. Uemura. On the Feller property of Dirichlet forms generated by pseudo differential operators. Tohoku Math. J. 59 (2007), 401-422.

[Si] M.L. Silverstein. Symmetric Markov Processes. Lecture Notes in Mathematics, vol. 426, Springer-Verlag, Berlin Heidelberg 1974.

[Sil] L. Silvestre. Hölder estimates for solutions of integro-differential equations like the fractional Laplace. Indiana Univ. Math. J. 55 (2006), 1155-1174.

[SV] R. Song and Z. Vondraček. Harnack inequality for some classes of Markov processes. Math. Z. 246 (2004), 177-202.

[St] K.-T. Sturm. Analysis on local Dirichlet spaces III. The parabolic Harnack inequality. J. Math. Pures Appl. 75 (1996), 273-297.

\section{Zhen-Qing Chen}

Department of Mathematics, University of Washington, Seattle, WA 98195, USA 
E-mail: zqchen@uw.edu

\section{Takashi Kumagai:}

Research Institute for Mathematical Sciences, Kyoto University, Kyoto 606-8502, Japan

Email: kumagai@kurims.kyoto-u.ac.jp

\section{Jian Wang:}

College of Mathematics and Informatics \& Fujian Key Laboratory of Mathematical Analysis and Applications (FJKLMAA) \& Center for Applied Mathematics of Fujian Province (FJNU), Fujian Normal University, 350007 Fuzhou, P.R. China.

Email: jianwang@f jnu.edu.cn 